\title{
The Curvelet Representation of Wave Propagators is Optimally Sparse
}

\author{
Emmanuel J. Candès and Laurent Demanet \\ Applied and Computational Mathematics \\ California Institute of Technology \\ Pasadena, California 91125
}

\begin{abstract}
This paper argues that curvelets provide a powerful tool for representing very general linear symmetric systems of hyperbolic differential equations. Curvelets are a recently developed multiscale system $[9,5]$ in which the elements are highly anisotropic at fine scales, with effective support shaped according to the parabolic scaling principle width $\approx$ length $h^{2}$ at fine scales. We prove that for a wide class of linear hyperbolic differential equations, the curvelet representation of the solution operator is both optimally sparse and well organized.

- It is sparse in the sense that the matrix entries decay nearly exponentially fast (i.e. faster than any negative polynomial),

- and well-organized in the sense that the very few nonnegligible entries occur near a shifted diagonal.

Indeed, we actually show that the action of the wave-group on a curvelet is wellapproximated by simply translating the center of the curvelet along the Hamiltonian flow - hence the diagonal shift in the curvelet representation. A physical interpretation of this result is that curvelets may be viewed as coherent waveforms with enough frequency localization so that they behave like waves but at the same time, with enough spatial localization so that they simultaneously behave like particles.
\end{abstract}

Keywords. Hyperbolic Equations, Waves, Hamiltonian Equations, Characteristics, Geometrical Optics, Fourier Integral Operators, Curvelets, Sparsity, Nonlinear Approximation, Multiscale Representations, Parabolic Scaling.

Acknowledgments. This research was supported by a National Science Foundation grant DMS 01-40698 (FRG), by a DOE grant DE-FG03-02ER25529, and by an Alfred P. Sloan Fellowship. E. C. would like to thank Guillaume Bal for valuable conversations. 


\section{Introduction}

This paper is concerned with the representation of symmetric systems of linear hyperbolic differential equations of the form

$$
\frac{\partial u}{\partial t}+\sum_{k} A_{k}(x) \frac{\partial u}{\partial x_{k}}+B(x) u=0, \quad u(0, x)=u_{0}(x),
$$

where $u$ is an $m$-dimensional vector and $x \in \mathbb{R}^{n}$. The matrices $A_{k}$ and $B$ may depend on the spatial variable $x$, and the $A_{k}$ 's are symmetric. Linear hyperbolic systems are ubiquitous in the sciences and a classical example are the equations for acoustic waves which read

$$
\begin{aligned}
& \frac{\partial \rho}{\partial t}+\nabla \cdot\left(\rho_{0} u\right)=0 \\
& \rho_{0} \frac{\partial u}{\partial t}+\nabla\left(c_{0}^{2} \rho\right)=0,
\end{aligned}
$$

where $u$ and $\rho$ are the velocity and density disturbances respectively. (Here, $\rho_{0}=\rho_{0}(x)$ is the density and $c_{0}=c_{0}(x)$ is the speed of sound.) Other well-known examples include Maxwell's equations of electrodynamics and the equations of linear elasticity. All the results presented in this paper equally apply to higher-order scalar wave equations, e.g., of the form

$$
\frac{\partial^{2} u}{\partial t^{2}}-\sum_{i j} a_{i j}(x) \frac{\partial^{2} u}{\partial x_{j} \partial x_{k}}=0, \quad u(0, x)=u_{0}(x), \quad \frac{\partial u}{\partial t}(0, x)=u_{1}(x),
$$

(where $u$ is now a scalar and $a_{i j}(x)$ is taken to be symmetric and positive definite) as it is well-known that such single second-order equations can be reduced to a symmetric system of first-order equations (1.1) by appropriate changes of variables.

\section{$1.1 \quad$ About representations}

We are interested in representations of the solution operator $E(t)$ to the system (1.1)

$$
u(t, \cdot)=E(t) u_{0}
$$

which may be expressed as an integral involving the so-called Green's function

$$
u(t, x)=\int E(t ; x, y) u_{0}(y) d y .
$$

To introduce the concept of representation, suppose that the coefficient matrices do not depend on $x$. As is well-known, the Fourier transform is a powerful tool for studying differential equations in this setting. Indeed, in the Fourier domain, (1.1) takes the form

$$
\left(\partial_{t}+i \sum_{k} A_{k} \xi_{k}+B\right) \hat{u}(t, \xi)=0
$$

in short, (1.1) reduces to a system of ordinary differential equations which can be solved analytically. This shows the power of the representation; in the frequency domain, the solution operator is diagonal and the study becomes ridiculously simple.

These desirable properties are very fragile, however. Both mathematicians and computational scientists know that Fourier methods are not really amenable to differential equations with variable coefficients, and that we need to find alternatives. Instead of considering the evolution of Fourier coefficients, we may want to think, instead, of the action 
of the propagation operator $E(t)$ on other types of basis elements. This connects with the viewpoint of modern harmonic analysis whose goal is to develop representations, e.g. an orthonormal basis $\left(f_{n}\right)$ of $L^{2}\left(\mathbb{R}^{n}\right)$, say, in which the solution operator

$$
E\left(t ; n, n^{\prime}\right)=\left\langle f_{n}, E(t) f_{n^{\prime}}\right\rangle
$$

is as simple as possible; that is, such that $E(t) f_{n}$ is a sparse superposition of those elements $f_{n^{\prime}}$. Such sparse representations are extremely significant both in mathematical analysis, where sparsity allows for sharper inequalities and in numerical applications where sparsity allows for faster algorithms.

- In the field of mathematical analysis, for example, Calderón introduced what one would nowadays call the Continuous Wavelet Transform (CWT) in which objects are represented as a superpositions of simple elements of the form $\psi((x-b) / a)$, with $a>0$ and $b \in \mathbb{R}^{n}$; i.e, objects are represented as a superposition of dilates and translates of a single function $\psi$. These elements proved to be almost eigenfunctions of large classes of operators, the Calderón-Zygmund operators which are special types of singular integrals, some of which arising in connection with elliptic problems. It was later gradually realized that tools like atomic decompositions of Hardy spaces $[17,29]$ and orthonormal bases of Wavelets $[22,23]$ provide a setting in which some aspects of the mathematical analysis of these operators is dramatically eased.

- Clever representation of scientific and engineering computations can make previously intractable computations tractable. Here, sparsity may allow the design of fast matrix multiplication and/or fast matrix inversion algorithms. For example, Beylkin, Coifman and Rokhlin [2] exploited the sparsity of those singular integrals mentioned above, and showed how to use wavelet bases to compute such integrals with very low complexity algorithms.

In short, a single representation, namely, the wavelet transform provides sparse decompositions of large classes of operators simultaneously.

\subsection{Limitations of Classical Multiscale Ideas}

Our goal in this paper is to find a representation which provides sparse representations of the solution operators to fairly general classes of systems of hyperbolic differential equations. Now the last two decades have seen the widespread development of multiscale ideas such as Multigrid, Fast Multipole Methods, Wavelets, Finite Elements with or without adaptive refinement, etc. All these representations propose dictionaries of roughly isotropic elements occurring at all scales and locations; the templates are rescaled treating all directions in essentially the same way. Isotropic scaling may be successful when the object under study does not exhibit any special features along selected orientations. This is the exception rather than the rule.

Tools from traditional multiscale analysis are very powerful for representing certain elliptic problems but unfortunately, they are definitely ill-adapted to hyperbolic problems such as (1.1). Indeed,

1. they fail to sparsify the wave propagation, i.e. the solution operator $E(t)$,

2. and they fail to provide a sparse representation of oscillatory signals which are the solutions of those equations. 
To make things concrete, consider the problem of propagating elastic waves as in geophysics. Consider the scalar wave equation in two dimensions

$$
\partial_{t t} u=c^{2}(x) \Delta u,
$$

where $\Delta$ is the Laplacian defined by $\Delta=\partial^{2} / \partial x_{1}^{2}+\partial^{2} / \partial x_{2}^{2}$ (we may take the velocity field to be constant). To describe the action of the wave group, we assume that the initial condition takes the form of a wavelet with vanishing initial velocity, say. Then it is clear that at a later time, the wavefield is composed of large concentric rings (imagine throwing a stone in a lake). Now, it is also clear that many wavelets are needed to represent the wavefield. In other words, the wavefield is a rather dense superposition of wavelets. Note that this may be quantified. Suppose that the velocity field is identically equal to one, say, and that the initial condition is a wavelet at scale $2^{-j}$; that is, of the form $2^{j / 2} \psi\left(2^{j} x\right)$ so that in frequency, the energy is concentrated near the dyadic annulus $|\xi| \sim 2^{j}$. Then one would need at least $O\left(2^{j}\right)$ wavelets to reconstruct the wavefield at time $t=1$ to within reasonable accuracy.

Our simple example above shows wave-like flows do not preserve the geometry and characteristics of classical multiscale systems. To achieve sparsity, we need to rethink the geometry of multiscale representations.

\subsection{A New Form of Multiscale Analysis}

As we will see in section 2 , curvelets are waveforms which are highly anisotropic at fine scales, with effective support obeying the parabolic principle length $\approx$ widt $h^{2}$. Just as for wavelets, there is both a continuous and a discrete curvelet transform. A curvelet is indexed by three parameters which - adopting a continuous description of the parameter space - are: a scale $a, 0<a<1$; an orientation $\theta, \theta \in[-\pi / 2, \pi / 2)$ and a location $b, b \in \mathbb{R}^{2}$. At scale $a$, the family of curvelets is generated by translation and rotation of a basic element $\varphi_{a}$

$$
\varphi_{a, b, \theta}(x)=\varphi_{a}\left(R_{\theta}(x-b)\right) .
$$

Here, $\varphi_{a}(x)$ is some kind of directional wavelet with spatial width $\sim a$ and spatial length $\sim \sqrt{a}$, and with minor axis pointing in the horizontal direction

$$
\varphi_{a}(x) \approx \varphi\left(D_{a} x\right), \quad D_{a}=\left(\begin{array}{cc}
1 / a & 0 \\
0 & 1 / \sqrt{a}
\end{array}\right)
$$

$D_{a}$ is a parabolic scaling matrix, $R_{\theta}$ is a rotation by $\theta$ radians.

An important property is that curvelets obey the principle of harmonic of analysis which says that it is possible to analyze and reconstruct an arbitrary function $f\left(x_{1}, x_{2}\right)$ as a superposition of such templates. It is possible to construct tight-frames of curvelets and one can, indeed, easily expand an arbitrary function $f\left(x_{1}, x_{2}\right)$ as a series of curvelets, much like in an orthonormal basis. Continuing at an informal level of exposition, there is a sampling of the plane $(a, b, \theta)$

$$
a_{j}=2^{-j}, \quad \theta_{j, \ell}=2 \pi \ell \cdot 2^{-\lfloor j / 2\rfloor}, \quad R_{\theta_{j, \ell}} b_{k}^{(j, \ell)}=\left(k_{1} 2^{-j}, k_{2} 2^{-j / 2}\right),
$$

such that with $\mu$ indexing the triples $\left(a_{j}, \theta_{j, \ell}, b_{k}^{(j, \ell)}\right)$ the collection $\varphi_{\mu}$ is a tight-frame:

$$
f=\sum_{\mu}\left\langle f, \varphi_{\mu}\right\rangle \varphi_{\mu}, \quad\|f\|_{2}^{2}=\sum_{\mu}\left|\left\langle f, \varphi_{\mu}\right\rangle\right|^{2} .
$$




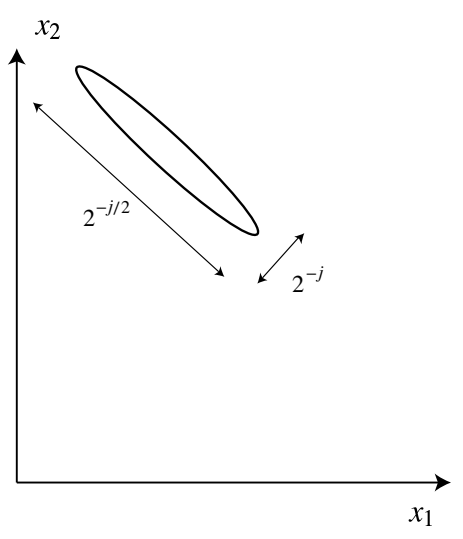

Spatial viewpoint

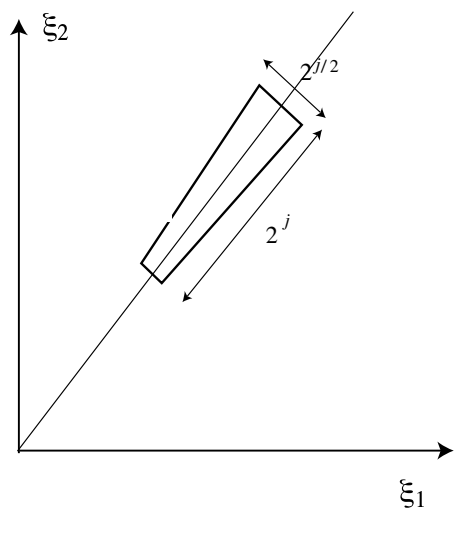

Frequency viewpoint

Figure 1: Schematic representation of the support of a curvelet in both space and frequency. In the spatial domain, a curvelet has an envelope strongly aligned along a specified 'ridge' while in the frequency domain, it is supported near a box whose orientation is aligned with the co-direction of the ridge.

(Note that these formulae allow us to analyze and synthesize arbitrary functions in $L^{2}\left(\mathbb{R}^{2}\right)$ as a superposition of curvelets in a stable and concrete way.)

As we have seen, a curvelet is well-localized in space but it is also well-localized in frequency. Recall that a given scale, curvelets $\varphi_{\mu}$ are obtained by applying shifts and rotations to a 'mother' curvelet $\varphi_{j, 0,0}$. In the frequency domain then

$$
\hat{\varphi}_{j, 0,0}(\xi)=2^{-3 j / 4} W\left(2^{-j}|\xi|\right) V\left(2^{\lfloor j / 2\rfloor} \theta\right) .
$$

Here, $W, V$ are smooth windows compactly supported near the intervals $[1,2]$ and $[-1 / 2,1 / 2]$ respectively. Whereas in the spatial domain curvelets live near an oriented rectangle $R$ of length $2^{-j / 2}$ and width $2^{-j}$, in the frequency domain, they are located in a parabolic wedge of length $2^{j}$ and width $2^{j / 2}$ and whose orientation is orthogonal to that of $R$. The joint localization in both space allows us to think about curvelets as occupying a 'Heisenberg cell' in phase-space with parabolic scaling in both domains. Figure 1 offers a schematic representation of this joint localization. As we shall see, this microlocal behavior is key to understanding the properties of curvelet-propagation. Additional details are given in section 2 .

\subsection{Curvelets and Geometrical Optics}

A hyperbolic system can typically be considered in the approximation of high-frequency waves, also known as geometrical optics. In order to best describe our main result, it is perhaps suitable first to exhibit the connections between curvelets and geometrical optics. In that setting it is not necessary to describe the dynamics in terms of the wavefield $u(t, x)$. Only its prominent features are studied: wave fronts, or equivalently rays. The latter are 
trajectories $(x(t), \xi(t))$ in phase-space $\mathbb{R}^{2} \times \mathbb{R}^{2}$, and are the solutions to the $m$ Hamiltonian flows (indexed by $\nu$ )

$$
\left\{\begin{aligned}
\dot{x}(t) & =\nabla_{\xi} \lambda_{\nu}^{0}(x, \xi), & & x(0)=x_{0}, \\
\dot{\xi}(t) & =-\nabla_{x} \lambda_{\nu}^{0}(x, \xi), & & \xi(0)=\xi_{0} .
\end{aligned}\right.
$$

The system (1.6) is also called the bicharacteristic flow and the rays $(x(t), \xi(t))$ the bicharacteristics. To see how this system arises, consider the classical high-frequency wavepropagation approximation to the wavefield $u(x, t)$ of the form

$$
u(x, t)=\sum_{\nu} e^{i \omega \Phi_{\nu}(x, t)}\left(\sigma_{\nu, 0}(x, t)+\frac{\sigma_{\nu, 1}(x, t)}{\omega}+\frac{\sigma_{\nu, 2}(x, t)}{\omega^{2}}+\ldots\right),
$$

where $\omega$ is a large parameter; it then follows (after substituting the approximation in the wave equation (1.1)) that the phase functions $\Phi_{\nu}$ must obey the eikonal equations

$$
\partial_{t} \Phi_{\nu}+\lambda_{\nu}^{0}\left(x, \nabla_{x} \Phi\right)=0
$$

and that the amplitudes must obey 'transport' equations we shall not detail here (see section 4). In the above expression (and in the Hamiltonian equations $(1.6)$ ), the $\lambda_{\nu}^{0}(x, \xi)$ are the eigenvalues of the dispersion matrix

$$
a^{0}(x, \xi)=\sum_{k} A_{k}(x) \xi_{j}
$$

It is well-known that the Hamiltonian equations describe the evolution of the wavefront set of the solution as $\Phi_{\nu}(t, x(t))$ is actually constant along the $\nu$ th Hamiltonian flow (1.6).

With this background, we are now in a position to qualitatively describe the behavior of the wave-propagation operator $E(t)$ acting on a curvelet $\varphi_{\mu}$. However, we first need to introduce a notion of vector-valued curvelet since $E(t)$ is acting on vector fields. Let $r_{\nu}^{0}(x, \xi)$ be the eigenvector of the dispersion matrix associated with the eigenvalue $\lambda_{\nu}^{0}(x, \xi)$. We then define hyper-curvelets by

$$
\varphi_{\mu \nu}^{(0)}(x)=\frac{1}{(2 \pi)^{2}} \int e^{i x \cdot \xi} r_{\nu}^{0}(x, \xi) \hat{\varphi}_{\mu}(\xi) d \xi
$$

Later in this section, we will motivate this special choice but for now simply observe that $\varphi_{\mu \nu}^{(0)}$ is a vector-valued waveform.

Consider then the solution to the wave equation $\varphi_{\mu \nu}^{(0)}(t, x)$ with initial value $\varphi_{\mu \nu}^{(0)}(x)$. Then we claim that

the action of the wave group on a hyper-curvelet is well-approximated by simply translating the center of the curvelet along the corresponding Hamiltonian flow.

To examine this claim, set $n(t)=\xi(t) /|\xi(t)|$ and consider the reduced Hamiltonian flow obtained by adding the equation

$$
\dot{U}(t)=-U\left(n \otimes \nabla_{x} \lambda_{\nu}^{0}(x, n)-\lambda_{\nu}^{0}(x, n) \otimes n\right)
$$

to the system (1.6). Here, $U(t)$ is the rotation matrix tracking the orientation rotation of $\xi(t)$ in the sense that at all times $U(t) n(t)=n(0)=\xi(0) /|\xi(0)|$, or $n(t)=U(t)^{*} n(0)$. 


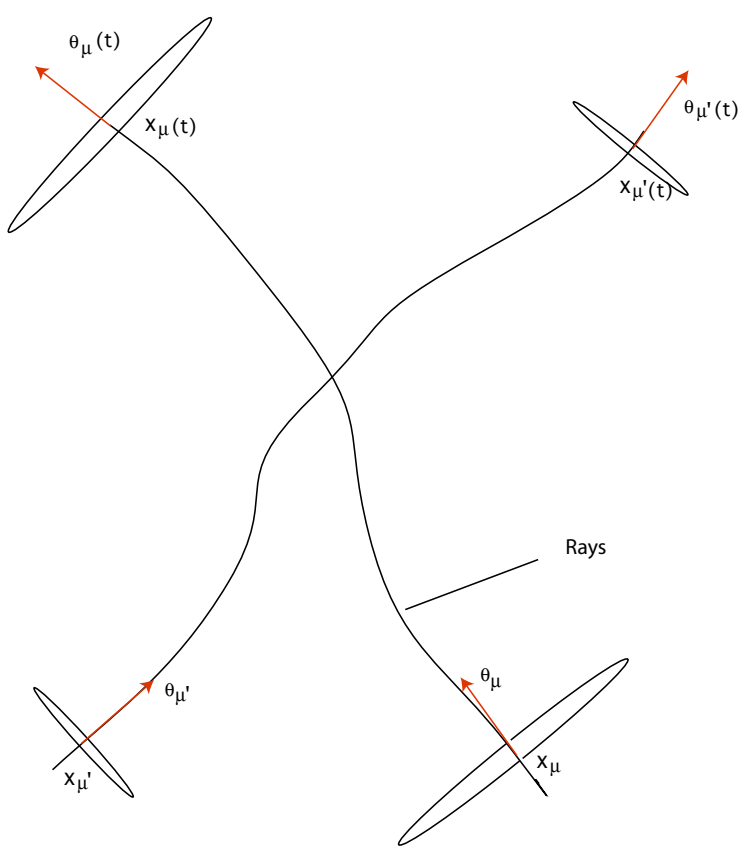

Figure 2: Schematic representation of the action of the wave group on a hyper-curvelet. The solution is well-approximated by rigid motion along the Hamiltonian flow.

Our claim says that the solution to the wave equation nearly follows the dynamics of the reduced Hamiltonian flow, i.e.

$$
\varphi_{\mu \nu}^{(0)}(t, x) \approx \varphi_{\mu \nu}^{(0)}\left(U_{\mu}(t)\left(x-x_{\mu}(t)\right)+x_{\mu}\right)
$$

where $x_{\mu}(t)$ and $U_{\mu}(t)$ are the rays of (1.10) with initial starting points $x_{\mu}(0)=x_{\mu}$ and $U_{\mu}(0)=I d$. Figure 2 illustrates this fact.

We now return to the interpretation of a hyper-curvelet. Suppose that $r_{\nu}^{0}$ only depends on $\xi$ as in the case of the acoustic system (1.2)

$$
r_{0}^{0}(\xi)=\left(\begin{array}{c}
\xi^{\perp} /|\xi| \\
0
\end{array}\right), \quad r_{ \pm}^{0}(\xi)=\frac{1}{\sqrt{2}}\left(\begin{array}{c} 
\pm \xi /|\xi| \\
1
\end{array}\right)
$$

(Here and below, $\xi^{\perp}$ denotes the vector obtained from $\xi$ after applying a rotation by 90 degrees). In this special case, we see that the hyper-curvelet is obtained by multiplyingin the frequency domain - a scalar-valued curvelet with the eigenvectors of the dispersion matrix

$$
\hat{\boldsymbol{\varphi}}_{\mu \nu}^{(0)}(\xi)=r_{\nu}^{0}(\xi) \hat{\varphi}_{\mu}(\xi), \quad \nu \in\{+,-, 0\} .
$$

This is useful for the curvelet $\hat{\varphi}_{\mu \nu}^{(0)}$ will essentially follow only one flow, namely, the $\nu$ th flow. Suppose we had started, instead, with an initial value of the form $\varphi_{\mu \nu}=\varphi_{\mu} \mathbf{e}_{\nu}$, where $\mathbf{e}_{\nu}$ is the canonical basis of $\mathbb{R}^{3}$, say. Then our curvelet would have interacted with the three eigenvectors of the dispersion matrix, and would have 'split' and followed the three distinct flows. By forcing $\hat{\boldsymbol{\varphi}}_{\mu \nu}^{(0)}(\xi)$ to be aligned with $r_{\nu}^{0}(\xi)$, we essentially removed the components associated with the other flows. In the general case (1.9), we build hyper-curvelets by applying $R_{\nu}^{0}$, which is now a pseudo-differential operator with symbol $r_{\nu}^{0}(x, \xi)$, mapping scalars to $m$-dimensional vectors, and independent of time. The effect is of course the same. 
Note that when $r_{\nu}^{0}$ is independent of $x$, hyper-curvelets build-up a (vector-valued) tightframe; letting $[F, G]$ be the usual inner product over three-dimensional vector fields in $L^{2}\left(\mathbb{R}^{2}\right)$, the family $\left(\varphi_{\mu \nu}^{(0)}\right)_{\mu \nu}$ obeys the reconstruction formula

$$
u=\sum_{\mu, \nu}\left[u, \varphi_{\mu \nu}^{(0)}\right] \varphi_{\mu \nu}^{(0)}
$$

and the Parseval relation

$$
\|u\|_{L^{2}}^{2}=\sum_{\mu, \nu}\left|\left[u, \varphi_{\mu \nu}^{(0)}\right]\right|^{2}
$$

Just as one can decompose a scalar field as a superposition of scalar curvelets, one can analyze and synthesize any wavefield as a superposition of hyper-curvelets in a stable and concrete way. For arbitrary $r_{\nu}^{0}(x, \xi)$, this is, however, in general not true.

We would like to emphasize that although the Eikonal equations only have solutions for small times, the approximation (1.11) and, more generally, all of our results are valid for all times since the rays (1.6) are always well-defined, see section 1.6 below for a more detailed discussion.

\subsection{Curvelets and Hyperbolic Systems}

The previous section gave a qualitative description of the action of the wave group on a curvelet and we we shall now quantify this fact. The evolution operator $E(t)$ acting on a curvelet $\varphi_{\mu_{0} \nu_{0}}^{(0)}$ is of course not exactly another curvelet $\varphi_{\mu_{0}(t) \nu_{0}}^{(0)}$ which occurs at a displaced location and orientation. Instead, it is a superposition of curvelets $\sum_{\mu, \mu} \alpha_{\mu \nu} \varphi_{\mu \nu}^{(0)}$ such that

1. the coefficients $\left(\alpha_{\mu \nu}\right)$ decay nearly exponentially,

2. and the significant coefficients of this expansion are all located at indices $(\mu, \nu)$ 'near' $\left(\mu_{0}(t), \nu_{0}\right)$. By near, we mean nearby scales, orientations and locations.

To state the key result of this paper, we need a notion of distance $\omega$ between curvelet indices which will be formally introduced in section 2 . Crudely, $\omega\left(\mu, \mu^{\prime}\right)$ is small if and only if both curvelets are at roughly the same scale, have similar orientation and are at nearby spatial locations. In the same spirit, the distance $\omega\left(\mu, \mu^{\prime}\right)$ increases as the distance between the scale, angular, and location parameters increases.

For each $\mu=(j, k, \ell)$ and $\nu=1, \ldots, m$, define the vector-valued curvelets

$$
\boldsymbol{\varphi}_{\mu \nu}=\mathbf{e}_{\nu} \varphi_{\mu}
$$

where $\mathbf{e}_{\nu}$ is the $\nu$ th canonical basis vector in $\mathbb{R}^{m}$. The $\boldsymbol{\varphi}_{\mu \nu}$ 's inherit the tight-frame property (1.12)-(1.13). We would like to again remind the reader that these vector-valued curvelets are simpler and different from the hyper-curvelets $\varphi_{\mu \nu}^{(0)}$ defined in the previous section. Consider now the representing the operator $E(t)$ in a tight-frame of vector-valued curvelets, namely,

$$
E\left(t ; \mu, \nu ; \mu^{\prime}, \nu^{\prime}\right)=\left\langle\boldsymbol{\varphi}_{\mu \nu}, E(t) \boldsymbol{\varphi}_{\mu^{\prime} \nu^{\prime}}\right\rangle .
$$

We will refer to $E\left(t ; \mu, \nu ; \mu^{\prime}, \nu^{\prime}\right)$ or simply $E$ as the curvelet matrix of $E(t)$, with row index $\mu, \nu$ and column index $\mu^{\prime}, \nu^{\prime}$. Decompose the initial wavefield $u_{0}=\sum_{\mu, \nu} c_{\mu \nu} \boldsymbol{\varphi}_{\mu \nu}$. Then one can express the action of $E(t)$ on $u_{0}$ in the curvelet domain as

$$
E(t) u_{0}=\sum_{\mu \nu} c_{\mu \nu}(t) \varphi_{\mu \nu}, \quad c_{\mu \nu}(t)=\sum_{\mu^{\prime}, \nu^{\prime}} E\left(t ; \mu^{\prime}, \nu^{\prime} ; \mu, \nu\right) c_{\mu^{\prime} \nu^{\prime}}
$$


with convergence in $L^{2}\left(\mathbb{R}^{2}, \mathbb{C}^{m}\right)$. In short, the curvelet matrix maps the curvelet coefficients of the initial wavefield $u_{0}(\cdot)$ into those of the solution $u(t, \cdot)$ at time $t$.

Theorem 1.1. Suppose that the coefficients $A_{k}(x)$ and $B(x)$ of the hyperbolic system are $C^{\infty}$ and that the multiplicity of the eigenvalues of the dispersion matrix $\sum_{k} A_{k}(x) \xi_{k}$ is constant in $x$ and $\xi$. Then

- The matrix $E$ is sparse. Suppose a is either a row or a column of $E$, and let $|a|_{(n)}$ be the n-largest entry of the sequence $|a|$, then for each $M>0,|a|_{(n)}$ obeys

$$
|a|_{(n)} \leq C_{t M} \cdot n^{-M}
$$

- The matrix $E$ is well-organized. For each $N>0$, the coefficients obey

$$
\left|E\left(t ; \mu, \nu ; \mu^{\prime}, \nu^{\prime}\right)\right| \leq C_{t N} \cdot \sum_{\nu^{\prime \prime}=1}^{m} \omega\left(\mu, \mu_{\nu^{\prime \prime}}^{\prime}(t)\right)^{-N} .
$$

Here $\mu_{\nu}(t)$ is the curvelet index $\mu$ flown along the $\nu$ th Hamiltonian system.

Both constants $C_{t M}$ and $C_{t N}$ grow in time at most like $C_{1} e^{C_{2} t}$ for some $C_{1}, C_{2}>0$ depending on $M$, resp. $N$.

In effect, the curvelet matrix of the solution operator resembles a sum of $m$ permutation matrices where $m$ is the order of the hyperbolic system; first, there are significant coefficients along $m$ shifted diagonal and second, coefficients away from these diagonals decay nearly exponentially; i.e. faster than any negative polynomial. Now just as wavelets provide sparse representations to the solution operators to certain elliptic differential equations, our theorem shows that curvelets provide an optimally sparse representation of solution operators to systems of symmetric hyperbolic equations.

We can also resort to hyper-curvelets as defined in the previous section and formulate a related result where the curvelet matrix is sparse around a single shifted diagonal. This refinement approximately decouples the evolution into polarized components and will be made precise later.

To grasp the implications of Theorem 1.1, consider the following corollary:

Corollary 1.2. Consider the truncated operator $A_{B}$ obtained by keeping $m \cdot B$ elements per row-the $B$ closest to each shifted diagonal in the sense of the pseudo-distance $\omega$. Then the truncated matrix obeys

$$
\left\|A-A_{B}\right\|_{L^{2} \rightarrow L^{2}} \leq C_{M} \cdot B^{-M}
$$

for each $M>0$.

The proof follows from that of Theorem 1.1 by an application of Schur's lemma and is omitted. Hence, whereas the Fourier or wavelet representations are dense, curvelets faithfully model the geometry of wave propagation as only a few terms are needed to represent the action of the wave group accurately. 


\subsection{Strategy}

In his seminal paper [18], Lax constructed approximate solution operators to linear and symmetric hyperbolic systems, also known as parametrices. He showed that these parametrices are oscillatory integrals in the frequency domain which are commonly referred to as Fourier integral operators (FIO) (the development and study of FIOs is motivated by the connection). An operator $T$ is said to be an FIO if it is of the form

$$
T f(x)=\int e^{i \Phi(x, \xi)} \sigma(x, \xi) \hat{f}(\xi) d \xi .
$$

We suppose the phase function $\Phi$ and the amplitude $\sigma$ obey the following standard assumptions [29]:

- the phase $\Phi(x, \xi)$ is $C^{\infty}$, homogeneous of degree 1 in $\xi$, i.e. $\Phi(x, \lambda \xi)=\lambda \Phi(x, \xi)$ for $\lambda>0$, and with $\Phi_{x \xi}=\nabla_{x} \nabla_{\xi} \Phi$, obeys the nondegeneracy condition

$$
\left|\operatorname{det} \Phi_{x \xi}(x, \xi)\right|>c>0
$$

uniformly in $x$ and $\xi$;

- the amplitude $\sigma$ is a symbol of order $m$, which means that $\sigma$ is $C^{\infty}$, and obeys

$$
\left|\partial_{\xi}^{\alpha} \partial_{x}^{\beta} \sigma(x, \xi)\right| \leq C_{\alpha \beta}(1+|\xi|)^{m-|\alpha|} .
$$

Lax's insight is that the solution of the initial value problem for a variable coefficient hyperbolic system can be well approximated by a superposition of integrals of the form (1.19) with matrix-valued amplitudes of order 0. The phases of these FIO's are, of course, those solving the Eikonal equations (1.7). Hence, a substantial part of our argument will be about proving that curvelets sparsify FIO's. Now an important aspect of this construction is that this approximation is only valid for small times whereas our theorem is valid for all times. The reason is that the solutions to the Eikonal equations (1.7) are not expected to be global in time, because $\Phi_{\nu}$ would become multi-valued when rays originating from the same point $x_{0}$ cross at a later time. This typically happens at cusp points, when caustics start developing. We refer the reader to $[14,33]$. Because, we are interested in a statement valid for all times, we need to bootstrap the construction of the FIO parametrix by composing the small time FIO parametrix with itself. Now this creates an additional difficulty. Each parametrix convects a curvelet along $m$ flows, and we see that after each composition, the number of curvelets would be multiplied by $m$, see section 4.1 for a proper discussion. This would lead to matrices with poor concentration properties. Therefore, the other part of the argument consists in decoupling the equations so that this phenomenon does not occur. In summary, the general architecture of the proof of Theorem 1.1 is as follows:

- We first decompose the wave-field into $m$-one way components, i.e. components which essentially travel along only one flow. We show that this decomposition is sparse in tight-frames of curvelets.

- Second, we show that curvelet representations of FIO's are optimally sparse in tightframe of curvelets, a result of independent interest.

As a side remark, we would like to point out that the result about optimally sparse representations of FIO's was announced without a proof in the companion paper [3]. This paper, however, gives the first proof of this optimality result. 


\subsection{Inspiration and Relation to Other Work}

Underlying our results is a mathematical insight concerning the central role for the analysis of hyperbolic differential equations, played by the parabolic scaling, in which analysis elements are supported in elongated regions obeying the relation width $\approx$ lengt $h^{2}$. In fact, curvelets imply the same tiling of the frequency plane as the Second Dyadic Decomposition (SDD), a construction introduced in the seventies by Stein and Fefferman [15, 29], originally for the purpose of understanding boundedness of Riesz spherical means, and later widely adapted to the study of various Fourier Integral Operators. More specifically, we would like to single out the work of Hart Smith with which we became familiar while working on this project. Smith [26] used parabolic scaling to define function spaces preserved by Fourier Integral Operators [26], and to analyze the behavior of wave equations with low-regularity coefficients [27]. The latter reference actually develops curvelet-like systems which provide a powerful tool to derive so-called sharp Strichartz estimates for solutions to such equations in space dimensions $d=2,3$ (a Strichartz estimate is a bound on the norm of the solution in some appropriate functional space, e.g. $L^{p}$ ). We find the connection with the work of Smith especially stimulating. From a broader viewpoint, the literature on the subject indicates that curvelets are in some sense compatible with a long tradition in harmonic analysis.

The fact that the action of a FIO should be seen as a 'rearrangement of wave packets' was discovered by Córdoba and Fefferman in their visionary paper [10]. They show how simple proofs of $L^{2}$ boundedness and the Garding inequality follow in a straightforward way from the decomposition into wave packets. Without rotations and the parabolic scaling, however, their estimates are not sharp and do not completely capture the geometry of FIO's.

Next, there is of course the inspiration of modern computational harmonic analysis (CHA) whose agenda is the development of orthobases, tight-frames, which are 'optimal' for representing objects (operators, functions) of scientific interest together with rapid algorithms to compute such representations. The point of view here is to develop new mathematical ideas and and turn these ideas into effective algorithms and these effective algorithms into effective and targeted applications. At the beginning of this introduction, we mentioned an instance of this scientific vision: (1) wavelets provide sparse representations of objects with punctuated smoothness and of large classes of singular integrals and other pseudo-differential operators $[22,23] ;(2)$ there are fast discrete wavelet transforms operating in $O(N)$ for a signal of length $N$ [21]; (3) this creates an opportunity for targeted applications in signal processing where wavelets allow for better compression [11], scientific computing where wavelets allow for faster algorithms [2], and for statistical estimation where wavelets allow for sharper reconstructions [12]. This vision was perhaps championed in [2] where (1)-(3) were combined to demonstrate how one can use the wavelet transform to compute certain types of singular integrals in a number of operations of the order of $C(\epsilon) \cdot N \log N$ where $C(\epsilon)$ is a constant depending upon the desired accuracy $\epsilon$.

\subsection{Significance}

We would like to mention how we see our work fit with the vision described above.

- Curvelets and wavefronts. Curvelets are ideal for representing wavefront phenomena [6], or objects which display curve-punctuated smoothness - smoothness except for discontinuity along a general curve with bounded curvature $[4,5]$. This fact originally motivated their construction $[4,5]$. For example, [5] established that curvelets provide 
the sparsest representations of functions which are $C^{2}$ away from piecewise $C^{2}$ edges. Such representations are nearly as sparse as if the object were not singular and turn out to be far more sparse than the wavelet decomposition of the object.

Hence, we see that curvelets provide the unique opportunity for having a representation giving enhanced sparsity of wave groups, and simultaneously of the solution space. We believe that this will eventually be of great practical significance for applications in fields which are great consumers of these mathematical models, e.g. seismics.

- New ideas for new numerical solvers. Clearly, Theorem 1.1 may serve as a basis for faster geometric multiscale PDE solvers. In fact, this paper is the first of a projected series showing how one can exploit the structure of the curvelet transform and the enhanced sparsity of wave groups to derive new numerical low-complexity algorithms for accurately computing the solution to large classes of differential equations, see the concluding section for a discussion.

- Digital curvelet transforms. In order to deploy curvelet-like ideas in practical applications, one would need a digital notion of curvelet transform which (1) would be rapidly computable and (2) would be geometrically faithful in the sense that one would want an accurate digital analog of the corresponding geometric ideas defined at the level of the continuum. There actually is progress on this front. Donoho and one of the authors recently proposed a Digital Curvelet Transform via Unequispaced Fast Fourier Transforms (DCTvUSFFT) [7]. The DCTvUSFFT is a fast algorithm which allows analysis and synthesis of Cartesian arrays as superpositions of discrete curvelets; for practical purposes, the algorithm runs in $O(N \log N)$ operations for input array of size $N$. Digital curvelets obey sharp frequency and spatial localization.

In short, this paper is an essential piece of a much larger body of work.

\subsection{Contents}

Section 2 below reviews the construction of Curvelets. Section 3 below examines secondorder scalar hyperbolic equations and gives a heuristic indicating why the sparsity may be expected to hold. Section 4 links our main result with properties of FIOs. Section 5 proves that FIO's are optimally sparse in scalar curvelet tight-frames. Section 6 discusses implications of this work, namely, in the area of scientific computing. Finally, proofs of key estimates supporting our main result are given in Section 7.

\section{Curvelets}

This section briefly introduces tight frames of curvelets, see [5] for more details.

\subsection{Definition}

We work throughout in $\mathbb{R}^{2}$, with spatial variable $x$, with $\xi$ a frequency-domain variable, and with $r$ and $\theta$ polar coordinates in the frequency-domain. We start with a pair of windows $W(r)$ and $V(t)$, which we will call the 'radial window' and 'angular window', respectively. These are both smooth, nonnegative and real-valued, with $W$ taking positive real arguments 
and supported on $r \in[1 / 2,2]$ and $V$ taking real arguments and supported on $t \in[-1,1]$. These windows will always obey the admissibility conditions:

$$
\begin{aligned}
& \sum_{j=-\infty}^{\infty} W^{2}\left(2^{j} r\right)=1, \quad r>0 \\
& \sum_{\ell=-\infty}^{\infty} V^{2}(t-\ell)=1, \quad t \in \mathbb{R} .
\end{aligned}
$$

Now, for each $j \geq j_{0}$, we introduce $\varphi_{j}\left(x_{1}, x_{2}\right)$ defined in the Fourier domain by

$$
\hat{\varphi}_{j}(\xi)=2^{-3 j / 4} W\left(2^{-j}|\xi|\right) \cdot V\left(2^{\lfloor j / 2\rfloor} \theta\right)
$$

Thus the support of $\hat{\varphi}_{j}$ is a polar 'wedge' defined by the support of $W$ and $V$, the radial and angular windows, applied with scale-dependent window widths in each direction.

We may think of $\varphi_{j}$ as a "mother" curvelet at scale $2^{-j}$ in the sense that all curvelets at that scaled are obtained by rotations and translations of $\varphi_{j}$. Introduce

- the equispaced sequence of rotation angles $\theta_{j, \ell}=2 \pi \cdot 2^{-\lfloor j / 2\rfloor} \cdot \ell, 0 \leq \ell<L_{j}=2^{\lfloor j / 2\rfloor}$,

- and the sequence of translation parameters $k=\left(k_{1}, k_{2}\right) \in \mathbb{Z}^{2}$.

With these notations, we define curvelets (as function of $x=\left(x_{1}, x_{2}\right)$ ) at scale $2^{-j}$, orientation $\theta_{j, \ell}$ and position $b_{k}^{(j, \ell)}=R_{\theta_{j, \ell}}\left(k_{1} \cdot 2^{-j} / \delta_{1}, k_{2} \cdot 2^{-j / 2} / \delta_{2}\right)$ for some adequate constants $\delta_{1}, \delta_{2}$ by

$$
\varphi_{j, k, \ell}(x)=\varphi_{j}\left(R_{-\theta_{j, \ell}}\left(x-b_{k}^{(j, \ell)}\right)\right) .
$$

As in wavelet theory, we also have coarse scale elements. We introduce the low-pass window $W_{0}$ obeying

$$
\left|W_{0}(r)\right|^{2}+\sum_{j \geq 0}\left|W\left(2^{-j} r\right)\right|^{2}=1
$$

and for $k_{1}, k_{2} \in \mathbb{Z}$, define coarse scale curvelets as

$$
\Phi_{j_{0}, k}(x)=\Phi_{j_{0}}\left(x-2^{-j_{0}} k\right), \quad \hat{\Phi}_{j_{0}}(\xi)=2^{-j_{0}} W_{0}\left(2^{-j_{0}}|\xi|\right) .
$$

Hence, coarse scale curvelets are nondirectional. The 'full' curvelet transform consists of the fine-scale directional elements $\left(\varphi_{j, \ell, k}\right)_{j \geq j_{0}, \ell, k}$ and of the coarse-scale isotropic father wavelets $\left(\Phi_{j_{0}, k}\right)_{k}$. For our purposes, it is the behavior of the fine-scale directional elements that matters.

In the remainder of the paper, we will use the generic notation $\left(\varphi_{\mu}\right)_{\mu \in M}$ to index the elements of the curvelet tight-frame. The dyadic-parabolic subscript $\mu$ stands for the triplet $(j, k, \ell)$. We will also make use of the convenient notations

- $x_{\mu}=b_{k}^{(j, \ell)}$ is the center of $\varphi_{\mu}$ in space.

- $\theta_{\mu}=\theta_{j, \ell}$ is the orientation of $\varphi_{\mu}$ with respect to the vertical axis in $x$.

- $\xi_{\mu}=\left(2^{j} \cos \theta_{\mu}, 2^{j} \sin \theta_{\mu}\right)$ is the center of $\hat{\varphi}_{\mu}$ in frequency.

- $e_{\mu}=\xi_{\mu} /\left|\xi_{\mu}\right|$ indicates the codirection of $\varphi_{\mu}$. 


\section{$2.2 \quad$ Properties}

We now list a few properties of the curvelet transform which will play an important role throughout the remainder of this paper.

1. Tight-frame. Much like in an orthonormal basis, we can easily expand an arbitrary function $f\left(x_{1}, x_{2}\right) \in L^{2}\left(\mathbb{R}^{2}\right)$ as a series of curvelets: we have a reconstruction formula

$$
f=\sum_{\mu}\left\langle f, \varphi_{\mu}\right\rangle \varphi_{\mu}
$$

with equality holding in an $L^{2}$ sense; and a Parseval relation

$$
\sum_{\mu}\left|\left\langle f, \varphi_{\mu}\right\rangle\right|^{2}=\|f\|_{L^{2}\left(\mathbb{R}^{2}\right)}^{2}, \quad \forall f \in L^{2}\left(\mathbb{R}^{2}\right) .
$$

2. Parabolic scaling. The frequency localization of $\varphi_{j}$ implies the following spatial structure: $\varphi_{j}(x)$ is of rapid decay away from an $2^{-j}$ by $2^{-j / 2}$ rectangle with minor axis pointing in the horizontal direction. In short, the effective length and width obey the anisotropy scaling relation

$$
\text { length } \approx 2^{-j / 2}, \quad \text { width } \approx 2^{-j} \Rightarrow \text { width } \approx \text { length }^{2} .
$$

3. Oscillatory behavior. As is apparent from its definition, $\hat{\varphi}_{j}$ is actually supported away from the vertical axis $\xi_{1}=0$ but near the horizontal $\xi_{2}=0$ axis. In a nutshell, this says that $\varphi_{j}(x)$ is oscillatory in the $x_{1}$-direction and lowpass in the $x_{2}$-direction. Hence, at scale $2^{-j}$, a curvelet is a little needle whose envelope is a specified 'ridge' of effective length $2^{-j / 2}$ and width $2^{-j}$, and which displays an oscillatory behavior across the main 'ridge'.

4. Phase-Space Tiling/Sampling. We can really think about curvelets as Heisenberg tiles of minimum volume in phase-space. In $x$, the essential support of $\varphi_{\mu}$ has size $O\left(2^{-j} \times 2^{-j / 2}\right)$. In frequency, the support of $\hat{\varphi}_{\mu}$ has size $O\left(2^{j / 2} \times 2^{j}\right)$. The net volume in phase-space is therefore

$$
O\left(2^{-j} \times 2^{-j / 2}\right) \cdot O\left(2^{j / 2} \times 2^{j}\right)=O(1)
$$

which is in accordance with the uncertainty principle. The parameters $(j, k, \ell)$ of the curvelet transform induce a new non-trivial sampling of phase-space, Cartesian in $x$, polar in $\xi$, and based on the parabolic scaling.

5. Complex-valuedness. Since curvelets do not obey the symmetry $\hat{\varphi}_{\mu}(-\xi)=\overline{\hat{\varphi}_{\mu}(\xi)}$, $\varphi_{\mu}$ is complex-valued. There exists a related construction for real-valued curvelets by simply symmetrizing the construction, see [5]. The complex-valued transform is better adapted to the purpose of this paper.

Figure 3 summarizes the key components of the construction. 
Spatial grid: fixed scale and orientation
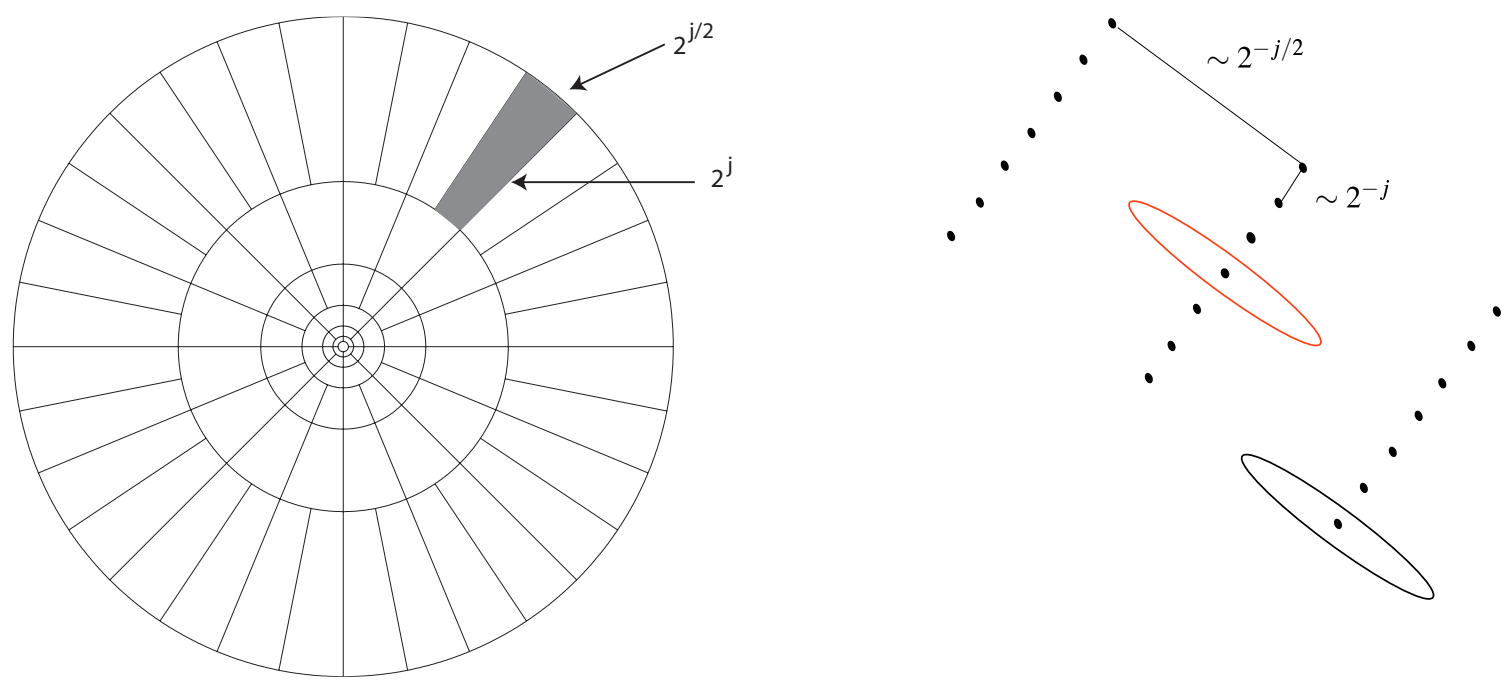

Figure 3: Curvelet tiling of Phase-Space. The figure on the left represents the sampling in the frequency plane. In the frequency domain, curvelets are supported near a 'parabolic' wedge. The shaded area represents such a generic wedge. The figure on the right schematically represents the spatial Cartesian grid associated with a given scale and orientation.

\subsection{Curvelet Molecules}

We introduce the notion of curvelet molecule; our objective, here, is to encompass under this name a wide collection of systems which share the same essential properties as the curvelets we have just introduced. Our formulation is inspired by the notion of 'vaguelettes' in wavelet analysis [23]. Our motivation for introducing this concept is the fact that operators of interest do not map curvelets into curvelets, but rather into these molecules. Note that the terminology 'molecule' is somewhat standard in the literature of harmonic analysis [17].

Definition 2.1. A family of functions $\left(m_{\mu}\right)_{\mu}$ is said to be a family of curvelet molecules with regularity $R$ if (for $j>0$ ) they may be expressed as

$$
m_{\mu}(x)=2^{3 j / 4} a^{(\mu)}\left(D_{2^{-j}} R_{\theta_{\mu}} x-k^{\prime}\right),
$$

where $k^{\prime}=\left(\frac{k_{1}}{\delta_{1}}, \frac{k_{2}}{\delta_{2}}\right)$ and where for all $\mu$, the $a^{(\mu)}$ 's verify the following properties:

- Smoothness and spatial localization: for each $|\beta| \leq R$, and each $M=0,1,2 \ldots$, there is a constant $C_{M}>0$ such that

$$
\left|\partial_{x}^{\beta} a^{(\mu)}(x)\right| \leq C_{M} \cdot(1+|x|)^{-M} .
$$

- Nearly vanishing moments: for each $N=0,1, \ldots, R$, there is a constant $C_{N}>0$ such that

$$
\left|\hat{a}^{(\mu)}(\xi)\right| \leq C_{N} \cdot \min \left(1,2^{-j}+\left|\xi_{1}\right|+2^{-j / 2}\left|\xi_{2}\right|\right)^{N} .
$$

Here, the constants may be chosen independently of $\mu$ so that the above inequalities hold uniformly over $\mu$. There is of course an obvious modification for the coarse scale molecules which are of the form $a^{(\mu)}\left(x-k^{\prime}\right)$ with $a^{(\mu)}$ as in (2.5). 
This definition implies a series of useful estimates. For instance, consider $\theta_{\mu}=0$ so that $R_{\theta_{\mu}}$ is the identity (arbitrary molecules are obtained by rotations). Then, $m_{\mu}$ obeys

$$
\left|m_{\mu}(x)\right| \leq C_{M} \cdot 2^{3 j / 4} \cdot\left(1+\left|2^{j} x_{1}-\frac{k_{1}}{\delta_{1}}\right|+\left|2^{j / 2} x_{2}-\frac{k_{2}}{\delta_{2}}\right|\right)^{-M}
$$

for each $M>0$ and $|\beta| \leq R$, and similarly for its derivatives

$$
\left|\partial_{x}^{\beta} m_{\mu}(x)\right| \leq C_{M} \cdot 2^{3 j / 4} \cdot 2^{\left(\beta_{1}+\beta_{2} / 2\right) j} \cdot\left(1+\left|2^{j} x_{1}-\frac{k_{1}}{\delta_{1}}\right|+\left|2^{j / 2} x_{2}-\frac{k_{2}}{\delta_{2}}\right|\right)^{-M} .
$$

Another useful property is the almost vanishing moments property which says that in the frequency plane, a molecule is localized near the dyadic corona $\left\{2^{j} \leq|\xi| \leq 2^{j+1}\right\} ;\left|\hat{m}_{\mu}(\xi)\right|$ obeys

$$
\left|\hat{m}_{\mu}(\xi)\right| \leq C_{N} \cdot 2^{-3 j / 4} \cdot \min \left(1,2^{-j}(1+|\xi|)\right)^{N},
$$

which is valid for every $N \leq R$, which gives the frequency localization

$$
\left|\hat{m}_{\mu}(\xi)\right| \leq C_{N} \cdot 2^{-3 j / 4} \cdot\left|S_{\mu}(\xi)\right|^{N}
$$

where for $\mu_{0}=(j, 0,0)$,

$$
S_{\mu_{0}}(\xi)=\min \left(1,2^{-j}(1+|\xi|)\right) \cdot\left(1+\left|2^{-j} \xi_{1}\right|+\left|2^{-j / 2} \xi_{2}\right|\right)^{-1} .
$$

For arbitrary $\mu, S_{\mu}$ is obtained from $S_{\mu_{0}}$ by a simple rotation of angle $\theta_{\mu}$, i.e. $S_{\mu_{0}}\left(R_{\theta_{\mu}} \xi\right)$. Similar estimates are available for the derivatives of $\hat{\varphi}_{\mu}$.

In short, a curvelet molecule is a needle whose envelope is supported near a ridge of length about $2^{-j / 2}$ and width $2^{-j}$ and which displays an oscillatory behavior across the ridge. It is easy to show that curvelets as introduced in the previous section are indeed curvelet molecules for arbitrary degrees $R$ of regularity.

\subsection{Near Orthogonality of Curvelet Molecules}

Curvelets are not necessarily orthogonal to each other ${ }^{1}$, but in some sense they are almost orthogonal. As we show below, the inner product between two molecules $m_{\mu}$ and $p_{\mu^{\prime}}$ decays nearly exponentially as a function of the 'distance' between the subscripts $\mu$ and $\mu^{\prime}$.

This notion of distance in phase-space, tailored to curvelet analysis, is to be understood as follows. Given a pair of indices $\mu=(j, k, \ell), \mu^{\prime}=\left(j^{\prime}, k^{\prime}, \ell^{\prime}\right)$, define the dyadic-parabolic pseudo-distance

$$
\omega\left(\mu, \mu^{\prime}\right)=2^{\left|j-j^{\prime}\right|} \cdot\left(1+\min \left(2^{j}, 2^{j^{\prime}}\right) d\left(\mu, \mu^{\prime}\right)\right)
$$

where

$$
d\left(\mu, \mu^{\prime}\right)=\left|\theta_{\mu}-\theta_{\mu^{\prime}}\right|^{2}+\left|x_{\mu}-x_{\mu^{\prime}}\right|^{2}+\left|\left\langle e_{\mu}, x_{\mu}-x_{\mu^{\prime}}\right\rangle\right| .
$$

Angle differences like $\theta_{\mu}-\theta_{\mu^{\prime}}$ are understood modulo $\pi$. As introduced earlier, $e_{\mu}$ is the codirection of the first molecule, i.e., $e_{\mu}=\left(\cos \theta_{\mu}, \sin \theta_{\mu}\right)$.

The pseudo-distance (2.12) is a slight variation on that introduced by Smith [27]. We see that $\omega$ increases by at most a constant factor every time the distance between the scale, angular, and location parameters increases. The extension of the definition of $\omega$ to arbitrary points $(x, \xi)$ and $\left(x^{\prime}, \xi^{\prime}\right)$ is straightforward. Observe that the extra term $\left|\left\langle e_{\mu}, x_{\mu}-x_{\mu^{\prime}}\right\rangle\right|$ induces a non-Euclidean notion of distance between $x_{\mu}$ and $x_{\mu^{\prime}}$. The following properties of $\omega$ are proved in section 7.1. (The notation $A \asymp B$ means that $C_{1} \leq A / B \leq C_{2}$ for some constants $C_{1}, C_{2}>0$.)

\footnotetext{
${ }^{1}$ It is an open problem whether orthobases of curvelets exist or not.
} 
Proposition 2.2. 1. Symmetry: $\omega\left(\mu, \mu^{\prime}\right) \asymp \omega\left(\mu^{\prime}, \mu\right)$.

2. Triangle inequality: $d\left(\mu, \mu^{\prime}\right) \leq C \cdot\left(d\left(\mu, \mu^{\prime \prime}\right)+d\left(\mu^{\prime \prime}, \mu^{\prime}\right)\right)$ for some constant $C>0$.

3. Composition: for every integer $N>0$, and some positive constant $C_{N}$

$$
\sum_{\mu^{\prime \prime}} \omega\left(\mu, \mu^{\prime \prime}\right)^{-N} \cdot \omega\left(\mu^{\prime \prime}, \mu^{\prime}\right)^{-N} \leq C_{N} \cdot \omega\left(\mu, \mu^{\prime}\right)^{-(N-1)} .
$$

4. Invariance under Hamiltonian flows: $\omega\left(\mu, \mu^{\prime}\right) \asymp \omega\left(\mu_{\nu}(t), \mu_{\nu}^{\prime}(t)\right)$.

We can now state the almost orthogonality result

Lemma 2.3. Let $\left(m_{\mu}\right)_{\mu}$ and $\left(p_{\mu^{\prime}}\right)_{\mu^{\prime}}$ be two families of curvelet molecules with regularity $R$. Then for $j, j^{\prime} \geq 0$,

$$
\left|\left\langle m_{\mu}, p_{\mu^{\prime}}\right\rangle\right| \leq C_{N} \cdot \omega\left(\mu, \mu^{\prime}\right)^{-N}
$$

for every $N \leq f(R)$ where $f(R)$ goes to infinity as $R$ goes to infinity.

Proof. Throughout the proof of (2.13), it will be useful to keep in mind that $A \leq C$. $(1+|B|)^{-M}$ for every $M \leq 2 M^{\prime}$ is equivalent to $A \leq C \cdot\left(1+B^{2}\right)^{-M}$ for every $M \leq M^{\prime}$. Similarly, if $A \leq C \cdot\left(1+\left|B_{1}\right|\right)^{-M}$ and $A \leq C \cdot\left(1+\left|B_{2}\right|\right)^{-M}$ for every $M \leq 2 M^{\prime}$, then $A \leq C \cdot\left(1+\left|B_{1}\right|+\left|B_{2}\right|\right)^{-M}$ for every $M \leq M^{\prime}$. Here and throughout, the constants $C$ may vary from expression to expression.

For notational convenience put $\Delta \theta=\theta_{\mu}-\theta_{\mu^{\prime}}$ and $\Delta x=x_{\mu}-x_{\mu^{\prime}}$. We abuse notation by letting $m_{\mu_{0}}$ be the molecule $a^{(\mu)}\left(D_{2^{-j}} R_{\theta_{\mu}} x\right)$, i.e., $m_{\mu_{0}}$ is obtained from $m_{\mu}$ by translation to that it is centered near the origin. Put $I_{\mu \mu^{\prime}}=\left\langle m_{\mu}, p_{\mu^{\prime}}\right\rangle$. In the frequency domain, $I_{\mu \mu^{\prime}}$ is given by

$$
I_{\mu \mu^{\prime}}=\frac{1}{(2 \pi)^{2}} \int \hat{m}_{\mu_{0}}(\xi) \overline{\hat{p}_{\mu_{0}^{\prime}}(\xi)} e^{-i(\Delta x) \cdot \xi} d \xi
$$

Put $j_{0}$ to be the minimum of $j$ and $j^{\prime}$. The Appendix shows that

$$
\int\left|S_{\mu_{0}}(\xi) S_{\mu_{0}^{\prime}}(\xi)\right|^{N} d \xi \leq C \cdot 2^{3 j / 4+3 j^{\prime} / 4} \cdot 2^{-\left|j-j^{\prime}\right| N} \cdot\left(1+2^{j_{0}}|\Delta \theta|^{2}\right)^{-N},
$$

where $S_{\mu_{0}}$ is defined in equation (2.11). Therefore, the frequency localization of the curvelet molecules (2.10) gives

$$
\begin{aligned}
\int\left|\hat{m}_{\mu_{0}}(\xi)\right|\left|\hat{p}_{\mu_{0}^{\prime}}(\xi)\right| d \xi & \leq C \cdot 2^{-3 j / 4-3 j^{\prime} / 4} \cdot \int\left|S_{\mu_{0}}(\xi) S_{\mu_{0}^{\prime}}(\xi)\right|^{N} d \xi \\
& \leq C \cdot 2^{-\left|j-j^{\prime}\right| N} \cdot\left(1+2^{j_{0}}|\Delta \theta|^{2}\right)^{-N} .
\end{aligned}
$$

This inequality explains the angular decay. A series of integrations by parts will introduce the spatial decay, as we now show.

The partial derivatives of $\hat{m}_{\mu}$ obey

$$
\left|\partial_{\xi}^{\alpha} \hat{m}_{\mu}(\xi)\right| \leq C \cdot 2^{-3 j / 4} \cdot 2^{-j\left(\alpha_{1}+\frac{\alpha_{2}}{2}\right)} \cdot\left|S_{\mu}(\xi)\right|^{N} .
$$

Put $\Delta_{\xi}$ to be the Laplacian in $\xi$. Because $\hat{p}_{\mu^{\prime}}$ is misoriented with respect to $e_{\mu}$, simple calculations show that

$$
\begin{aligned}
&\left|\Delta_{\xi} \hat{p}_{\mu^{\prime}}(\xi)\right| \leq C \cdot 2^{-3 j^{\prime} / 4} \cdot 2^{-j^{\prime}} \cdot\left|S_{\mu^{\prime}}(\xi)\right|^{N} \\
&\left|\frac{\partial^{2}}{\partial \xi_{1}^{2}} \hat{p}_{\mu^{\prime}}(\xi)\right| \leq C \cdot 2^{-3 j^{\prime} / 4} \cdot\left(2^{-2 j^{\prime}}+2^{-j^{\prime}}|\sin (\Delta \theta)|^{2}\right) \cdot\left|S_{\mu^{\prime}}(\xi)\right|^{N} .
\end{aligned}
$$


Recall that for $t \in[-\pi / 2, \pi / 2], 2 / \pi \cdot|t| \leq|\sin t| \leq|t|$, so we may just as well replace $|\sin (\Delta \theta)|$ by $|\Delta \theta|$ in the above inequality. Set

$$
L=I-2^{j_{0}} \Delta_{\xi}-\frac{2^{2 j_{0}}}{1+2^{j_{0}}|\Delta \theta|^{2}} \frac{\partial^{2}}{\partial \xi_{1}^{2}}
$$

On the one hand, for each $k, L^{k}\left(\hat{m}_{\mu} \overline{\hat{p}_{\mu^{\prime}}}\right)$ obeys

$$
\left|L^{k}\left(\hat{m}_{\mu} \overline{\hat{p}_{\mu^{\prime}}}\right)(\xi)\right| \leq C \cdot 2^{-3 j / 4-3 j^{\prime} / 4} \cdot\left|S_{\mu}(\xi)\right|^{N} \cdot\left|S_{\mu^{\prime}}(\xi)\right|^{N} .
$$

On the other hand

$$
L^{k} e^{-i(\Delta x) \cdot \xi}=\left[1+2^{j_{0}}|\Delta x|^{2}+\frac{2^{2 j_{0}}}{1+2^{j_{0}}|\Delta \theta|^{2}}\left|\left\langle e_{\mu}, \Delta x\right\rangle\right|^{2}\right]^{k} e^{-i(\Delta x) \cdot \xi} .
$$

Therefore, a few integrations by parts give

$$
\left|I_{\mu \mu^{\prime}}\right| \leq C \cdot 2^{-\left|j-j^{\prime}\right| N} \cdot\left(1+2^{j_{0}}\left|\theta_{\mu}-\theta_{\mu^{\prime}}\right|^{2}\right)^{-N} \cdot\left(1+2^{j_{0}}|\Delta x|^{2}+\frac{2^{2 j_{0}}}{1+2^{j_{0}}|\Delta \theta|^{2}}\left|\left\langle e_{\mu}, \Delta x\right\rangle\right|^{2}\right)^{-N}
$$

and then

$$
\left|I_{\mu \mu^{\prime}}\right| \leq C \cdot 2^{-\left|j-j^{\prime}\right| M} \cdot\left(1+2^{j_{0}}\left(|\Delta \theta|^{2}+|\Delta x|^{2}\right)+\frac{2^{2 j_{0}}}{1+2^{j_{0}}|\Delta \theta|^{2}}\left|\left\langle e_{\mu}, \Delta x\right\rangle\right|^{2}\right)^{-N} .
$$

One can simplify this expression by noticing that

$$
\left(1+2^{j_{0}}|\Delta \theta|^{2}\right)+\frac{2^{2 j_{0}}\left|\left\langle e_{\mu}, \Delta x\right\rangle\right|^{2}}{1+2^{j_{0}}|\Delta \theta|^{2}} \gtrsim \sqrt{1+2^{j_{0}}|\Delta \theta|^{2}} \frac{2^{j_{0}}\left|\left\langle e_{\mu}, \Delta x\right\rangle\right|}{\sqrt{1+2^{j_{0}}|\Delta \theta|^{2}}}=2^{j_{0}}\left|\left\langle e_{\mu}, \Delta x\right\rangle\right| .
$$

This yields equation (2.13) as required.

Remark. Assume that one of the two terms or both terms are coarse scale molecules, e.g. $p_{\mu^{\prime}}$, then the decay estimate is of the form

$$
\left|\left\langle m_{\mu}, p_{\mu^{\prime}}\right\rangle\right| \leq C \cdot 2^{-j N} \cdot\left(1+\left|x_{\mu}-x_{\mu^{\prime}}\right|^{2}+\left|\left\langle e_{\mu}, x_{\mu}-x_{\mu^{\prime}}\right\rangle\right|\right)^{-N} .
$$

For instance, if they are both coarse scale molecules, this would give

$$
\left|\left\langle m_{\mu}, p_{\mu^{\prime}}\right\rangle\right| \leq C \cdot\left(1+\left|x_{\mu}-x_{\mu^{\prime}}\right|\right)^{-N} .
$$

The following result is a different expression for the almost-orthogonality, and will be at the heart of the sparsity estimates for FIO's.

Lemma 2.4. Let $\left(m_{\mu}\right)_{\mu}$ and $\left(p_{\mu}\right)_{\mu}$ be two families of curvelet molecules with regularity $R$. Then for each $p>p^{*}$,

$$
\sup _{\mu} \sum_{\mu^{\prime}}\left|\left\langle m_{\mu}, p_{\mu^{\prime}}\right\rangle\right|^{p} \leq C_{p}
$$

Here $p^{*} \rightarrow 0$ as $R \rightarrow \infty$. In other words, for $p>p^{*}$, the matrix $I_{\mu \mu^{\prime}}=\left(\left\langle m_{\mu}, p_{\mu^{\prime}}\right\rangle\right)_{\mu, \mu^{\prime}}$ acting on sequences $\left(\alpha_{\mu}\right)$ obeys

$$
\|I \alpha\|_{\ell_{p}} \leq C_{p} \cdot\|\alpha\|_{\ell_{p}}
$$


Proof. Put as before $j_{0}=\min \left(j, j^{\prime}\right)$. The appendix shows that

$$
\sum_{\mu \in M_{j^{\prime}}}\left(1+2^{j_{0}}\left(d\left(\mu, \mu^{\prime}\right)\right)^{-N p} \leq C \cdot 2^{2\left|j-j^{\prime}\right|}\right.
$$

provided that $N p>2$. We then have

$$
\sum_{\mu^{\prime}}\left|I_{\mu \mu^{\prime}}\right|^{p} \leq C \cdot \sum_{j^{\prime} \in \mathbb{Z}} 2^{-2\left|j-j^{\prime}\right| N p} \cdot 2^{2\left|j-j^{\prime}\right|} \leq C_{p},
$$

provided again that $N p>2$.

Hence we proved that for $p \leq 1, I$ is a bounded operator from $\ell_{p}$ to $\ell_{p}$. We can of course interchange the role of the two molecules and obtain

$$
\sup _{\mu^{\prime}} \sum_{\mu}\left|\left\langle m_{\mu}, p_{\mu^{\prime}}\right\rangle\right|^{p} \leq C_{p}
$$

For $p=1$, the above expression says that $I$ is a bounded operator from $\ell_{\infty}$ to $\ell_{\infty}$. By interpolation, we then conclude that $I$ is a bounded operator from $\ell_{p}$ to $\ell_{p}$ for every $p$.

\section{Heuristics}

We would like to explain why curvelets are special and why we may expect sparsity by considering the situation of the classical acoustic wave propagation equation (1.4)

$$
\frac{\partial^{2} u}{\partial t^{2}}=c^{2}(x) \Delta u
$$

with initial conditions $u(x, 0)=u_{0}(x), \frac{\partial u}{\partial t}(x, 0)=u_{1}(x)$. To picture the action of the solution operator on a curvelet, consider the case where the velocity is constant, e.g. $c=1$. Put $\hat{u}(\xi, t)$ to be the spatial Fourier transform of the solution at time $t$ (and similarly for $\left.\hat{u}_{0}, \hat{u}_{1}\right)$. In the Fourier domain, the PDE (1.4) is then transformed into an ODE whose solution is given by the relation

$$
\hat{u}(\xi, t)=\cos (|\xi| t) \hat{u}_{0}(\xi)+\frac{\sin (|\xi| t)}{|\xi|} \hat{u}_{1}(\xi) .
$$

To understand the sparsity we need only to consider the sparsity of the multiplication by $\cos (|\xi| t)$, as the exact same arguments apply to the other term. In addition, since

$$
\cos (|\xi| t)=\left(e^{i|\xi| t}+e^{-i|\xi| t}\right) / 2,
$$

we may just as well study the sparsity of the the multiplication by $e^{i|\xi| t}$. We will now explain how the parabolic scaling plays an essential role.

\subsection{Frequency localization}

Because the frequency support of a curvelet $\varphi_{\mu}$ is effectively a thin dyadic rectangle of sidelengths about $2^{j}$ in the direction $e_{\mu}$ and about $2^{j / 2}$ in the orthogonal direction, the modulus of $|\xi|$ is close to the component of $\xi$ along $e_{\mu}$, and we may want to linearize the phase as follows

$$
|\xi|=\xi \cdot e_{\mu}+\left(|\xi|-\xi \cdot e_{\mu}\right)
$$


The point is that the "phase perturbation" $\delta(\xi)=|\xi|-\xi \cdot e_{\mu}$ is bounded over rectangles which support $\hat{\varphi}_{\mu}(\xi)$. With these notations, the phase multiplication takes the form

$$
e^{i|\xi| t} \hat{\varphi}_{\mu}(\xi)=e^{i \delta(\xi) t}\left(e^{i\left(\xi \cdot e_{\mu}\right) t} \hat{\varphi}_{\mu}(\xi)\right)
$$

now the multiplier $e^{i \delta(\xi) t}$ is not really oscillatory (at least for $t$ not too large) thanks to $\delta(\xi)=O(1)$. Therefore, the phase multiplication amounts to a linear modulation followed by a multiplication with a smooth term. The frequency picture is given in figure 4 . In the spatial domain, this simply corresponds to a shift by $t$ along the co-direction of the curvelet followed by a convolution with a gentle kernel.

As it seems reasonable to assume that curvelets sparsify both translations and convolutions, one might expect sparse representations of the solution operator, at least in the case where the coefficients of the differential equations are constant.

\subsection{Spatial localization}

On the one hand, curvelets have enough frequency localization so that they approximately behave like waves. But on the other, they have enough spatial localization so that the flow will essentially preserve their shape, even when the velocity is varying.

At high frequencies or equivalently at small scales, a curvelet has an envelope strongly aligned along a specified 'ridge' of length $2^{-j / 2}$ and width $2^{-j}$ and is oscillatory across the main 'ridge' with a frequency roughly inversely proportional to its width. Now the keypoint is that the velocity field does not vary very much over the support of a curvelet. In fact, owing to the geometric relation width $\sim$ lengt $h^{2}$, the velocity field cannot significantly bend our curvelet. To see this fact, let us model the action of the velocity field as a warping $g(x)$. Consider now the warped curvelet $\varphi_{\mu}(g(x))$. Then the warping leaves the curvelet almost invariant; i.e. our curvelets approximately remain needles essentially fitting in boxes of side-lengths $2^{-j / 2}$ and $2^{-j}$. See figure 5 . This behavior is of course very different from that of classical waves of the form $e^{i x \cdot \xi}$. Because such waves have elongated support, the wavefronts are geometrically distorted.

\subsection{The parabolic scaling is special}

There is something truly remarkable about the parabolic scaling. Instead of curvelets obeying the law

$$
\text { width } \approx \text { length }{ }^{2}
$$

one may want to consider the family of element obeying the more general power-law

$$
\text { width } \approx \text { length }^{\alpha}, \quad 1 \leq \alpha \leq \infty .
$$

However, we have seen that to exhibit the wave-like behavior, one needs enough frequency localization. In our setup, we need that in the frequency domain, our waveform lie in a box of width $\ell$ and length $L$ obeying $\ell^{2} / L=O(1)$. In the spatial domain, this minimum anisotropy imposes the relationship

$$
\text { width } \leq \text { length }{ }^{2} .
$$

But we cannot afford too much anisotropy as otherwise curvelets would be distorted. In the spatial domain, this says that curvelets have to be supported near boxes obeying $L^{2} \leq \ell$ or in other words

$$
\text { width } \geq \text { length } h^{2} \text {. }
$$




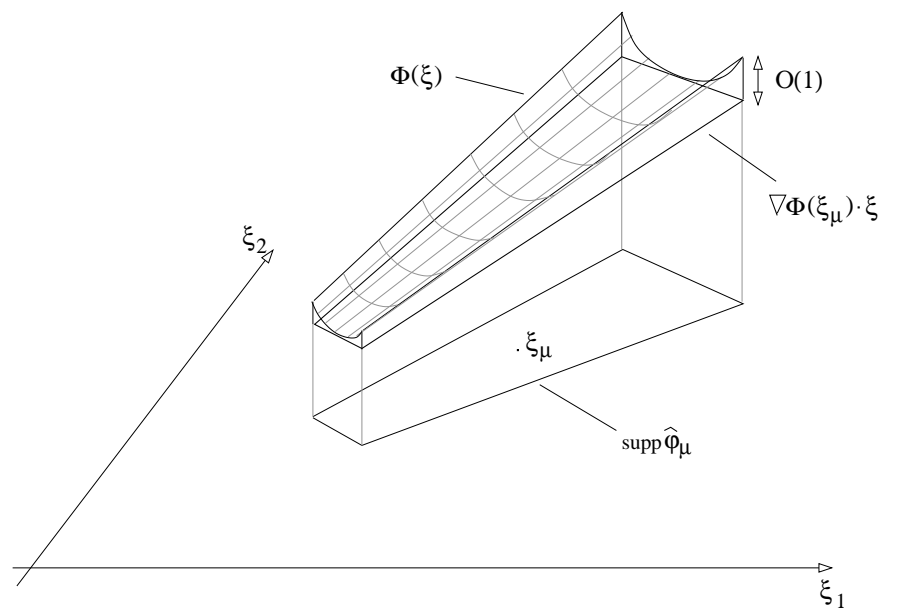

Figure 4: The phase function $\Phi(\xi)$ is wellapproximated by its linearization $\nabla \Phi\left(\xi_{\mu}\right) \cdot \xi$ on the support of $\hat{\varphi}_{\mu}$. In the example discussed in the text, $\Phi(\xi)=t|\xi|$.

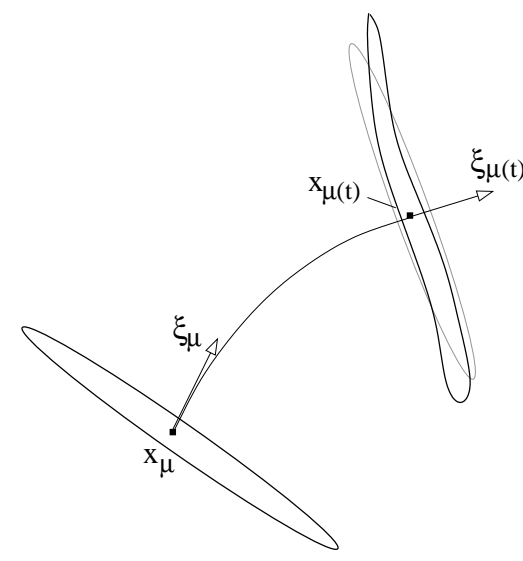

Figure 5: The bottom ellipse indicates the essential support of $\varphi_{\mu}$, initially at $\left(x_{\mu}, \xi_{\mu}\right)$. After propagation, the essential support of $\varphi_{\mu(t)}$ is warped but not by much.

In conclusion, if we want both these properties to hold simultaneously, namely, wave-like behavior and particle-like behavior (no essential distortion by applying a smooth warping), only one scaling may possibly work: the parabolic scaling.

\section{Representation of Linear Hyperbolic Systems}

We now return to the main theme of this paper and consider linear initial-value problems of the form

$$
\frac{\partial u}{\partial t}+\sum_{k=1}^{m} A_{k}(x) \frac{\partial u}{\partial x_{k}}+B(x) u=0, \quad u(0, x)=u_{0}(x),
$$

where in addition to the properties listed in the introduction, $A^{k}$ and $B$ together with all their partial derivatives are uniformly bounded for $x \in \mathbb{R}^{n}$. As explained in section 4.3, we need to make the technical assumption that for every set of real parameters $\xi_{k}$, the (real) eigenvalues of the matrix $\sum_{k} A_{k}(x) \xi_{k}$ have constant multiplicity in $x$ and $\xi$.

Our goal is to construct a concrete 'basis' of $L^{2}\left(\mathbb{R}^{n}, \mathbb{C}^{m}\right)$ in which the evolution is as simple/sparse as possible. We present a solution based on the newly developed curveletswhich were introduced in section 2-and choose to specialize our discussion to $n=2$ spatial dimensions. The reason is twofold: first, this setting is indeed that in which the exposition of curvelets is the most convenient; and second, this is not a restriction as similar results would hold in arbitrary dimensions.

\subsection{Main result}

We need to prove

$$
\left|E\left(t ; \mu, \nu ; \mu^{\prime}, \nu^{\prime}\right)\right| \leq C_{t, N} \cdot \sum_{\nu^{\prime \prime}} \omega\left(\mu, \mu_{\nu^{\prime \prime}}^{\prime}(t)\right)^{-N},
$$


for some constant $C_{t, N}>0$ growing at most like $C_{N} e^{K_{N} t}$ for some $C_{N}, K_{N}>0$. The sum over $\nu^{\prime \prime}$ indexes the different flows and takes on as many values as there are distinct eigenvalues $\lambda_{\nu^{\prime \prime}}^{0}$.

It is instructive to notice that the estimate (4.2) for $t=0$ is already the strongest of its sort on the off-diagonal decay of the Gram matrix elements for a tight frame of curvelets. For $t>0$, equation (4.2) states that the strong phase-space localization of every curvelet is preserved by the hyperbolic system, thus yielding a sparse and well-organized structure for the curvelet matrix. These warped and displaced curvelets are 'curvelet molecules' as introduced in section 2.3 because, as we will show, they obey the estimates (2.5) and (2.6).

The choice of the curvelet family being complex-valued in the above theorem is not essential. $E(t)$ acting on real-valued curvelets would yield two molecules per flow (upstream and downstream). Keeping track of this fact in subsequent discussions would be unnecessarily heavy. In the real case, it is clear that the structure and the sparsity of the curvelet matrix can be recovered by expressing each real curvelet as a superposition of two complex curvelets.

\subsection{Architecture of the proof of the main result}

Much of the remainder of this paper is devoted to the justification of Theorem 1.1 or equivalently (4.2). However, before engaging in the formal proof, we would like to present the overall architecture of the argument.

- Decoupling into polarized components. The first step is to decouple the wavefield $u(t, x)$ into $m$ one-way components $f_{\nu}(t, x)$

$$
u(t, x)=\sum_{\nu=1}^{m} R_{\nu} f_{\nu}(t, x),
$$

where the $R_{\nu}$ are operators mapping scalars to $m$-dimensional vectors, and independent of time. The $f_{\nu}$ will also be called 'polarized' components. This allows a separate study of the $m$ flows corresponding to the $m$ eigenvalues of the matrix $\sum_{k=1}^{m} A_{k}(x) \xi_{k}$. In the event these eigenvalues are simple, the evolution operator $E(t)$ can be decomposed as

$$
E(t)=\sum_{\nu=1}^{m} R_{\nu} e^{-i t \Lambda_{\nu}} L_{\nu}+\text { negligible, }
$$

where the $L_{\nu}$ 's are operators mapping $m$-dimensional vectors to scalars and the $\Lambda_{\nu}$ 's are one-way wave operators acting on scalar functions. In effect, each operator $E_{\nu}(t)=e^{-i t \Lambda_{\nu}}$ convects wave-fronts and other singularities along a separate flow. The 'negligible' contribution is a smoothing operator — not necessarily small. The composition operators $R_{\nu}$ and decomposition operators $L_{\nu}$ are provably pseudo-differential operators, see section 4.3 .

- Fourier Integral Operator parametrix. We then approximate for small times $t>0$ each $e^{-i t \Lambda_{\nu}}, \nu=1, \ldots, m$, by an oscillatory integral or Fourier Integral Operator (FIO) $F_{\nu}(t)$. Such operators take the form

$$
F_{\nu}(t) f(x)=\int e^{i \Phi_{\nu}(t, x, \xi)} \sigma_{\nu}(t, x, \xi) \hat{f}(\xi) d \xi,
$$

under suitable conditions on the phase function $\Phi_{\nu}(t, x, \xi)$ and the amplitude $\sigma_{\nu}(t, x, \xi)$. Again, the identification of the evolution operator $E_{\nu}(t)=e^{-i t \Lambda_{\nu}}$ with $F_{\nu}$ is valid up 
to a smoothing and localized additive remainder. The construction of the so-called parametrix $F_{\nu}(t)$ and its properties are detailed in section 4.4.

Historically [18], the construction of an oscillatory integral parametrix did not involve the decoupling into polarized components as a preliminary step. When applied directly to the system (4.1), the construction of the parametrix gives rise to a matrixvalued amplitude $\sigma(t, x, \xi)$ where all the couplings are present. This somewhat simpler setting, however, is not adequate for our purpose. The reason is that we want to bootstrap the construction of a parametrix to large times by composing the small time FIO parametrix with itself, $F(n t)=[F(t)]^{n}$. Without decoupling of the propagation modes, each $E(t)$ or $F(t)$ involves convection of singularities along $m$ families of characteristics or flows. Applying $F(t)$ again, each flow would artificially split into $m$ flows again, yielding $m^{2}$ fronts to keep track of. At time $T=n t$, that would be at most $m^{n}$ fronts. This flow-splitting situation is not physical and can be avoided by isolating one-way components before constructing the parametrix. The correct largetime argument is to consider $E_{\nu}(n t)$ for small $t>0$ and large integer $n$ as $\left[E_{\nu}(t)\right]^{n}$. This expression involves one single flow, indexed by $\nu$.

- Sparsity of Fourier Integral Operators. The core of the proof is found in section 5 and consists in showing that very general FIO's $F(t)$, including the parametrices $F_{\nu}(t)$, are sparse and well-structured when represented in tight frames of (scalar) curvelets $\varphi_{\mu}$. The scalar analog of Theorem 1.1 for FIO's is Theorem 5.1-a statement of independent interest. Observe that pseudo-differential operators are a special class of FIO's and, therefore, are equally sparse in a curvelet frame.

Section 4.6 assembles key intermediate results and proves Theorem 1.1.

\subsection{Decoupling into polarized components}

How to disentangle the vector wavefield into $m$ independent components is perhaps best understood in the special case of constant coefficients, $A_{k}(x)=A_{k}$, and with $B(x)=0$. In this case, applying the 2-dimensional Fourier transform on both sides of (4.1) gives a system of ordinary differential equations

$$
\frac{d \hat{u}}{d t}(t, \xi)+i a(\xi) \hat{u}(t, \xi)=0, \quad a(\xi)=\sum_{k} A_{k} \xi_{k}
$$

(Note that $a(\xi)$ is a symmetric matrix with real entries.) It follows from our assumptions that one can find $m$ real eigenvalues $\lambda_{\nu}(\xi)$ and orthonormal eigenvectors $r_{\nu}(\xi)$, so that

$$
a(\xi) r_{\nu}(\xi)=\lambda_{\nu}(\xi) r_{\nu}(\xi)
$$

Put $f_{\nu}(t, \xi)=r_{\nu}(\xi) \cdot \hat{u}(t, \xi)$. Then our system of equations is of course equivalent to the system of independent scalar equations

$$
\frac{d f_{\nu}}{d t}(t, \xi)+i \lambda_{\nu}(\xi) f(t, \xi)=0
$$

which can then be solved for explicitly;

$$
f_{\nu}(t, \xi)=e^{-i t \lambda_{\nu}(\xi)} f_{\nu}(0, \xi) .
$$


Hence, the diagonalization of $a(\xi)$ decouples the original equation (4.1) into $m$ polarized components; these can be interpreted as waves going in definite directions, for example 'up and down' or 'outgoing and incoming' depending on the geometry of the problem. This is the reason why $f_{\nu}$ is also referred to as being a 'one-way' wavefield.

The situation is more complicated when $A_{k}(x)$ is non-uniform since Fourier techniques break down. A useful tool in the variable coefficient setting is the calculus of pseudodifferential operators. An operator $T$ is said to be pseudo-differential with symbol $\sigma$ if it can be represented as

$$
T f(x)=\sigma(x, D) f=\frac{1}{(2 \pi)^{2}} \int_{\mathbb{R}^{2}} e^{i x \cdot \xi} \sigma(x, \xi) \hat{f}(\xi) d \xi,
$$

with the convention that $D=-i \nabla$. It is of type $(1,0)$ and order $m$ if $\sigma$ obeys the estimate

$$
\left|\partial_{\xi}^{\alpha} \partial_{x}^{\beta} \sigma(x, \xi)\right| \leq C_{\alpha, \beta} \cdot(1+|\xi|)^{m-|\alpha|}
$$

for every multi-indices $\alpha$ and $\beta$. Unless otherwise stated, all pseudo-differential operators in this paper are of type $(1,0)$. An operator is said to be smoothing of order $-\infty$, or simply smoothing if its symbol satisfies the above inequality for every $m<0$. Observe that this is equivalent to the property that $T$ maps boundedly distributions in the Sobolev space $H^{-s}$ to functions in $H^{s}$ for every $s>0$, in addition to a strong localization property of its kernel $G(x, y)$ which says that for each $N>0$, there is a constant $C_{N}>0$ such that $G$ obeys

$$
|G(x, y)| \leq C_{N} \cdot(1+|x-y|)^{-N}
$$

as in [29][Chapter 6].

Now set

$$
a(x, D)=\sum_{k=1}^{m} A_{k}(x) D_{k}-i B(x)
$$

and its principal part

$$
a^{0}(x, D)=\sum_{k=1}^{m} A_{k}(x) D_{k},
$$

so that equation $(4.1)$ becomes $\partial_{t} u+i a(x, D) u=0$. The matrices $a(x, \xi)$ (resp. $\left.a^{0}(x, \xi)\right)$ are called the symbol of the operator $a(x, D)$ (resp. $\left.a^{0}(x, D)\right)$. Note that $a^{0}(x, \xi)$ is homogeneous of degree one in $\xi ; a^{0}$ also goes by the name of dispersion matrix.

It follows from the symmetry of $A_{k}$ and $B$ that for every set of real parameters $\xi_{1}, \ldots, \xi_{m}$, the matrix $a^{0}(x, \xi)=\sum_{k} A_{k}(x) \xi_{k}$ is also symmetric and thus admits real eigenvalues $\lambda_{\nu}^{0}(x, \xi)$ and an orthonormal basis of eigenvectors $r_{\nu}^{0}(x, \xi)$,

$$
a^{0}(x, \xi) r_{\nu}^{0}(x, \xi)=\lambda_{\nu}^{0}(x, \xi) r_{\nu}^{0}(x, \xi) .
$$

The eigenvalues being real and the set of eigenvectors complete is a hyperbolicity condition and ensures that equation (4.1) will admit wave-like solutions. We assume throughout this paper that the multiplicity of each $\lambda_{\nu}^{0}(x, \xi)$ is constant in $x$ and $\xi$.

By analogy with the special case of constant coefficients, a first impulse may be to introduce the components $r_{\nu}^{0}(x, D) \cdot u$, where $r_{\nu}^{0}(x, D)$ is the operator associated to the eigenvector $r_{\nu}^{0}(x, \xi)$ by the standard rule (4.4). In particular this is how we defined hypercurvelets from curvelets in section 1.4. Unfortunately, this does not perfectly decouple 
the system into $m$ polarized modes - it only approximately decouples. Instead, we would achieve perfect decoupling if we could solve the eigenvalue problem

$$
a(x, D) r_{\nu}(x, D)=r_{\nu}(x, D) \lambda_{\nu}(x, D) .
$$

Here, each $\Lambda_{\nu}=\lambda_{\nu}(x, D)$ is a scalar operator and $R_{\nu}=r_{\nu}(x, D)$ is an $m$-by-1 vector of operators. Equation (4.7) must be understood in the sense of composition of operators. Now let $f_{\nu}$ be the polarized components obeying the scalar equation

$$
\frac{\partial f_{\nu}}{\partial t}+i \Lambda_{\nu} f_{\nu}=0
$$

with initial condition $f_{\nu}(0, x)$ and consider the superposition

$$
u=\sum_{\nu} u_{\nu}, \quad u_{\nu}=R_{\nu} f_{\nu}
$$

Then $u$ is a solution to our initial-value problem (4.1). (We will make this rigorous later, and detail the dependence between the initial values $u_{0}$ and the $f_{\nu}(0, \cdot)$ 's.)

The following result and its proof show how in some cases, (4.7) can be solved up to a smoothing remainder of order $-\infty$. When all the eigenvalues $\lambda_{\nu}^{0}(x, \xi)$ are simple, the exact diagonalization is, in fact, possible. The situation is more complicated when some of the eigenvalues are degenerate. This complication does not compromise, however, any of our results.

Lemma 4.1. Suppose our hyperbolic system satisfies all the assumptions stated below (4.1). Then there exists an $m$-by-m block-diagonal matrix of operators $\Lambda$ and two $m$-by-m matrices of operators $R$ and $S$ such that

$$
a(x, D) R=R \Lambda+S,
$$

where $\Lambda, R$ and $S$ are componentwise pseudo-differential with $\Lambda$ of order one, $R$ of order zero, and $S$ of order $-\infty$. Each block of $\Lambda$ corresponds to a distinct eigenvalue $\lambda_{\nu}^{0}$ whose size equals the multiplicity of that eigenvalue. The principal symbol of $\Lambda$ is diagonal with the eigenvalues $\lambda_{\nu}^{0}(x, \xi)$ as entries.

The lemma says that polarized components corresponding to distinct eigenvalues can be decoupled modulo a smoothing operator. However, further decoupling within the eigenspaces is in general not possible. We would like to point out that a similar result was already given in [31] although in a different context and with a different proof, and was used in [30].

Proof. We already argued (4.7) is not just the eigenvalue problem for the symbol $a(x, \xi)$ for the composition of two operators does not reduce to a multiplication of their respective symbols. Instead, it is common practice [16] to define the twisted product of two symbols $\sigma$ and $\tau$ as

$$
(\sigma \sharp \tau)(x, D)=\sigma(x, D) \tau(x, D),
$$

so that (4.7) becomes the symbol equation $a \sharp r_{\nu}=r_{\nu} \sharp \lambda_{\nu}$. Note that $D=-i \nabla$. The explicit formula for the twisted product is, in multi-index notation ${ }^{2}$,

$$
\sigma \sharp \tau=\sum_{|\alpha| \geq 0} \frac{1}{\alpha !} \partial_{\xi}^{\alpha} \sigma D_{x}^{\alpha} \tau .
$$

\footnotetext{
${ }^{2}$ All the pseudo-differential operators considered in this paper are of type $(1,0)$ therefore all such polyhomogeneous expansions are valid.
} 
We can see that $\sigma \sharp \tau$ is the product $\sigma \tau$ up to terms that are at least one order lower (because of the differentiations in $\xi$ ).

Recall the decomposition of the symbol $a(x, \xi)$ into a principal part $a^{0}(x, \xi)=\sum_{k} A_{k}(x) \xi_{k}$, homogeneous of degree one in $\xi$, and a remainder $B(x)$ homogeneous of order zero. It follows that the eigenvalues $\lambda_{\nu}^{0}(x, \xi)$ of $a^{0}(x, \xi)$ are homogeneous of degree one, and the corresponding eigenvectors $r_{\nu}^{0}(x, \xi)$ may be selected as homogeneous of degree zero (and orthonormal). Up to terms of lower order in $\xi$, the original problem (4.7) therefore reduces to the eigenvalue problem $a^{0}(x, \xi) r_{\nu}^{0}(x, \xi)=r_{\nu}^{0}(x, \xi) \lambda_{\nu}^{0}(x, \xi)$ for the symbol $a^{0}$. It is then natural to look for a solution $r_{\nu}, \lambda_{\nu}$ of (4.7) as a perturbation of $r_{\nu}^{0}, \lambda_{\nu}^{0}$ by lower-order terms.

Consider first the case in which each eigenvalue $\lambda_{\nu}^{0}$ is simple and define the expansions

$$
r_{\nu} \sim r_{\nu}^{0}+r_{\nu}^{1}+r_{\nu}^{2}+\ldots, \quad \lambda_{\nu} \sim \lambda_{\nu}^{0}+\lambda_{\nu}^{1}+\lambda_{\nu}^{2}+\ldots
$$

so that $r_{\nu}^{n}$ is of order $-n$ in $\xi$ and $\lambda_{\nu}^{n}$ of order $-n+1$ i.e.,

$$
\left|\partial_{\xi}^{\alpha} \partial_{x}^{\beta} r_{\nu}^{n}(x, \xi)\right| \leq C_{\alpha, \beta}(1+|\xi|)^{-n-|\alpha|},
$$

and similarly for $\lambda_{\nu}^{n}$. We plug these expansions in the twisted product, or equivalently in (4.7), and isolate terms of identical degree.

The contribution at the leading order is, of course, $a^{0} r_{\nu}^{0}=\lambda_{\nu}^{0} r_{\nu}^{0}$ and the remainder is of the form $a \sharp r_{\nu}^{0}-r_{\nu}^{0} \sharp \lambda_{\nu}^{0}$; put $e_{\nu}^{0}$ as its principal symbol. The zero-order equation reads

$$
\left(a^{0}-\lambda_{\nu}^{0} I\right) r_{\nu}^{1}=-e_{\nu}^{0}+r_{\nu}^{0} \lambda_{\nu}^{1}
$$

which admits a solution if and only if the right hand side has a zero component in the eigenspace spanned by $r_{\nu}^{0}$. This is possible if $\lambda_{\nu}^{1}$ is selected so that

$$
-e_{\nu}^{0}+r_{\nu}^{0} \lambda_{\nu}^{1} \perp r_{\nu}^{0} \Leftrightarrow \lambda_{\nu}^{1}=r_{\nu}^{0} \cdot e_{\nu}^{0}
$$

It follows that equation (4.9) admits the family of solutions

$$
r_{\nu}^{1}=\left(a^{0}-\lambda_{\nu}^{0} I\right)^{-1}\left(-e_{\nu}^{0}+r_{\nu}^{0} \lambda_{\nu}^{1}\right)+f_{1} r_{\nu}^{0}
$$

where $f_{1}$ is actually a scalar function of $x$ and $\xi$, and homogeneous of degree -1 in $\xi$. Our proof does not exploit this degree of freedom.

It is clear that one can successively determine all the $\lambda_{\nu}^{n}$ 's and $r_{\nu}^{n}$ 's in a similar fashion. Let $e_{\nu}^{n}$ be the principal symbol of $a \sharp\left(r_{\nu}^{0}+\ldots+r_{\nu}^{n}\right)-\left(r_{\nu}^{0}+\ldots+r_{\nu}^{n}\right) \sharp\left(\lambda_{\nu}^{0}+\ldots+\lambda_{\nu}^{n}\right)$, then the equation at the order $-n$ is

$$
\left(a^{0}-\lambda_{\nu}^{0} I\right) r_{\nu}^{n+1}=-e_{\nu}^{n}+r_{\nu}^{0} \lambda_{\nu}^{n+1}
$$

and is solved exactly like (4.9).

Suitable cut-offs of the low frequencies guarantee convergence of the series for $r_{\nu}$ and $\lambda_{\nu}$. As is standard in the theory of pseudo-differential operators $[28,32]$, one selects a sequence of $C^{\infty}$ cut-off functions $\chi_{n}(\xi)=\chi\left(\epsilon^{n} \xi\right)$ for some $\chi$ vanishing inside a compact neighborhood of the origin, and identically equal to one outside a larger neighborhood. Then $\epsilon$ is taken small enough so that

$$
r_{\nu}(x, \xi)=\sum_{n=0}^{\infty} r_{\nu}^{n}(x, \xi) \chi_{n}(\xi), \quad \lambda_{\nu}(x, \xi)=\sum_{n=0}^{\infty} \lambda_{\nu}^{n}(x, \xi) \chi_{n}(\xi)
$$


are converging expansions in the topology of $C^{\infty}$. As a result, the remainder $s_{\nu}=a \sharp r_{\nu}-$ $r_{\nu} \sharp \lambda_{\nu}$ also converges to a valid symbol which, by construction, is of order $-\infty$, i.e. obeys

$$
\left|\partial_{\xi}^{\alpha} \partial_{x}^{\beta} s_{\nu}(x, \xi)\right| \leq C_{\alpha, \beta, N} \cdot(1+|\xi|)^{-N-|\alpha|}
$$

for every $N>0$. The lemma is proved in the case when all eigenvalues of the principal symbol are simple.

Consider now the case of a multiple eigenvalue $\lambda^{0}$, say. Suppose the corresponding eigenspace is of dimension $p$ and spanned by $r_{1}^{0}, \ldots, r_{p}^{0}$. The reasoning for simple eigenvalues does not apply because the $p$ solvability conditions are too many for purely diagonal lowerorder corrections. Instead, the block corresponding to $\lambda^{0}$ is now perturbed as

$$
\left(\begin{array}{ccc}
\lambda^{0} & \cdots & 0 \\
\vdots & \ddots & \vdots \\
0 & \cdots & \lambda_{0}
\end{array}\right)+\left(\begin{array}{ccc}
\lambda_{11}^{1} & \cdots & \lambda_{1 p}^{1} \\
\vdots & \ddots & \vdots \\
\lambda_{p 1}^{1} & \cdots & \lambda_{p p}^{1}
\end{array}\right)+\left(\begin{array}{ccc}
\lambda_{11}^{2} & \cdots & \lambda_{1 p}^{2} \\
\vdots & \ddots & \vdots \\
\lambda_{p 1}^{2} & \cdots & \lambda_{p p}^{2}
\end{array}\right)+\ldots
$$

where each $\lambda_{i j}^{n}$ is homogeneous of degree $-n+1$ in $\xi$. At the leading order, The $p$ equations relative to $\lambda_{0}$ are

$$
\left(a^{0}-\lambda^{0} I\right) r_{j}^{1}=-e_{j}^{0}+\sum_{i=1}^{p} r_{i}^{0} \lambda_{i j}^{1},
$$

where $e_{j}^{0}$ is the principal symbol of $a \sharp r_{j}^{0}-r_{j}^{0} \sharp \lambda^{0}$. Solvability requires that the projection of the right-hand side on each of the $r_{i}^{0}, i=1, \ldots, p$ vanishes. This unambiguously determines all the components of the $p$-by- $p$ block $\lambda^{1}$ as

$$
\lambda_{i j}^{1}=r_{i}^{0} \cdot e_{j}^{0} .
$$

All blocks relative to other eigenvalues are solved for in a similar way, yielding a blockdiagonal structure for the zeroth order correction $\lambda^{1}$. Each block should have dimension equal to the multiplicity of the corresponding eigenvalue in order to meet the solvability requirements.

The perturbed eigenvectors $r_{1}^{1}, \ldots, r_{p}^{1}$ are determined as previously once the $\lambda_{i j}^{1}$ are known. The same reasoning applies at all orders and thereby determines $\Lambda$ and $R$. Convergence issues are addressed using cut-off windows just as before.

The above construction indeed provides efficient decoupling of the original problem (4.1) into polarized modes.

Lemma 4.2. In the setting of Lemma 4.1, the solution operator $E(t)$ for (4.1) may be decomposed for all times $t>0$ as

$$
E(t)=R e^{-i t \Lambda} L+\tilde{S}(t),
$$

where the matrices of operators $\Lambda$ and $R$ are defined in Lemma 4.1 and $\tilde{S}(t)$ is (another) matrix of smoothing operators of order $-\infty$. In addition,

1. $L$ is an approximate inverse of $R$, i.e. $R L=I$ and $L R=I$ ( $\bmod$ smoothing).

2. $L$ is a pseudo-differential of order zero (componentwise).

Observe that $e^{-i t \Lambda}$ inherits the block structure from $\Lambda$, and is diagonal in the case where all the eigenvalues $\lambda_{\nu}^{0}$ are simple. 
Proof. Begin by observing that $R=r(x, D)$ - as an operator acting on $L^{2}\left(\mathbb{R}^{2}, \mathbb{C}^{m}\right)$-is invertible modulo a smoothing additive term. This means that one can construct a parametrix $L$ so that $L R=I$ and $R L=I$ with both equations holding modulo a smoothing operator. To see why this is true, note that the matrix $r(x, \xi)$ is a lower-order perturbation from the unitary matrix $r^{0}(x, \xi)$ of eigenvectors of the principal symbol $a^{0}(x, \xi)$. The inverse of $r^{0}(x, \xi)$ is explicitly given by $\ell^{0}(x, \xi)=r^{0}(x, \xi)^{*}$. The symbol of $L$ can now be built as an expansion $\ell^{0}+\ell^{1}+\ldots$, where each $\ell^{n}(x, \xi)$ is homogeneous of degree $-n$ in $\xi$ and chosen to suppress the $O\left(|\xi|^{-j}\right)$ contribution in $R L-I$ as well as $L R-I$. This construction implies that $L$ is pseudo-differential of order zero (componentwise). All of this is routine and detailed in [16][page 117]. An open question is whether $L$ can simply be chosen as the adjoint of $R$.

In the sequel, $S, S_{1}$ and $S_{2}$ will denote a generic smoothing operator whose value may change from line to line. The composition of a pseudo-differential operator and a smoothing operator is obviously still smoothing. Set $f=L u$ and let $A=a(x, D)$, so that $\partial_{t} u=-i A u$. On the one hand, $u=R f-S u$ and

$$
\partial_{t} u=R \partial_{t} f-S \partial_{t} u=R \partial_{t} f-S A u \text {. }
$$

On the other hand, Lemma 4.1 gives

$$
A u=A R f-A S u=R \Lambda f+S_{1} f+S_{2} u=R \Lambda f+S u
$$

Comparing (4.11) and (4.12), and applying $L$ gives

$$
\partial_{t} f=-i \Lambda f+S u \text {. }
$$

This can be solved by Duhamel's formula,

$$
f(t)=e^{-i t \Lambda} f(0)+\int_{0}^{t} e^{-i(t-\tau) \Lambda} S u(\tau) d \tau .
$$

We now argue that the integral term is, indeed, a smoothing operator applied to the initial value $u_{0}$.

- First, the evolution operator $E(t)=e^{-i t A}$ has a kernel $K(t, x, y)$ supported inside a neighborhood of the diagonal $y=x$ and for each $s \geq 0$, is well-known to map $H^{s}\left(\mathbb{R}^{2}, \mathbb{C}^{m}\right)$ boundedly onto itself [18]. Therefore, $S E(\tau)$ maps $H^{-s}$ to $H^{s}$ boundedly for every $s>0$ and has a well-localized kernel in the sense of (4.5). This implies that $S E(\tau)$ is a smoothing operator.

- Second, section 4.4 shows that $e^{-i t \Lambda}$ is, for small $t$, a FIO of type $(1,0)$ and order zero, modulo a smoothing remainder. The composition of a FIO and a smoothing operator is smoothing. For larger $t$, think about $e^{-i t \Lambda}$ as the product $\left(e^{-i \frac{t}{n} \Lambda}\right)^{n}$ for appropriately large $n$.

- And third, the integral extends over a finite interval $[0, t]$ and may be thought as an average of smoothing operator-hence smoothing.

In short, $f(t)=e^{-i t \Lambda} f(0)+S u_{0}$. Applying $R$ on both sides of (4.13) finally gives

$$
u=R e^{-i t \Lambda} L u_{0}+S_{1} u_{0}+S_{2} u=\left(R e^{-i t \Lambda} L+S\right) u_{0}
$$

which is what we set to establish.

It remains to see that the evolution operator $e^{-i t \Lambda}$ for the polarized components has the same block-diagonal structure as $\Lambda$ itself. This is gleaned from equation (4.13): evolution equations for two components $f_{\nu_{1}}, f_{\nu_{2}}$ (corresponding to distinct eigenvalues) are completely decoupled. 


\subsection{The Fourier Integral Operator parametrix}

Lemma 4.2 explained how to turn the evolution operator $E(t)$ into the block-diagonal representation $e^{-i t \Lambda}$. In this section, we describe how each of these blocks can be approximated by a Fourier Integral Operator. The ideas here are standard and our exposition is essentially taken from [14] and [28]. The original construction is due to Lax [18].

Let us first assume that all eigenvalues of the principal symbol $a^{0}(x, \xi)$ are simple. This is the situation where the matrix of operators $\Lambda$ (Lemma 4.1) is diagonal with diagonal elements $\Lambda_{\nu}$. Put $E_{\nu}(t)=e^{-i t \Lambda_{\nu}}$, the (scalar) evolution operator relative to the $\nu$ th polarized mode. We seek a parametrix $F_{\nu}(t)$ such that $S_{\nu}(t)=E_{\nu}(t)-F_{\nu}(t)$ is smoothing of order $-\infty$.

Formally,

$$
f(t, x)=\int E_{\nu}(t)\left(e^{i x \cdot \xi}\right) \widehat{f}_{0}(\xi) d \xi .
$$

Our objective is to build a high-frequency asymptotic expansion for $E_{\nu}(t)\left(e^{i x \cdot \xi}\right)$ of the form

$$
e^{i \Phi_{\nu}(t, x, \xi)} \sigma_{\nu}(t, x, \xi)
$$

where $\sigma_{\nu} \sim \sigma_{\nu}^{0}+\sigma_{\nu}^{1}+\ldots$ and $\sigma_{\nu}^{n}$ is homogeneous of degree $-n$ in $\xi$. As usual, one obtains converging expansions through the use of appropriate low-frequency cutoffs as in the proof of Lemma 4.1.

As is classical in asymptotic analysis, we proceed by applying $M_{\nu}=\partial_{t}+i \Lambda_{\nu}$ to the expansion (4.15) and successively equate all the coefficients of the negative powers of $|\xi|$ to zero, hence mimicking the relation $M_{\nu} E_{\nu}(t)\left(e^{i x \cdot \xi}\right)=0$ which holds by definition. For obvious reasons, we also impose that (4.15) evaluated at $t=0$ be $e^{i x \cdot \xi}$.

We assume that $\Phi_{\nu}$ together with its partial derivatives in $t$ and $x$ be homogeneous of degree one in $\xi$ (note that $\Phi_{\nu}(0, x, \xi)=x \cdot \xi$ ). Next, $\Lambda_{\nu}$ is also expressed as a polyhomogeneous expansion $\Lambda_{\nu} \sim \sum_{j \geq 0} \lambda_{\nu}^{j}(x, D)$, where each symbol $\lambda_{\nu}^{j}(x, \xi)$ is homogeneous of degree $-j+1$ in $\xi$, compare with the proof of Lemma 4.1. In passing, note that $\lambda_{\nu}^{0}(x, \xi)$ is the $\nu$ th eigenvalue of the principal symbol $a^{0}(x, \xi)$, hence our consistent use of notations.

On the one hand,

$$
e^{-i \Phi_{\nu}} \frac{\partial}{\partial t}\left[e^{i \Phi_{\nu}} \sigma_{\nu}\right]=i \frac{\partial \Phi_{\nu}}{\partial t} \sigma_{\nu}+\frac{\partial \sigma_{\nu}}{\partial t} .
$$

On the other hand, the action of $\Lambda_{\nu}$ on the oscillatory product $e^{i \Phi_{\nu}} \sigma_{\nu}$ is more complicated but was studied in $[14,28]$. The following formula is available:

$$
\left.e^{-i \Phi_{\nu}} \Lambda_{\nu}\left[e^{i \Phi_{\nu}} \sigma_{\nu}\right] \sim \sum_{\alpha} \frac{1}{\alpha !} \partial_{\xi}^{\alpha} \lambda_{\nu}\left(x, \nabla_{x} \Phi_{\nu}(t, x, \xi)\right) D_{y}^{\alpha}\left[e^{i q(t, x, y, \xi)} \sigma_{\nu}(t, y, \xi)\right]\right|_{y=x},
$$

where

$$
q(t, x, y, \xi)=\Phi_{\nu}(t, y, \xi)-\Phi_{\nu}(t, x, \xi)-\nabla_{x} \Phi_{\nu}(t, x, \xi) \cdot(y-x) .
$$

Both equations (4.16) and (4.17) are polyhomogeneous expansions. It is rather tedious to show that this property indeed holds for (4.17) and the discussion can be found in [28].

Consider now the leading order relation (coefficient of $|\xi|$ ),

$$
i\left[\frac{\partial \Phi_{\nu}}{\partial t}+\lambda_{\nu}^{0}\left(x, \nabla_{x} \Phi_{\nu}\right)\right] \sigma_{\nu}^{0}=0 .
$$

For nonzero $\sigma_{\nu}^{0}$, the term within brackets must vanish. For each $\nu$, we are thus in the presence of a Hamilton-Jacobi equation

$$
\frac{\partial \Phi_{\nu}}{\partial t}+\lambda_{\nu}^{0}\left(x, \nabla_{x} \Phi_{\nu}\right)=0
$$


which are the well-known equations for the characteristic surfaces of the original problem (4.1). As observed earlier, the initial value is chosen as $\Phi_{\nu}(0, x, \xi)=x \cdot \xi$.

Local existence and uniqueness for (4.18) with the given initial condition is ensured as soon as $a^{0}(x, \xi)$ is $C^{2}$, from the knowledge that $\Phi_{\nu}$ is constant along the bicharacteristics. See also [28]. The $\lambda_{\nu}^{0}$ 's inherit the boundedness and smoothness properties of $a^{0}(x, \xi)$. The solution to (4.18) is not expected to be global in time, because $\Phi_{\nu}$ would become multi-valued when rays originating from the same point $x_{0}$ cross again later. This typically happens at cusp points, when caustics start developing. We refer the reader to [14, 33].

Because the equation (4.18) is homogeneous of degree one in $\Phi_{\nu}$, the degree of homogeneity of $\Phi_{\nu}$ in $\xi$ must match that of the initial condition at all times. This validates our assumption that the phase is of degree one.

Consider the relation at the next order (coefficient of $\left.|\xi|^{0}\right)$,

$$
i\left[\frac{\partial \Phi_{\nu}}{\partial t}+\lambda_{\nu}^{0}\left(x, \nabla_{x} \Phi_{\nu}\right)\right] \sigma_{\nu}^{1}+\frac{\partial \sigma_{\nu}^{0}}{\partial t}+\nabla_{\xi} \lambda_{\nu}^{0}\left(x, \nabla_{x} \Phi_{\nu}\right) \cdot \nabla_{x} \sigma_{\nu}^{0}=-i \lambda_{\nu}^{1}\left(x, \nabla_{x} \Phi_{\nu}\right) \sigma_{\nu}^{0} .
$$

The first term vanishes from (4.18). What remains is a transport equation for $\sigma_{\nu}^{0}$ along the bicharacteristic vector field $\partial_{t}+\nabla_{\xi} \lambda_{\nu}^{0}\left(x, \nabla_{x} \Phi_{\nu}\right) \cdot \nabla_{x}$. This determines $\sigma_{\nu}^{0}$ uniquely from its initial value $\sigma_{\nu}^{0}(0, x, \xi)=1$. It is a smooth function of $x$ and $\xi$, homogeneous of degree zero in $\xi$.

The other $\sigma_{\nu}^{n}$ are determined successively in a very similar fashion. Each relation corresponding to a negative power $-n$ of $|\xi|$ is a transport equation along the same vector field:

$$
\frac{\partial \sigma_{\nu}^{n}}{\partial t}+\nabla_{\xi} \lambda_{\nu}^{0}\left(x, \nabla_{x} \Phi_{\nu}\right) \cdot \nabla_{x} \sigma_{\nu}^{n}=P_{n}\left(\sigma_{\nu}^{0}, \ldots, \sigma_{\nu}^{n}\right),
$$

where $P_{n}$ is a known differential operator applied to $\sigma_{\nu}^{0}, \ldots, \sigma_{\nu}^{n}$. Because of (4.18), the unknown $\sigma_{\nu}^{n+1}$ will always disappear from the relation at the degree $-n$. The initial condition is $\sigma_{\nu}^{n}(0, x, \xi)=0$ for $n>0$. Since by construction, the right-hand side of (4.19) is homogeneous of degree $-n$ in $\xi$, the same holds for $\sigma_{\nu}^{n}$. The smoothness of each $\sigma_{\nu}^{n}$ in $x$ and $\xi$ is inherited from that of the principal symbol $a^{0}(x, \xi)$.

In the case where some eigenvalue $\lambda_{\nu}^{0}$ has multiplicity $p>1$, the construction of a FIO parametrix roughly goes the same way. Let us use the same notation $\Lambda_{\nu}$ to denote the $p$ by- $p$ block corresponding to $\lambda_{\nu}^{0}$ in the matrix $\Lambda$ of Lemma 4.1. The corresponding one-way evolution operator is still denoted by $E_{\nu}(t)=e^{-i t \Lambda_{\nu}}$. We seek a $p$-by- $p$ matrix of operators $F_{\nu}(t)$ approximating $E_{\nu}(t)$. The correct asymptotic expansion for $E_{\nu}(t)\left(e^{i x \cdot \xi}\right)$ is now

$$
e^{i \Phi_{\nu}(t, x, \xi)} \sum_{n=0}^{\infty} \boldsymbol{\sigma}_{\nu}^{n}(t, x, \xi),
$$

where $\Phi_{\nu}$ is a single scalar phase function and each $\sigma_{\nu}^{n}$ is a $p$-by- $p$ matrix, homogeneous of degree $-n$ in $\xi$. Again, we apply $M_{\nu}=I \partial_{t}+i \Lambda_{\nu}$ to this expansion and successively equate identical powers of $|\xi|$ to zero. The leading-order relation is satisfied if and only if $\Phi_{\nu}$ solves (4.18) with initial condition $\Phi_{\nu}(0, x, \xi)=x \cdot \xi$. The next equation, corresponding to $|\xi|^{0}$, is a system of $p$ scalar evolutions equations which determines each column of $\boldsymbol{\sigma}_{\nu}^{0}$ uniquely from the initial condition $\sigma_{\nu}^{0}(0, x, \xi)=I$. This situation repeats itself at all orders and completely determines the expansion.

In all cases the parametrix $F_{\nu}$ thereby defined takes the form of a FIO

$$
F_{\nu}(t) f_{0}(x)=\int e^{i \Phi_{\nu}(t, x, \xi)} \sigma_{\nu}(t, x, \xi) \hat{f}_{0}(\xi) d \xi
$$


with $\Phi_{\nu}$ the phase function, homogeneous of degree one in $\xi$, and $\sigma_{\nu}$ the amplitude, polyhomogeneous of degree 0 in $\xi$. The amplitude is scalar-valued or matrix-valued depending on the multiplicity of $\lambda_{\nu}^{0}$. A suitable choice of cutoff functions in $\xi$ will remove the singular behavior of each $|\xi|^{-n}$ at the origin and guarantees convergence of the series (4.15). This technicality, however, is the reason why $F_{\nu}(t)$ is not exactly equal to $E_{\nu}(t)$ but is only an approximation.

We emphasized that $\Phi_{\nu}$ may be defined only for small times. Put $2 t^{*}$ the infimum time for which a solution to (4.18) ceases to exist, uniformly in $\nu$. In order to establish results for large times, we will simply compose evolution operators; e.g. $E_{\nu}\left(n t^{*}\right)=E_{\nu}\left(t^{*}\right) \ldots E_{\nu}\left(t^{*}\right)$.

Lemma 4.3. Define $t^{*}$ as above. In the setting of lemma 4.1 denote by $\Lambda_{\nu}$ a block of $\Lambda$ and $E_{\nu}(t)=e^{-i t \Lambda_{\nu}}$. Then for every $0<t \leq t^{*}$, there exists a parametrix $F_{\nu}(t)$ for the evolution problem $\partial_{t} f+i \Lambda_{\nu} f=0$, that takes the form of a Fourier Integral Operator. For every such $t$ the phase function $\Phi_{\nu}$ is positive-homogeneous of degree one in $\xi$ and smooth in $x$ and $\xi$; the amplitude $\sigma_{\nu}$ is a symbol of type $(1,0)$ and order zero. The remainder $S_{\nu}(t)=E_{\nu}(t)-F_{\nu}(t)$ is a smoothing operator of order $-\infty$.

Proof. The proof is a slight variation on the argument presented in [28][Pages 120 and below] and is omitted.

\subsection{Sparsity of smoothing terms}

The specialist will immediately recognize that a smoothing operator of order $-\infty$ is very sparse in a curvelet frame. This is the content of the following lemma.

Lemma 4.4. The curvelet entries of a smoothing operator $S$ obey the following estimate: for each $N>0$, there is a constant $C_{N}$ such that

$$
\left|\left\langle\varphi_{\mu}, S \varphi_{\mu^{\prime}}\right\rangle\right| \leq C_{N} \cdot 2^{-\left|j+j^{\prime}\right| N}\left(1+\left|x_{\mu}-x_{\mu^{\prime}}\right|\right)^{-N} .
$$

Note that (4.21) is a stronger estimate than that of Theorem 1.1. Indeed, our lemma implies that

$$
\left|\left\langle\varphi_{\mu}, S \varphi_{\mu^{\prime}}\right\rangle\right| \leq C_{N} \cdot \omega\left(\mu, h_{\nu}\left(t, \mu^{\prime}\right)\right)^{-N}
$$

which is valid for each $N>0$ and regardless of the value of $\nu$.

Proof. We know that $S$ maps $H^{-s}$ to $H^{s}$ for arbitrary large $s$, so does its adjoint $S^{*}$. As a result,

$$
\begin{aligned}
\left|\left\langle\varphi_{\mu}, S \varphi_{\mu^{\prime}}\right\rangle\right| & \leq\left|\left\langle S^{*} \varphi_{\mu}, \varphi_{\mu^{\prime}}\right\rangle\right|^{1 / 2}\left|\left\langle\varphi_{\mu}, S \varphi_{\mu^{\prime}}\right\rangle\right|^{1 / 2} \\
& \leq\left\|S^{*} \varphi_{\mu}\right\|_{H^{s}}^{1 / 2}\left\|\varphi_{\mu^{\prime}}\right\|_{H^{-s}}^{1 / 2}\left\|\varphi_{\mu}\right\|_{H^{-s}}^{1 / 2}\left\|S \varphi_{\mu^{\prime}}\right\|_{H^{s}}^{1 / 2} \\
& \leq C \cdot\left\|\varphi_{\mu^{\prime}}\right\|_{H^{-s}}\left\|\varphi_{\mu}\right\|_{H^{-s}} \leq C \cdot 2^{-\left(j+j^{\prime}\right) s} .
\end{aligned}
$$

On the other hand remember that curvelets have an essential spatial support of size at most $O(1) \times O(1)$, which is the case when $j$ is small (wavelet regime $j=0$ ). The action of $S$ is local on that range of distances, so that

$$
\left|\left\langle\varphi_{\mu}, S \varphi_{\mu^{\prime}}\right\rangle\right| \leq C_{N} \cdot\left(1+\left|x_{\mu}-x_{\mu^{\prime}}\right|\right)^{-N}
$$

for arbitrary large $N>0$. These two bounds can be combined to conclude that the matrix elements of $S$ are negligible in the sense defined above. 


\subsection{Proof of Theorem 1.1}

- Let us first show how the first assertion on the near-exponential decay of the curvelet matrix elements follows immediately from the second one, equation (1.17). Let $a$ be either a row or a column of the curvelet matrix and let $|a|_{(n)}$ be the $n$-largest entry of the sequence $|a|$. We have

$$
n \cdot|a|_{(n)}^{1 / M} \leq\|a\|_{\ell_{1 / M}}
$$

and, therefore, it is sufficient to prove that the matrix $E$ has rows and columns bounded in $\ell_{p}$ for every $p>0$. Consider the columns. We need to establish

$$
\sup _{\mu^{\prime}, \nu^{\prime}} \sum_{\mu, \nu}\left|E\left(t ; \mu, \nu ; \mu^{\prime}, \nu^{\prime}\right)\right|^{p} \leq C_{t, p}
$$

for some constant $C_{t, p}>0$ growing at most like $C_{p} e^{K_{p} t}$ for some $C_{p}, K_{p}>0$.

The sum over $\nu$ and the sup over $\nu^{\prime}$ do not come in the way since these subscripts take on a finite number of values. The fine decoupling between the $m$ one-way components, crucial for equation (1.17), does not play any role here.

Let us now show that there exists $N$, possibly very large, so that

$$
\sum_{\mu} \omega\left(\mu, \mu^{\prime}\right)^{-N p} \leq C_{N, p}
$$

uniformly in $\mu^{\prime}$. For the sum in $k$ and $\ell$ we can use the bound (7.3) with $N p$ in place of $N$, provided $N p$ obeys $N p \geq 2$. What remains is

$$
C_{N, p} \cdot \sum_{j \geq 0} 2^{-\left|j-j^{\prime}\right| N p} \cdot 2^{2\left|j-j^{\prime}\right|},
$$

which is bounded by a constant depending on $N$ and $p$ provided again that $N p \geq 2$.

Hence we proved the property for the columns. The same holds for the rows because the same conclusion is true for the adjoint $E(t)^{*}$; indeed, the adjoint solves the backward initial-value problem for the adjoint equation $u_{t}=A^{*} u$, and $A^{*}$ satisfies the same hyperbolicity conditions as $A$. We can therefore interchange the role of the two curvelets and obtain

$$
\sup _{\mu^{\prime}, \nu^{\prime}} \sum_{\mu, \nu}\left|E\left(t ; \mu^{\prime}, \nu^{\prime} ; \mu, \nu\right)\right|^{p} \leq C_{t, p}
$$

Note that the classical interpolation inequality shows that $E(t)$ is a bounded operator from $\ell_{p}$ to $\ell_{p}$ for every $0<p \leq \infty$.

- We now turn to (1.17). Let us assume first that all eigenvalues $\lambda_{\nu}^{0}$ of the principal symbol $a^{0}$ are simple. According to Lemmas 4.2 and 4.3, each matrix element $E\left(t ; \nu ; \nu^{\prime}\right)=\mathbf{e}_{\nu} \cdot E(t) \mathbf{e}_{\nu^{\prime}}$ of $E(t)$ can for fixed (possibly large) time $t>0$ be written as

$$
E\left(t ; \nu ; \nu^{\prime}\right)=\sum_{\nu^{\prime \prime}=1}^{m} R_{\nu, \nu^{\prime \prime}}\left(e^{-i \frac{t}{n} \Lambda_{\nu^{\prime \prime}}}\right)^{n} L_{\nu^{\prime \prime}, \nu^{\prime}}+S_{\nu, \nu^{\prime}}(t) .
$$

We have taken $n$ large enough-proportional to $t$ - so that $e^{-i \frac{t}{n} \Lambda_{\nu}}$ is a Fourier Integral Operator (mod smoothing) for every $\nu$. Each $R_{\nu, \nu^{\prime}}$ and $L_{\nu, \nu^{\prime}}$ is pseudo-differential of order zero and $S_{\nu, \nu^{\prime}}(t)$ is smoothing. 
Thanks to Lemma 4.4, it is sufficient to establish the theorem about the the first term of (4.23). To do this, we invoke Theorem 5.1 from section 5 about the sparsity of FIO's in a curvelet tight-frame.

It is an interesting exercise to notice that the ray dynamics is equivalently expressed in terms of Hamiltonian flows,

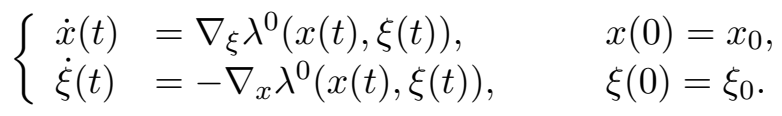

or canonical transformations generated by the phase functions $\Phi_{\nu}$,

$$
\begin{cases}x_{0} & =\nabla_{\xi} \Phi\left(t, x(t), \xi_{0}\right) \\ \xi(t) & =\nabla_{x} \Phi\left(t, x(t), \xi_{0}\right)\end{cases}
$$

provided $\Phi(t, x, \xi)$ satisfies the Hamilton-Jacobi equation $\frac{\partial \Phi}{\partial t}+\lambda^{0}\left(x, \nabla_{x} \Phi\right)=0$ with the initial condition $\Phi\left(0, x, \xi_{0}\right)=x \cdot \xi_{0}$. We obviously need this property to ensure that the geometry of FIO's is the same as that of hyperbolic equations.

Pseudo-differential operators are a special instance of Fourier integral operators so the theorem equally applies to them. For $E\left(t ; \mu, \nu ; \mu^{\prime}, \nu^{\prime}\right)=\left\langle\varphi_{\mu}, E\left(t ; \nu ; \nu^{\prime}\right) \varphi_{\mu}^{\prime}\right\rangle$ we get

$$
\begin{array}{r}
\left|E\left(t ; \mu, \nu ; \mu^{\prime}, \nu^{\prime}\right)\right| \leq C_{N} \sum_{\nu^{\prime \prime}=1}^{m} \sum_{\mu_{0}} \cdots \sum_{\mu_{n}} \omega\left(\mu, \mu_{0}\right)^{-N} \omega\left(\mu_{0}, \mu_{1 \nu^{\prime \prime}}\left(\frac{t}{n}\right)\right)^{-N} \ldots \\
\omega\left(\mu_{n-1}, \mu_{n \nu^{\prime \prime}}\left(\frac{t}{n}\right)\right)^{-N} \omega\left(\mu_{n}, \mu^{\prime}\right)^{-N},
\end{array}
$$

for all $N>0$. Inequality (4.2) then follows from repeated applications of properties 3 and 4 of the distance $\omega$, see proposition 2.2. The power growth in $t$ of the overall multiplicative constant comes from the number of intermediate sums over $\mu_{0}, \ldots, \mu_{n}$. There are $n+1 \sim t$ such sums and they each introduce the same multiplicative constant $C_{N}$.

The reasoning is the same when at least some eigenvalues $\lambda_{\nu}^{0}$ are degenerate. The subscript $\nu^{\prime \prime}$ now denotes the flows i.e., the eigenvalues $\lambda_{\nu^{\prime \prime}}^{0}$ not counting their multiplicity. Each $R_{\nu, \nu^{\prime \prime}}$ is a row vector, $e^{-i \frac{t}{n} \Lambda_{\nu^{\prime \prime}}}$ a matrix and $L_{\nu^{\prime \prime}, \nu^{\prime}}$ a column vector. The FIO parametrix for $e^{-i \frac{t}{n} \Lambda_{\nu^{\prime \prime}}}$ was constructed in such a way that only one flow $h_{\nu^{\prime \prime}}$ appears in the majoration of its curvelet elements (componentwise). There is no intermediate sum over $\nu_{0}, \ldots, \nu_{n}$ and that's the whole point of decoupling the polarized components before constructing the FIO parametrix.

\subsection{Relation to hyper-curvelets}

In section 1.4 we introduced hyper-curvelets as 'polarized' curvelets which would not split into $m$ molecules along the $m$ different flows. In light of section 4.3, it is interesting to reformulate our main result (4.2) in terms of hyper-curvelets. We recall that

$$
\varphi_{\mu \nu}^{(0)}=r_{\nu}^{0}(x, D) \varphi_{\mu}(x)=\frac{1}{(2 \pi)^{2}} \int e^{i x \cdot \xi} r_{\nu}^{0}(x, \xi) \hat{\varphi}_{\mu}(\xi) d \xi
$$

Corollary 4.5. Define $E^{(0)}\left(t ; \mu, \nu ; \mu^{\prime}, \nu^{\prime}\right)=\left\langle\varphi_{\mu \nu}^{(0)}, E(t) \varphi_{\mu^{\prime} \nu^{\prime}}^{(0)}\right\rangle$. Then under the same assumptions as that of theorem 1.1 we have for all $N>0$

$$
\left|E^{(0)}\left(t ; \mu, \nu ; \mu^{\prime}, \nu^{\prime}\right)\right| \leq C_{t N} \cdot\left[\omega\left(\mu, \mu_{\nu^{\prime}}^{\prime}(t)\right)^{-N}+2^{-j^{\prime}} \sum_{\nu^{\prime \prime} \neq \nu^{\prime}} \omega\left(\mu, \mu_{\nu^{\prime \prime}}^{\prime}(t)\right)^{-N}\right] .
$$


The main contribution to the right-hand side of this inequality is the due to the $\nu^{\prime}$ th flow. All other flows are weighted by the small factor $2^{-j^{\prime}}=O\left(|\xi|^{-1}\right)$ on the support of $\hat{\varphi}_{\mu^{\prime}}$. In other words, the leaking of energy from one component $\nu$ to all others when following hyper-curvelets $\varphi_{\mu \nu}^{(0)}$ is smoothing of order -1 , hence small at small scales.

Proof. Equation (4.26) follows from Theorem 1.1 and the fact that the adjoint of the matrix operator $R^{0}$ whose columns are the $R_{\nu}^{0}=r_{\nu}^{0}(x, D)$ is an approximate left inverse for $R^{0}$ up to an error smoothing of order -1 . Indeed, by the standard rules for composition and computation of the adjoint of pseudo-differential operators,

$$
\begin{aligned}
\left(R_{\nu}^{0}\right)^{*} R_{\nu^{\prime}}^{0} & =\left(\left(r_{\nu}^{0}\right)^{*} \sharp r_{\nu^{\prime}}^{0}\right)(x, D) \\
& =\left(\left(r_{\nu}^{0}\right)^{*} r_{\nu^{\prime}}^{0}\right)(x, D)+\text { order }-1 \\
& =\delta_{\nu \nu^{\prime}} I+\text { order }-1 .
\end{aligned}
$$

We have used the fact that the dispersion matrix $a^{0}(x, \xi)$ is assumed to be symmetric, hence admits an orthobasis of eigenvectors $r_{\nu}^{0}(x, \xi)$. We then conclude from Theorem 5.1 applied to pseudo-differential operators of order -1 .

Alternatively, we could have defined hyper-curvelets as

$$
\varphi_{\mu \nu}^{(\infty)}=r_{\nu}(x, D) \varphi_{\mu}(x)=\frac{1}{(2 \pi)^{2}} \int e^{i x \cdot \xi} r_{\nu}(x, \xi) \hat{\varphi}_{\mu}(\xi) d \xi
$$

This would have given the same result. ${ }^{3}$ The reason why we did not use hyper-curvelets in the preceding sections is that they do not necessarily constitute a suitable practical basis to decompose wavefields onto. We do not even know if they always constitute a frame. Digital implementation would also seem less obvious.

\section{Representation of FIOs}

The purpose of this section is to show that Fourier Integral Operators admit a sparse and well-organized structure in a curvelet frame. The main result, Theorem 5.1, is a key step in completing the discussion of the previous section. (Observe that by construction, the FIO's encountered in the previous section satisfy all the assumptions stated in section 1.6 right below (1.19).) As in the previous section, we will restrict the discussion to $x \in \mathbb{R}^{2}$ which is no loss of generality, see section 6 .

\subsection{Main results}

In the introduction section, we detailed a notion of Hamiltonian correspondence for hyperbolic equations. This correspondence also exists for FIO's and is 'encoded' in the phase function $\Phi$ of the FIO. It is called the canonical transformation associated to $\Phi$, and is defined as the mapping $(x, \xi) \rightarrow(y, \eta)$ of phase-space

$$
x=\nabla_{\xi} \Phi(y, \xi), \quad \eta=\nabla_{x} \Phi(y, \xi) .
$$

As suggested in section 4.6, this formulation is equivalent to that involving trajectories along the bicharacteristic flow as in equation (1.6), provided the phase function solves an

\footnotetext{
${ }^{3}$ We can only conjecture that the decoupling should be better if we use the improved $\varphi_{\mu \nu}^{(\infty)}$.
} 
appropriate Hamilton-Jacobi equation. This canonical transformation induces a mapping of curvelet subscripts, denoted by $\mu^{\prime}=h(\mu)$.

The main result for this section reads as follows.

Theorem 5.1. Let $T$ be a Fourier Integral Operator of order $m$ acting on functions of $\mathbb{R}^{2}$, with the assumptions stated above, and $T\left(\mu ; \mu^{\prime}\right)$ denote its matrix elements in the complex curvelet tight frame. Then with $h$ the curvelet index mapping and $\omega$ the distance defined in (2.12), the elements $T\left(\mu ; \mu^{\prime}\right)$ obey for each $N>0$

$$
\left|T\left(\mu ; \mu^{\prime}\right)\right| \leq C_{N} \cdot 2^{m j^{\prime}} \omega\left(\mu, h\left(\mu^{\prime}\right)\right)^{-N},
$$

for some $C_{N}>0$. Moreover, for every $0<p \leq \infty,\left(T\left(\mu, \mu^{\prime}\right)\right)$ is bounded from $\ell^{p}$ to $\ell^{p}$.

The interpretation of Theorem 5.1 is in strong analogy with that of Theorem 1.1. Namely, a FIO has the property of transporting and warping a curvelet into another curvelet-like molecule. (Again, the choice of using complex-valued curvelets is not essential, as a real curvelet would be mapped onto two molecules.)

The proof of Theorem 5.1 relies on the factorization of $T$ on the space-frequency support of $\varphi_{\mu}$ as a nice pseudo-local operator $T_{1, \mu}$ followed by a smooth change of variables, or warping $T_{2, \mu}$. This decomposition goes as follows.

Let $\varphi_{\mu}$ be a fixed curvelet centered around the lattice point $\left(x_{\mu}, \xi_{\mu}\right)$ in phase-space. The phase of our FIO can be decomposed as

$$
\Phi(x, \xi)=\Phi_{\xi}\left(x, \xi_{\mu}\right) \cdot \xi+\delta(x, \xi), \quad \phi_{\mu}(x)=\Phi_{\xi}\left(x, \xi_{\mu}\right) .
$$

In effect, the above decomposition 'linearizes' the frequency variable and is classical, see $[24,29]$. With these notations, we may rewrite the action of $T$ on a curvelet $\varphi_{\mu}$ as

$$
\left(T \varphi_{\mu}\right)(x)=\int e^{i \phi_{\mu}(x) \cdot \xi} e^{i \delta(x, \xi)} \sigma(x, \xi) \hat{\varphi}_{\mu}(\xi) d \xi
$$

Now for a fixed value of the parameter $\mu$, we introduce the decomposition

$$
T=T_{2, \mu} T_{1, \mu},
$$

where

$$
\left(T_{1, \mu} f\right)(x)=\int e^{i x \cdot \xi} b_{\mu}(x, \xi) \hat{f}(\xi) d \xi, \quad\left(T_{2, \mu} f\right)(x)=f\left(\phi_{\mu}(x)\right),
$$

with $\left.b_{\mu}(x, \xi)=e^{i \delta\left(\phi_{\mu}^{-1}(x), \xi\right)} \sigma\left(\phi_{\mu}^{-1}(x), \xi\right)\right)$. This decomposition allows the separate study of the nonlinearities in frequency $\xi$ and space $x$ in the phase function $\Phi$. The point is that both $T_{1, \mu}$ and $T_{2, \mu}$ are sparse in a curvelet tight frame - only for very different reasons.

Theorem 5.2. Let $\left(\varphi_{\mu}\right)_{\mu}$ be a tight frame of curvelets compactly supported in frequency. For each $\mu, T_{1, \mu}$ maps $\varphi_{\mu}$ into a curvelet molecule $m_{\mu}$ with arbitrary regularity $R$, uniformly over $\mu$ in the sense that the constants in estimates (2.5) and (2.6) do not depend on $\mu$.

As we shall see, the proof of Theorem 5.2, presented in section 5.2, relies on the property of compact support in frequency of the $\varphi_{\mu}$. In contrast the corresponding result for the operators $T_{2, \mu}$ which we present next, is extraordinarily simplified if one uses curvelets compactly supported in space. Although well localized in space, the tight frame introduced in section 2 does not meet this requirement. In order to circumvent this technical difficulty, we introduce compactly supported curvelet atoms in section 5.3. They are built on the model of atomic decompositions, standard in approximation theory [17]. 
Theorem 5.3. Let $\left(\rho_{\mu}\right)_{\mu}$ be a family of complex-valued curvelet atoms, compactly supported in space, with regularity $R$. Denote by $h$ the canonical index correspondence associated to $\Phi$, as defined above. For each $\mu, T_{2, \mu}$ maps $\rho_{\mu}$ into a molecule $m_{h(\mu)}$ of the same regularity $R$, uniformly over $\mu$.

The latter theorem says that the 'warped' atom $\rho_{\mu} \circ \phi_{\mu}$ is still an atom, only its scale, orientation, and location may have been changed. That a smooth warping preserves the sparsity of curvelet expansions is a result of independent interest.

The remaining 3 sections are devoted to the proofs of Theorems 5.2, 5.3 and 5.1. The dependence of $\phi_{\mu}$ upon $\mu$ is not essential in proving Theorems 5.2, 5.3 as the only property of interest is that the derivatives of $\phi_{\mu}$ are bounded from above and below uniformly over $\mu$ (which follows from our assumptions about $\Phi$ ). This is the reason why in the next sections we will drop the explicit dependence on $\mu$ and work with a generic warping $\phi$.

\subsection{Proof of Theorem 5.2}

We will assume without loss of generality that our curvelet $\varphi_{\mu}$ is centered near zero $(k=0)$ and is nearly vertical $\left(\theta_{\mu}=0\right)$.

Set $m_{\mu}=T_{1} \varphi_{\mu}$. We first show that $m_{\mu}$ obeys the smoothness and spatial localization estimate of a molecule (2.5). With the same notations as before, recall that $m_{\mu}$ is given by

$$
m_{\mu}(x)=\int e^{i x \cdot \xi} b_{\mu}(x, \xi) \hat{\varphi}_{\mu}(\xi) d \xi, \quad b_{\mu}(x, \xi)=e^{i \delta\left(\phi^{-1}(x), \xi\right)} \sigma\left(\phi^{-1}(x), \xi\right) .
$$

To study the spatial decay of $m_{\mu}(x)$, we introduce the differential operator

$$
L_{\xi}=I-2^{2 j} \frac{\partial^{2}}{\partial \xi_{1}^{2}}-2^{j} \frac{\partial^{2}}{\partial \xi_{2}^{2}},
$$

and evaluate the integral (5.1) using an integration by parts argument. First, observe that

$$
L_{\xi}^{N} e^{i x \cdot \xi}=\left(1+\left|2^{j} x_{1}\right|^{2}+\left|2^{j / 2} x_{2}\right|^{2}\right)^{N} e^{i x \cdot \xi} .
$$

Second, we claim that for every integer $N \geq 0$,

$$
\left|L_{\xi}^{N}\left[b_{\mu}(x, \xi) \hat{\varphi}_{\mu}(\xi)\right]\right| \leq C \cdot 2^{-3 j / 4} .
$$

(The factor $2^{-3 j / 4}$ comes from the $L^{2}$ normalization of $\hat{\varphi}_{\mu}$.) This inequality is proved in appendix 7.2. Hence,

$$
m_{\mu}(x)=\left(1+\left|2^{j} x_{1}\right|^{2}+\left|2^{j / 2} x_{2}\right|^{2}\right)^{-N} \int L_{\xi}^{N}\left[b_{\mu}(x, \xi) \hat{\varphi}_{\mu}(\xi)\right] e^{i x \cdot \xi} .
$$

Since $\left|L_{\xi}^{N}\left[b_{\mu}(x, \xi) \hat{\varphi}_{\mu}(\xi)\right]\right| \leq C \cdot 2^{-3 j / 4}$ and is supported on a dyadic rectangle $R_{\mu}$, of length about $2^{j}$ and width $2^{j / 2}$, we then established that

$$
\left|m_{\mu}(x)\right| \leq C \cdot \frac{2^{3 j / 4}}{\left(1+\left|2^{j} x_{1}\right|^{2}+\left|2^{j / 2} x_{2}\right|^{2}\right)^{N}} .
$$

The derivatives of $m_{\mu}$ are essentially treated in the same way. Begin with

$$
\begin{aligned}
\partial_{x}^{\alpha}\left(e^{i x \cdot \xi} b_{\mu}(x, \xi)\right) & =\sum_{\beta+\varphi \leq \alpha} \partial^{\beta}\left(e^{i x \cdot \xi}\right) \partial^{\varphi}\left(b_{\mu}(x, \xi)\right) \\
& =\sum_{\beta+\varphi \leq \alpha} \partial^{\varphi}\left(b_{\mu}(x, \xi)\right) \xi^{\beta} e^{i x \cdot \xi}
\end{aligned}
$$


Therefore, the partial derivatives of $m_{\mu}$ are given by

$$
\left(\partial_{x}^{\alpha} m_{\mu}\right)(x)=\sum_{\beta+\varphi \leq \alpha} I_{\beta, \varphi}(x)
$$

where

$$
I_{\beta, \varphi}(x)=\int e^{i x \cdot \xi} \partial_{x}^{\varphi}\left(b_{\mu}(x, \xi)\right) \xi^{\beta} \hat{\varphi}_{\mu}(\xi) d \xi .
$$

First, observe that on the support of $\hat{\varphi}_{\mu},|\xi|^{\beta}$ obeys $|\xi|^{\beta} \leq C \cdot 2^{j \beta_{1}} \cdot 2^{j \beta_{2} / 2}$. Second, the term $\partial_{x}^{\varphi} b(x, \xi)$ is of the same nature as $b_{\mu}(x, \xi)$ in the sense that it obeys all the same estimates as before. In particular, we claim that for every integer $N \geq 0$,

$$
\left|L_{\xi}^{N}\left[\partial_{x}^{\varphi} b_{\mu}(x, \xi) \xi^{\beta} \hat{\varphi}_{\mu}(\xi)\right]\right| \leq C \cdot 2^{-3 j / 4} \cdot 2^{j \beta_{1}} \cdot 2^{j \beta_{2} / 2} .
$$

Hence, the same argument as before gives

$$
\left|I_{\beta, \varphi}(x)\right| \leq C \cdot \frac{2^{3 j / 4} \cdot 2^{j \beta_{1}} \cdot 2^{j \beta_{2} / 2}}{\left(1+\left|2^{j} x_{1}\right|^{2}+\left|2^{j / 2} x_{2}\right|^{2}\right)^{N}} .
$$

Now since $\beta \leq \alpha$, we may conclude that

$$
\left|\left(\partial_{x}^{\alpha} m_{\mu}\right)(x)\right| \leq C \cdot \frac{2^{3 j / 4} \cdot 2^{j \alpha_{1}} \cdot 2^{j \alpha_{2} / 2}}{\left(1+\left|2^{j} x_{1}\right|^{2}+\left|2^{j / 2} x_{2}\right|^{2}\right)^{N}} .
$$

This establishes the smoothness and localization property.

The above analysis shows that $m_{\mu}$ is a "ridge" of effective length $2^{-j / 2}$ and width $2^{-j}$; to prove that $m_{\mu}$ is a molecule, we now need to evidence its oscillatory behavior across the ridge. In other words, we are interested in the size of the Fourier transform at low frequencies (2.6)-(2.9).

Formally, the Fourier transform of $m_{\mu}$ is given by

$$
\hat{m}_{\mu}(\xi)=\iint e^{i x \cdot(\eta-\xi)} b_{\mu}(x, \eta) \hat{\varphi}_{\mu}(\eta) d x d \eta
$$

We should point out that because the amplitude $b$ is not of compact support in $x$, the sense in which (5.6) holds is not obvious. This is a well-known phenomenon in Fourier analysis and a classical technique to circumvent such difficulties would be to multiply $m_{\mu}$ (or equivalently $b_{\mu}$ ) by a smooth and compactly supported cut-off function $\chi(\epsilon x)$ and let $\epsilon$ tend to zero. We omit those details as they are standard.

Set $D_{1}=-i \frac{\partial}{\partial x_{1}}$. To develop bounds on $\left|\hat{m}_{\mu}(\xi)\right|$, observe that

$$
D_{1}^{N} e^{i x \cdot \eta}=\left(\eta_{1}\right)^{N}
$$

An integration by parts then gives

$$
\hat{m}_{\mu}(\xi)=\iint e^{i x \cdot \eta} D_{1}^{N}\left(e^{-i x \cdot \xi} b_{\mu}(x, \xi)\right) \eta_{1}^{-N} \hat{\varphi}_{\mu}(\eta) d x d \eta .
$$

Hence,

$$
\hat{m}_{\mu}(\xi)=\sum_{m=0}^{N} c_{m} \xi_{1}^{m} \hat{F}_{m}(\xi)
$$


where

$$
F_{m}(x)=\int e^{i x \cdot \eta}\left(\partial_{x_{1}}^{n-m} b(x, \xi)\right) \eta_{1}^{-n} \hat{\varphi}_{\mu}(\eta) d \eta .
$$

Note that $F_{m}$ is exactly of the same form as (5.4) - but with $\eta_{1}^{-n}$ instead of $\eta^{\beta}$-and therefore, the exact same argument as before gives

$$
\left|F_{m}(x)\right| \leq C \cdot \frac{2^{3 j / 4} \cdot 2^{-j n}}{\left(1+\left|2^{j} x_{1}\right|^{2}+\left|2^{j / 2} x_{2}\right|^{2}\right)^{N}} .
$$

We then established

$$
\left\|\hat{F}_{m}\right\|_{L_{\infty}} \leq\|F\|_{L_{1}} \leq C_{m} \cdot 2^{-3 j / 4} \cdot 2^{-j n}
$$

which gives

$$
\left|m_{\mu}(\xi)\right| \leq C \cdot 2^{-3 j / 4} \cdot 2^{-j n} \cdot\left(1+|\xi|^{n}\right),
$$

as required. This finishes the proof of Theorem 5.2.

The careful reader will object that we did not study the case of coarse scale curvelets; it is obvious that coarse scale elements are mapped into coarse scale molecules and, here, the argument would not require the deployment of the sophisticated tools we exposed above. We omit the proof.

\subsection{Atomic decompositions}

As we will see later, to prove our main result and especially Theorem 5.3, it would be most helpful to work with tight frames of curvelet compactly supported in space. Unfortunately, it is unclear at this point how to construct such tight frames with nice frequency localization properties. However, there exist useful atomic decompositions with compactly supported curvelet-like atoms. We now explore such decompositions.

In this section, the notation $f_{a, \theta}$ refers to the function obtained from $f$ after applying a parabolic scaling and a rotation

$$
f_{a, \theta}(x)=a^{-3 / 4} f\left(D_{a} R_{\theta} x\right), \quad D_{a}=\left(\begin{array}{cc}
1 / a & 0 \\
0 & 1 / \sqrt{a}
\end{array}\right),
$$

and where $R_{\theta}$ is the rotation matrix which maps the vector $(1,0)$ into $(\cos \theta,-\sin \theta)$. Note that this is an isometry as

$$
\left\|f_{a, \theta}\right\|_{L_{2}}=\|f\|_{L_{2}}
$$

In [26], Smith proved the following result: let $\tilde{\psi}$ be a Schwartz function obeying $\hat{\tilde{\psi}}(1,0) \neq$ 0 ; then one can find another Schwartz function $\psi$, and a function $q(\xi)$ such that the following formula holds

$$
q(\xi) \int_{a \leq 1} \hat{\tilde{\psi}}_{a, \theta}(\xi) \hat{\psi}_{a, \theta}(\xi) a d a d \theta=r(\xi)
$$

here $r$ is a smooth cut-off function obeying

$$
r(\xi)=\left\{\begin{array}{ll}
1 & |\xi| \geq 2 \\
0 & |\xi| \leq 1
\end{array},\right.
$$

and $q$ is a standard Fourier multiplier of order zero; that is, for each multiindex $\alpha$, there exists a constant $C_{\alpha}$ such that

$$
\left|\partial_{\xi}^{\alpha} q(\xi)\right| \leq C_{\alpha}(1+|\xi|)^{-|\alpha|}
$$


This formula is useful because it allows us to express any object whose Fourier transform vanishes on $\{|\xi| \leq 2\}$ as a continuous superposition of curvelet-like elements. We now make some specific choices for $\varphi$. In the remainder of this section, we will take $\tilde{\psi}(x)=\psi(-x)$ and the function $\psi$ of the form

$$
\psi\left(x_{1}, x_{2}\right)=\psi^{D}\left(x_{1}\right) \varphi\left(x_{2}\right),
$$

where both $\varphi$ and $\psi^{D}$ are compactly supported and obey

$$
\operatorname{Supp} \varphi \subset[0,1], \quad \operatorname{Supp} \psi^{D} \subset[0,1] .
$$

We will assume that $\varphi$ and $\psi^{D}$ are $C^{\infty}$ and that the function $\psi^{D}$ has vanishing moments up to order $D$, i.e.

$$
\int \psi^{D}\left(x_{1}\right) x_{1}^{k} d x_{1}=0, \quad k=0,1, \ldots, D .
$$

For each $a \leq 1$, each $b \in \mathbb{R}^{2}$ and each $\theta \in[0,2 \pi)$, introduce

$$
\psi_{a, \theta, b}(x):=\psi_{a, \theta}(x-b)=a^{-3 / 4} \psi\left(D_{a} R_{\theta}(x-b)\right) ;
$$

and given an object $f$, define coefficients by

$$
\mathcal{R}(f)(a, b, \theta)=\int \overline{\psi_{a, \theta, b}(x)} f(x) d x .
$$

Now, suppose for instance that $\hat{f}$ vanishes over $|\xi| \leq 2$, then (5.7) gives the exact reconstruction formula

$$
\left.f(x)=\int_{a \geq 1} \mathcal{R}(q(D) f)\right)(a, b, \theta) \psi_{a, \theta, b}(x) \mu(d a d \theta d b),
$$

with $\mu(d a d \theta d b)=a d a d \theta d b$. In the remainder of this section, we will use the shorter notation $d \mu$ for $\mu(d a d \theta d b)$.

As is now well-established, the reproducing formula may be turned into a so-called 'atomic decomposition'. Not surprisingly, our atomic decomposition will just mimic the discretization of the curvelet frame as introduced in section 2 . With the notations of that section, we introduce the cells $Q_{\mu}$ defined as follows: for $j \geq 0, \ell=0,1, \ldots 2^{\lfloor j / 2\rfloor}-1$ and $k=\left(k_{1}, k_{2}\right) \in \mathbb{Z}^{2}$, the cell $Q_{\mu}$ is the collections of triples $(a, \theta, b)$ for which

$$
2^{-(j+1)} \leq a<2^{-j}, \quad\left|\theta-\theta_{\mu}\right| \leq \frac{\pi}{2} 2^{-\lfloor j / 2\rfloor}
$$

and

$$
D_{2^{-j}} R_{\theta_{\mu}} b \in\left[k_{1}, k_{1}+1\right) \times\left[k_{2}, k_{2}+1\right) .
$$

Note that $\int_{Q_{\mu}} d \mu=3 \pi / 2$ for $j$ even, and $3 \pi$ for $j$ odd. We may then break the integral (5.12) into a sum of terms arising from different cells, namely,

$$
f(x)=\sum_{\mu} \alpha_{\mu} \rho_{\mu}(x)
$$

where

$$
\left.\alpha_{\mu}=\|\mathcal{R}(q(D) f)\|_{L_{2}\left(Q_{\mu}\right)}, \quad \rho_{\mu}(x)=\frac{1}{\alpha_{\mu}} \int_{Q_{\mu}} \mathcal{R}(q(D) f)\right)(a, b, \theta) \psi_{a, \theta, b}(x) d \mu .
$$


Of course, the decomposition (5.13) greatly resembles the tight frame expansion, compare (1.5). In particular, the atoms $\rho_{\mu}$ are curvelet-like in the sense that they share all the properties of the tight frame $\left(\varphi_{\mu}\right)_{\mu}$ - only they are compactly supported in space. In the remainder of the paper, we will call these elements atoms. Below are some crucial properties of these atoms.

Lemma 5.1. Rewrite the atoms $\rho_{\mu}$ as $\rho_{\mu}(x)=2^{3 j / 4} a^{(\mu)}\left(D_{2^{-j}} R_{\theta_{\mu}} x-k\right)$. In other words, $\rho_{\mu}$ is obtained from $a^{(\mu)}$ after parabolic scaling, rotation, and translation. For all $\mu$, the $a^{(\mu)}$ 's verify the following properties.

- Compact support;

$$
\text { Supp } a^{(\mu)} \subset c Q \text {. }
$$

- Nearly vanishing moment along the horizontal axis; let $m=D / 2$. Then for each $k=0,1, \ldots, m$, there is a constant $C_{m}$ such that

$$
\int a^{(\mu)}\left(x_{1}, x_{2}\right) x_{1}^{k} d x_{1} \leq C_{m} \cdot 2^{-j(m+1)} .
$$

- Regularity; for every multiindex $\alpha$

$$
\left|\partial_{x}^{\alpha} a^{(\mu)}(x)\right| \leq C_{\alpha}
$$

In (5.16) and (5.17), the constants may be chosen independently of $\mu$ and $f$.

Proof. See appendix 7.2

Needless to say that curvelet atoms are molecules with spatial compact support, compare lemma 5.1 with the definition of a molecule. Finally, observe (and this is important) that it is of course possible to decompose a molecule into a series of atoms

$$
m_{\mu}=\sum_{\mu^{\prime}} \alpha_{\mu \mu^{\prime}} \rho_{\mu^{\prime}}
$$

The coefficients would then obey the same estimate as in lemma 2.3

$$
\left|\alpha_{\mu \mu^{\prime}}\right| \leq C_{N} \cdot \omega\left(\mu, \mu^{\prime}\right)^{-N}
$$

and in particular, for each $p>0$,

$$
\sup _{\mu} \sum_{\mu^{\prime}}\left|\alpha_{\mu \mu^{\prime}}\right|^{p}<C_{p}
$$

This is briefly justified in appendix 7.2.

\subsection{Proof of Theorem 5.3}

As mentioned earlier, curvelet atoms depend in a nonessential way upon the object $f$ we wish to analyze and we shall drop this dependence in our notations. To prove Theorem 5.3, recall that we need to show that for each curvelet atom $\rho_{\mu}$ with regularity $R$, the 'warped' atom $\rho_{\mu} \circ \phi$ is also a curvelet atom, with the same regularity. 
As in Section 5.3, we suppose our curvelet atom is of the form

$$
\rho_{\mu}(x)=2^{3 j / 4} a^{(\mu)}\left(D_{2^{-j}} R_{\theta_{\mu}}\left(x-x_{\mu}\right)\right),
$$

where $a^{(\mu)}$ obeys the conditions of Lemma 5.1. (Here, the location $x_{\mu}$ may be formally defined by $x_{\mu}=\left(D_{2^{-j}} R_{\theta_{\mu}}\right)^{-1} k_{\delta}$. $)$ Define $y_{\mu}$ and $A_{\mu}$ by

$$
y_{\mu}=\phi^{-1}\left(x_{\mu}\right), \quad \text { and } \quad A_{\mu}=(\nabla \phi)\left(y_{\mu}\right)
$$

so that

$$
\phi(y)=x_{\mu}+A_{\mu}\left(y-y_{\mu}\right)+g\left(y-y_{\mu}\right) .
$$

With these notations, it is clear that the warped atom $\rho_{\mu} \circ \phi$ will be centered near the point $y_{\mu}$; that is,

$$
\rho_{\mu}(\phi(y))=2^{3 j / 4} a^{(\mu)}\left(D_{2^{-j}} R_{\theta_{\mu}}\left(A_{\mu}\left(y-y_{\mu}\right)+g\left(y-y_{\mu}\right)\right)\right) .
$$

To simplify matters, we first assume that $A_{\mu}$ is the identity and show that $\rho_{\mu} \circ \phi$ is a curvelet atom with the same scale and orientation as $\rho_{\mu}$. Later, we will see that in general, $\rho_{\mu} \circ \phi$ is an atom whose orientation depends upon $A_{\mu}$, and whose scale may be taken to be the same as that of $\rho_{\mu}$. Assume without loss of generality that $\theta_{\mu}=0$ and $y_{\mu}=0$ (statements for arbitrary orientations and locations are obtained in an obvious fashion) so that

$$
\rho_{\mu}(\phi(y))=2^{3 j / 4} a^{(\mu)}\left(D_{2^{-j}}(y+g(y))\right)=2^{3 j / 4} b^{(\mu)}\left(D_{2^{-j}} y\right),
$$

with

$$
b^{(\mu)}(y)=a^{(\mu)}\left(y+D_{2^{-j}} g\left(D_{2^{j}} y\right)\right) .
$$

The atom $a^{(\mu)}$ is supported over a square of sidelength about 1 ; likewise, $b^{(\mu)}$ is also compactly supported in a box of roughly the same size - uniformly over $\mu$. We then need to derive smoothness estimates and show that $b^{(\mu)}$ obeys

$$
\left|\partial^{\alpha} b^{(\mu)}(y)\right| \leq C_{\alpha}, \quad|\alpha| \leq R
$$

Over the support of $\rho_{\mu} \circ \phi, g=\left(g_{1}, g_{2}\right)$ deviates little from zero and for each $k=1,2, g_{k}$ obeys

$$
\left|g_{k}(y)\right| \leq C \cdot 2^{-j}, \quad\left|\partial^{\alpha} g_{k}(y)\right| \leq C \cdot 2^{-j / 2},|\alpha|=1 .
$$

Similarly, for each $\alpha,|\alpha|>1$,

$$
\left|\partial^{\alpha} g_{k}(y)\right| \leq C_{\alpha}
$$

These estimates hold uniformly over $\mu$. It follows that for $\left|y_{1}\right|,\left|y_{2}\right| \leq C$ and each $\alpha$, the perturbation $g$ obeys

$$
2^{j} \cdot\left|\partial^{\alpha} g_{1}\left(2^{-j} y_{1}, 2^{-j / 2} y_{2}\right)\right| \leq C_{\alpha}, \quad 2^{j / 2} \cdot\left|\partial^{\alpha} g_{2}\left(2^{-j} y_{1}, 2^{-j / 2} y_{2}\right)\right| \leq C_{\alpha} .
$$

The bound (5.21) is then a simple consequence of (5.23) together with the fact that all the derivatives of $a^{(\mu)}$ up to order $R$ are bounded, uniformly over $\mu$.

We now show that $\rho_{\mu} \circ \phi$ exhibits the appropriate behavior at low frequencies.

$$
\begin{aligned}
\widehat{\rho_{\mu} \circ \phi}(\xi) & =\int e^{-i x \cdot \xi} \rho_{\mu}(\phi(x)) d x \\
& =\int e^{-i \phi^{-1}(x) \cdot \xi} \rho_{\mu}(x) \frac{d x}{|\operatorname{det} \nabla \phi|\left(\phi^{-1}(x)\right)} .
\end{aligned}
$$


We will use the nearly vanishing moment property of $\rho_{\mu}$. Set

$$
S_{\xi}(x)=e^{-i \phi^{-1}(x) \cdot \xi} /|\operatorname{det} \nabla \phi|\left(\phi^{-1}(x)\right)
$$

note that over the support of $\rho_{\mu}$ and for each $N \leq R$, we have available the following upper bound on the partial derivative of $S_{\xi}$

$$
\left|\partial_{1}^{N} S_{\xi}(x)\right| \leq C_{N} \cdot(1+|\xi|)^{N}
$$

Classical arguments give

$$
\widehat{\rho_{\mu} \circ \phi}(\xi)=\sum_{k=0}^{n-1} \int \frac{\partial_{1}^{k} S_{\xi}\left(0, x_{2}\right)}{k !} d x_{2} \int \rho_{\mu}\left(x_{1}, x_{2}\right) x_{1}^{k} d x_{1} d x_{2}+E,
$$

where $E$ is a remainder term obeying

$$
|E| \leq C_{n} \cdot 2^{-3 j / 4} \cdot 2^{-j n} \cdot \sup \left|\partial_{1}^{n} S_{\xi}(x)\right| \leq C_{n} \cdot 2^{-3 j / 4} \cdot 2^{-j n}\left(1+|\xi|^{n}\right) .
$$

The near-vanishing moment property gives that each term in the right-hand side of (5.24) obeys the estimate in (5.25). This proves that the Fourier transform of $\rho_{\mu} \circ \phi$ obeys

$$
\left|\widehat{\rho_{\mu} \circ \phi}(\xi)\right| \leq C_{n} \cdot 2^{-j n}\left(1+|\xi|^{n}\right)
$$

as required.

We now discuss the case where the matrix $A_{\mu}$ is not the identity. In this case, (5.20) becomes

$$
\rho_{\mu}(\phi(y))=m_{\mu}\left(A_{\mu} y\right)
$$

with

$$
m_{\mu}(y)=2^{3 j / 4} a^{(\mu)}\left(D_{2^{j}}(y+\tilde{g}(y))\right), \quad \text { and } \quad \tilde{g}(y)=g\left(A_{\mu}^{-1} y\right) .
$$

Our assumptions about FIOs guarantee that $\left|A_{\mu}^{-1}\right|$ is uniformly bounded and, therefore, it follows from the previous analysis that $m_{\mu}$ is a curvelet atom. As a consequence $\rho_{\mu} \circ$ $\phi$ is a curvelet atom with the same regularity $R$ since it is clear that bounded linear transformations of the plane map curvelet atoms into curvelet atoms.

\subsection{Proof of Theorem 5.1}

Let $\varphi_{\mu_{0}}$ be a fixed curvelet and decompose $T$ as $T_{2, \mu_{0}} \circ T_{1, \mu_{0}}$. First, Theorem 5.2 proved that $T_{1, \mu_{0}} \varphi_{\mu_{0}}$ is a curvelet molecule $m_{\mu_{0}}$ which we will express as a superposition of curvelet atoms $\rho_{\mu_{1}}$

$$
T_{1, \mu_{0}} \varphi_{\mu_{0}}=m_{\mu_{0}}=\sum_{\mu_{1}} \beta_{0}\left(\mu_{1}, \mu_{0}\right) \rho_{\mu_{1}} .
$$

Second, for each $\mu_{1}$, Theorem 5.3 shows that $T_{2, \mu_{1}} \rho_{\mu_{1}}$ is a molecule $m_{h\left(\mu_{1}\right)}$ at the location $h\left(\mu_{1}\right)$. We are not exactly in that setting since in $T_{2, \mu_{0}} \rho_{\mu_{1}}$, the subscripts do not in general match. This does not pose any difficulty since Theorem 5.3 can be understood as a statement concerning general warpings $\phi$. We can define the map $h_{\mu_{0}}$ as induced by the transformation $(x, \xi) \rightarrow(y, \eta)$ given by

$$
x=\nabla_{x} \Phi\left(y, \xi_{\mu_{0}}\right), \quad \quad \eta=\nabla_{\xi}\left(y, \xi_{\mu_{0}}\right)
$$


(compare this with equation (5.1)). Then, according to Theorem 5.3, $T_{2, \mu_{0}} \rho_{\mu_{1}}$ is a molecule $m_{h_{\mu_{0}}\left(\mu_{1}\right)}$ at the location $h_{\mu_{0}}\left(\mu_{1}\right)$. So

$$
\left\langle\varphi_{\mu_{2}}, T_{2, \mu_{0}} \rho_{\mu_{1}}\right\rangle=\beta_{1}\left(\mu_{2}, h_{\mu_{0}}\left(\mu_{1}\right)\right)
$$

Hence,

$$
\left\langle\varphi_{\mu_{2}}, T \varphi_{\mu_{0}}\right\rangle=\sum_{\mu_{1}} \beta_{1}\left(\mu_{2}, h_{\mu_{0}}\left(\mu_{1}\right)\right) \beta_{0}\left(\mu_{1}, \mu_{0}\right) .
$$

Of course, both $\beta_{0}$ and $\beta_{1}$ obey very special decay properties.

- By Theorem 5.2 and Lemma $2.3,\left|\beta_{0}\left(\mu_{1}, \mu_{0}\right)\right| \leq C_{n} \cdot \omega\left(\mu_{1}, \mu_{0}\right)^{-N}$ for arbitrarily large $N>0$, provided that the selected atoms are regular enough.

- By Theorem 5.3 and Lemma $2.3,\left|\beta_{1}\left(\mu_{2}, h_{\mu_{0}}\left(\mu_{1}\right)\right)\right| \leq C_{N} \cdot \omega\left(\mu_{2}, h_{\mu_{0}}\left(\mu_{1}\right)\right)^{-N}$ for arbitrarily large $N>0$, provided that the selected atoms are regular enough.

Theorem 5.1 now follows from the observation that

$$
\sum_{\mu_{1}} \omega\left(\mu_{2}, h_{\mu_{0}}\left(\mu_{1}\right)\right)^{-N} \cdot \omega\left(\mu_{1}, \mu_{0}\right)^{-N} \leq C_{n} \cdot \omega\left(\mu_{2}, h_{\mu_{0}}\left(\mu_{0}\right)\right)^{-(N-1)},
$$

This is an immediate consequence of properties 3 and 4 of the pseudo-distance $\omega$, see proposition 2.2 .

Cases involving coarse scale elements are treated similarly and we omit the proof. The boundedness from $\ell^{p}$ to $\ell^{p}$ for every $p>0$ follows from the same argument as in the proof of Theorem 1.1.

\section{Discussion}

All along we specialized our discussion to the special case where the dimension of the spatial variable is $n=2$. It is clear that nothing in our arguments depends upon this specific assumption. Indeed, we could just as well construct tight-frames of curvelets in arbitrary dimensions by smoothly partitioning the frequency plane into dyadic coronae, which would then be angularly localized near regions of sidelength length $2^{j}$ in the radial direction and $2^{j / 2}$ in all the other directions; in order to this, we would use smooth partitions of the unit sphere of $\mathbb{R}^{n}$ into spherical caps of radius about $2^{-j / 2}$. All of our analysis would apply as is, and would prove versions of Theorem 1.1 in arbitrary dimensions.

Our main result assumes that the coefficients of the equation (1.1) be smooth. In many applications of interest, however, the coefficients may be smooth away from singular smooth surfaces. In geophysics for example, we typically have different layers with very different physical properties. A very important question would be to know how our analysis would adapt to this situation. In fact, it seems natural to believe that sparsity would continue to hold in this more general setting. Intuitively, the wave group would still be approximated by rigid motion along the Hamiltonian flow. Only, one would need to account for possible reflections/refractions. A curvelet hitting a singularity at a small angle of incidence would typically produce two curvelets, a reflected and a refracted curvelet. This is merely an intuition which one would need to justify by a careful analysis quantifying the behavior of

a curvelet near the interface (here, the singular surface). We regard this type of question as an important extension to this work. 
The estimates proven in this paper are motivated by efforts towards applications as sparser expansions theoretically lead to better and faster algorithms. Our goal is to transform our theoretical insights into effective algorithms, and derive fast accurate solvers to certain classes of partial differential equations for which our methods have a comparative advantage. In this direction, suppose that a discretized version $E_{N}(t)$ of the curvelet matrix $E(t)$ is known in the sense that it has been precomputed once for all. Assuming that discretized version inherits the sparsity from its continuous analog, then for each initial value problem, we would have available a fast algorithm for calculating the solution of the full wave equation. Indeed, Corollary 1.2 asserts that the truncated matrix $\operatorname{trunc}\left(E_{N}(t)\right)$ obtained by keeping only $O\left(\epsilon^{1 / m}\right)$ terms per row obeys

$$
\left\|E_{N}(t)-\operatorname{trunc}\left(E_{N}(t)\right)\right\| \leq \epsilon .
$$

Therefore, ignoring the cost of the digital curvelet transform, the total cost of an algorithm calculating the solution of a full wave equation to within accuracy $\epsilon$ would be linear in the number of unknowns (number of voxels) and scale at most like $C(\epsilon) \cdot N$. This is not the most practically relevant situation, however, as in general, one would not know $E_{N}(t)$. Work in progress shows that one can still design effective algorithms to 'build' the matrix $E_{N}(t)$, and for all practical purposes compute the solution of the full wave equation for a given accuracy $\epsilon$ in $O\left(N(\log N)^{2}\right)$.

Finally, we would like to conclude by pointing out that there is now considerable evidence that the curvelet transform is a very useful mathematical architecture; curvelets can do things that other classical systems simply cannot do. This paper proved that tight-frames of curvelets provide optimally sparse representations of large classes of linear systems of hyperbolic differential equations. But they also allow for optimally sparse representations of wavefront phenomena [5]. Further, they can also be useful in many different settings. For example, they have useful microlocal features which make them especially suitable for deployment in many inverse problems and especially limited-angle tomography [8]. In short, curvelets addresses a new range of problems, going beyond what traditional multiscale systems offer.

\section{Appendix}

\subsection{Additional proofs for section 2}

Proof of proposition 2.2

These four properties were already formulated in [27], although with a slightly weaker definition of pseudo-distance. Properties 1 and 2 are not proved in that reference, and property 3 is not extensively documented. We give the justification for these three results for completeness.

1. We are to show that $d\left(\mu, \mu^{\prime}\right) \asymp d\left(\mu^{\prime}, \mu\right)$. With $e_{\mu}=\xi_{\mu} /\left|\xi_{\mu}\right|$, this is

$$
\left|\left\langle e_{\mu}, \Delta x\right\rangle\right|+|\Delta x|^{2}+|\Delta \theta|^{2} \asymp\left|\left\langle e_{\mu^{\prime}}, \Delta x\right\rangle\right|+|\Delta x|^{2}+|\Delta \theta|^{2} .
$$

It is sufficient to notice that

$$
\left|\left\langle e_{\mu}, \Delta x\right\rangle\right|+|\Delta x|^{2}+|\Delta \theta|^{2} \asymp\left|\left\langle e_{\mu}, \Delta x\right\rangle\right|+\left|\left\langle e_{\mu^{\prime}}, \Delta x\right\rangle\right|+|\Delta x|^{2}+|\Delta \theta|^{2} .
$$




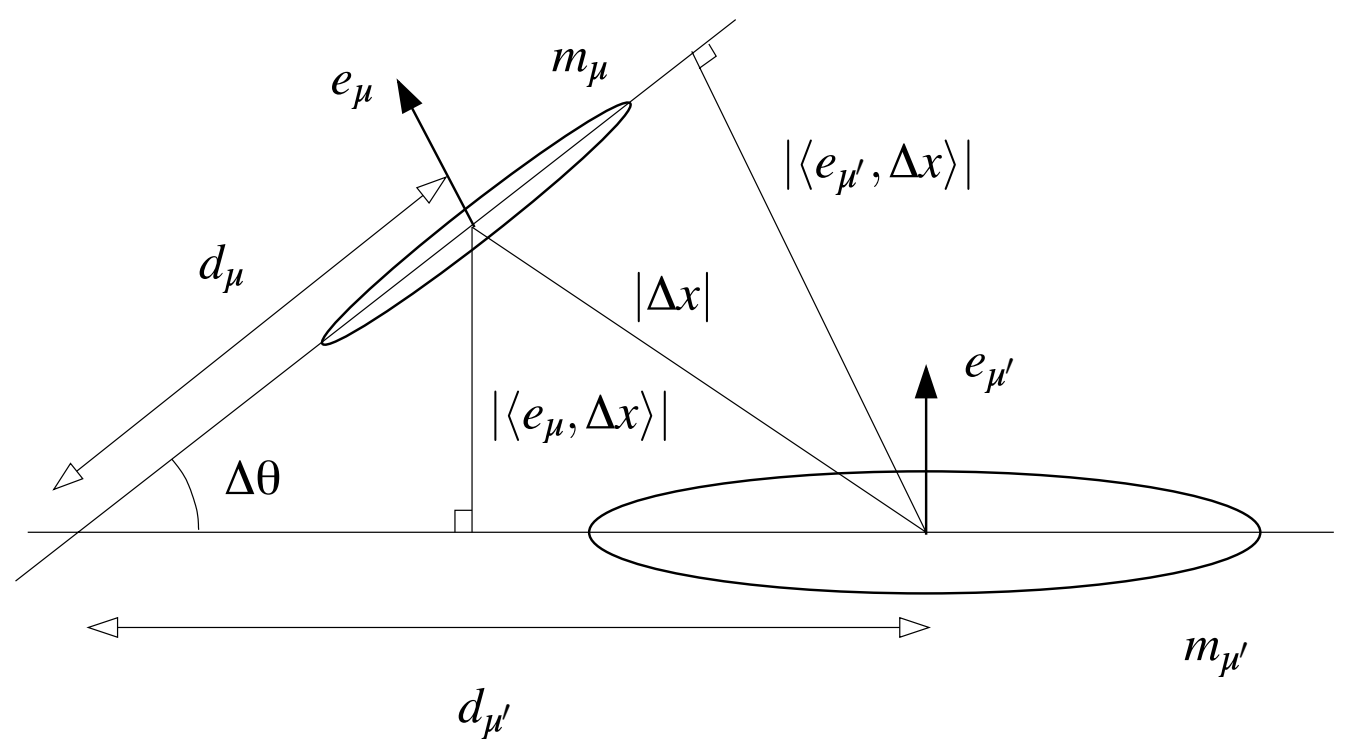

Figure 6: Relative position and orientation of two curvelet molecules in $x$-space. The ellipses indicate their essential support.

In order to justify the nontrivial inequality, use the law of cosines illustrated in Figure 6 :

$$
\begin{aligned}
\left|\left\langle e_{\mu}, \Delta x\right\rangle\right|^{2}+\left|\left\langle e_{\mu^{\prime}}, \Delta x\right\rangle\right|^{2} & =\sin ^{2}|\Delta \theta|\left(d_{\mu}^{2}+d_{\mu^{\prime}}^{2}\right) \\
& =\sin ^{2}|\Delta \theta||\Delta x|^{2} \pm 2\left|\left\langle e_{\mu}, \Delta x\right\rangle\right|\left|\left\langle e_{\mu^{\prime}}, \Delta x\right\rangle\right| \cos |\Delta \theta| \\
& \leq \sin ^{2}|\Delta \theta||\Delta x|^{2}+2\left|\left\langle e_{\mu}, \Delta x\right\rangle\right|\left|\left\langle e_{\mu^{\prime}}, \Delta x\right\rangle\right| .
\end{aligned}
$$

It follows that ||$\left\langle e_{\mu}, \Delta x\right\rangle|-|\left\langle e_{\mu^{\prime}}, \Delta x\right\rangle|| \leq C \cdot|\Delta \theta||\Delta x| \leq C \cdot\left(|\Delta \theta|^{2}+|\Delta x|^{2}\right)$ and, therefore,

$$
\left|\left\langle e_{\mu}, \Delta x\right\rangle\right|+\left|\left\langle e_{\mu^{\prime}}, \Delta x\right\rangle\right| \leq C \cdot\left(2\left|\left\langle e_{\mu}, \Delta x\right\rangle\right|+|\Delta \theta|^{2}+|\Delta x|^{2}\right) .
$$

2. Recall that $\omega\left(\mu, \mu^{\prime}\right)=2^{\left|j-j^{\prime}\right|}\left(1+2^{\min \left(j, j^{\prime}\right)} d\left(\mu, \mu^{\prime}\right)\right)$. Let us show that $d\left(\mu, \mu^{\prime}\right) \leq C$. $\left(d\left(\mu, \mu^{\prime \prime}\right)+d\left(\mu^{\prime \prime}, \mu^{\prime}\right)\right)$. To simplify notations, set in the coordinates defined by $\left\{e_{\mu}, e_{\mu}^{\perp}\right\}$,

$$
\begin{array}{lcc}
x_{\mu}=(0,0) & x_{\mu^{\prime}}=\left(x_{1}, x_{2}\right) & x_{\mu^{\prime \prime}}=\left(y_{1}, y_{2}\right) \\
e_{\mu}=(1,0) & e_{\mu^{\prime}}=(\cos \alpha, \sin \alpha) & e_{\mu^{\prime \prime}}=(\cos \beta, \sin \beta) \\
\left|\theta_{l}-\theta_{l^{\prime \prime}}\right|=|\beta| & \left|\theta_{l^{\prime}}-\theta_{l^{\prime \prime}}\right|=|\alpha-\beta|
\end{array}
$$

It is enough to show that there exists $\epsilon>0$ such that

$\epsilon\left|x_{1}\right| \leq\left|y_{1}\right|+\left|\cos \alpha\left(x_{1}-y_{1}\right)+\sin \beta\left(x_{2}-y_{2}\right)\right|+(|\beta|+|\alpha-\beta|)\left(\left|y_{1}\right|+\left|x_{1}-y_{1}\right|+\left|y_{2}\right|+\left|x_{2}-y_{2}\right|\right)$, because then $(|\beta|+|\alpha-\beta|)\left(\left|y_{1}\right|+\left|x_{1}-y_{1}\right|+\left|y_{2}\right|+\left|x_{2}-y_{2}\right|\right) \leq C \cdot\left(|\beta|^{2}+|\alpha-\beta|^{2}+\right.$ $\left.\left|y_{1}\right|^{2}+\left|x_{1}-y_{1}\right|^{2}+\left|y_{2}\right|^{2}+\left|x_{2}-y_{2}\right|^{2}\right)$. By contradiction let us assume that the inequality fails. Then we must have $\left|y_{1}\right|<\epsilon\left|x_{1}\right|$. It is always true that $\left|x_{1}-y_{1}\right|+\left|y_{1}\right| \geq\left|x_{1}\right|$ 
so it is necessary that $|\beta|+|\alpha-\beta|<\epsilon$. But then $|\alpha|<2 \epsilon$ thus $\cos \alpha>1-4 \epsilon^{2}$ and $|\sin \alpha|<2 \epsilon$. The term $\left|\cos \alpha\left(x_{1}-y_{1}\right)+\sin \beta\left(x_{2}-y_{2}\right)\right|$ is therefore always greater than $\left(1-4 \epsilon^{2}\right)\left|x_{1}-y_{1}\right|-\epsilon\left|x_{2}-y_{2}\right|$. But this quantity must also be less than $\epsilon\left|x_{1}-y_{1}\right|$, otherwise its sum with $\left|y_{1}\right|$ would exceed $\epsilon\left|x_{1}\right|$. So we must have $\left|x_{2}-y_{2}\right|>\frac{1-\epsilon-4 \epsilon^{2}}{\epsilon}\left|x_{1}-y_{1}\right|$. But then the sum $\left|y_{1}\right|+\left|x_{1}-y_{1}\right|+\left|x_{2}-y_{2}\right|$ must dominate $\frac{\left|x_{1}\right|}{2 \epsilon}$, which implies $|\beta|+|\alpha-\beta| \leq 2 \epsilon^{2}$. By induction, $\alpha=\beta=0$ and $\left|y_{1}\right|+\left|x_{1}-y_{1}\right| \geq\left|x_{1}\right|$ yields a contradiction.

3. Let us show that $\sum_{\mu_{1}} \omega\left(\mu_{0}, \mu_{1}\right)^{-N} \cdot \omega\left(\mu_{1}, \mu_{2}\right)^{-N} \leq C_{N} \cdot \omega\left(\mu_{0}, \mu_{2}\right)^{-(N-1)}$. We closely follow and expand the argument in [27]. We will need to use $d\left(\mu_{0}, \mu_{1}\right) \asymp d\left(\mu_{1}, \mu_{0}\right)$, as we have just showed.

Define $I_{\mu_{1}}$ by

$$
\begin{aligned}
I_{\mu_{1}} & :=\omega\left(\mu_{2}, \mu_{1}\right)^{-N} \cdot \omega\left(\mu_{1}, \mu_{0}\right)^{-N} \\
& =\left(2^{\left|j_{2}-j_{1}\right|+\left|j_{1}-j_{0}\right|}\left(1+2^{\min \left(j_{2}, j_{1}\right)} d\left(\mu_{2}, \mu_{1}\right)\right)\left(1+2^{\min \left(j_{0}, j_{1}\right)} d\left(\mu_{0}, \mu_{1}\right)\right)\right)^{-N} .
\end{aligned}
$$

To ease notations, put temporarily $a_{0}=2^{\min \left(j_{0}, j_{1}\right)}, a_{2}=2^{\min \left(j_{2}, j_{1}\right)}, d_{01}=d\left(\mu_{0}, \mu_{1}\right)$, and $d_{12}=d\left(\mu_{2}, \mu_{1}\right)$. We develop a lower bound on $\left(1+a_{2} d_{12}\right)\left(1+a_{0} d_{01}\right)=1+a_{2} d_{12}+$ $a_{0} d_{01}+a_{2} d_{12} a_{0} d_{01}$. We make three simple observations: first,

$$
a_{2} d_{12}+a_{0} d_{01} \geq \min \left(a_{2}, a_{0}\right)\left(d_{12}+d_{01}\right)=A_{0}, \quad \text { and } \quad d_{12}+d_{01} \geq C \cdot d\left(\mu_{0}, \mu_{2}\right) ;
$$

second,

$$
a_{2} d_{12}+a_{0} d_{01} \geq \max \left(a_{2} d_{12}, a_{0} d_{01}\right) \geq \max \left(a_{2}, a_{0}\right) \min \left(d_{12}, d_{01}\right)=B_{0} ;
$$

and third

$$
\begin{aligned}
a_{2} d_{12} a_{0} d_{01} & =\max \left(a_{2}, a_{0}\right) \min \left(a_{2}, a_{0}\right) \max \left(d_{12}, d_{01}\right) \min \left(d_{12}, d_{01}\right) \\
& \geq \max \left(a_{2}, a_{0}\right) \min \left(a_{2}, a_{0}\right) \min \left(d_{12}, d_{01}\right) \frac{d_{12}+d_{01}}{2}=A_{0} B_{0} / 2
\end{aligned}
$$

This gives

$$
1+a_{2} d_{12}+a_{0} d_{01}+a_{2} d_{12} a_{0} d_{01} \geq \frac{1}{2}\left(1+A_{0}+B_{0}+A_{0} B_{0}\right) \geq \frac{1}{2}\left(1+A_{0}\right)\left(1+B_{0}\right) .
$$

We replace the values of $A_{0}, B_{0}$ by their expression, use the relation $A_{0} \geq d\left(\mu_{0}, \mu_{2}\right)$ and obtain

$$
I_{\mu_{1}} \leq C \cdot 2^{-\left(\left|j_{2}-j_{1}\right|+\left|j_{0}-j_{1}\right|\right) N} \cdot\left(1+2^{\min \left(j_{2}, j_{0}, j_{1}\right)} d\left(\mu_{2}, \mu_{0}\right)\right)^{-N} \cdot\left(L_{1}\right)^{-N}
$$

with

$$
L_{1}=1+\max \left(2^{\min \left(j_{2}, j_{1}\right)}, 2^{\min \left(j_{0}, j_{1}\right)}\right) \min \left(d_{01}, d_{12}\right) .
$$

Note that

$$
\begin{aligned}
L_{1} & =\min \left(1+\max \left(2^{\min \left(j_{2}, j_{1}\right)}, 2^{\min \left(j_{0}, j_{1}\right)}\right) d_{12}, 1+\max \left(2^{\min \left(j_{2}, j_{1}\right)}, 2^{\min \left(j_{0}, j_{1}\right)}\right) d_{01}\right) \\
& \geq \min \left(1+2^{\min \left(j_{2}, j_{1}\right)} d_{12}, 1+2^{\min \left(j_{0}, j_{1}\right)} d_{01}\right)
\end{aligned}
$$


and, therefore,

$$
\begin{aligned}
\left(L_{1}\right)^{-N} & \leq \max \left(\left(1+2^{\min \left(j_{2}, j_{1}\right)} d_{12}\right)^{-N},\left(1+2^{\min \left(j_{0}, j_{1}\right)} d_{01}\right)^{-N}\right) \\
& \leq\left(1+2^{\min \left(j_{2}, j_{1}\right)} d_{12}\right)^{-N}+\left(1+2^{\min \left(j_{0}, j_{1}\right)} d_{01}\right)^{-N}
\end{aligned}
$$

In the sequel we will repeatedly make use of the bound

$$
\sum_{k, \ell}\left(1+2^{q} d\left(\mu, \mu^{\prime}\right)\right)^{-N} \leq C \cdot 2^{2(j-q)_{+}},
$$

valid for $N \geq 2$, any real $q$ and where the subscript + denotes the positive part. This is justified as follows. Without loss of generality, assume that $\mu^{\prime}=\left(j^{\prime}, 0,0\right)$ so that the curvelet $\gamma_{\mu^{\prime}}$ is nearly vertical and centered near the origin. We recall that $\Delta \theta=\pi \cdot \ell \cdot 2^{-\lfloor j / 2\rfloor}, \ell=0,1, \ldots 2^{\lfloor j / 2\rfloor}-1$, and $x_{\mu}=R_{\theta_{\mu}} D_{j}^{-1} k$, say. Then the left-hand side is

$$
\sum_{\ell=0}^{2^{\lfloor j / 2\rfloor}-1} \sum_{k \in \mathbb{Z}^{2}}\left(1+2^{q}\left(\left|2^{-j / 2} \ell\right|^{2}+\left|2^{-j / 2} k_{2}\right|^{2}+\left|2^{-j} k_{1}\right|\right)\right)^{-N} .
$$

For $j \geq q$ this can be seen as a Riemann sum and bounded-up to a numerical multiplicative constant - by the corresponding integral

$$
\int_{\mathbb{R}^{2}} \frac{d x}{2^{-3 j / 2}} \int_{\mathbb{R}} \frac{d y}{2^{-j / 2}}\left[1+2^{q}\left(y^{2}+x_{2}^{2}+\left|x_{1}\right|\right)\right]^{-N}
$$

which in turn is less than $C \cdot 2^{2(j-q)}$ provided $N \geq 2$. For $j \leq q$, the sum (7.4) essentially consists of a few terms, giving a $O(1)$ contribution. This gives the bound $C \cdot 2^{2(j-q)_{+}}$.

By symmetry, we can now assume $j_{0} \leq j_{2}$. Let us consider three cases.

- $\mathbf{0} \leq \mathbf{j}_{2} \leq \mathbf{j}_{1}$. In that case we have the bound

$$
\left(L_{1}\right)^{-N} \leq C \cdot\left[\left(1+2^{j_{2}} d_{01}\right)^{-N}+\left(1+2^{j_{2}} d_{12}\right)^{-N}\right] .
$$

Summing this quantity over $k_{1}$ and $\ell_{1}$ i.e., over all $\mu_{1}$ that correspond to a given $j_{1}$, and using (7.3), we obtain for $j_{1} \geq j_{2}$

$$
\begin{aligned}
\sum_{\mu_{1}} I_{\mu_{1}} & \leq C \cdot\left(1+2^{j_{0}} d_{02}\right)^{-N} \sum_{j_{1} \geq j_{2}} 2^{-\left(2 j_{1}-j_{0}-j_{2}\right) N} \cdot 2^{2\left(j_{1}-j_{2}\right)} \\
& \leq C \cdot 2^{-\left(j_{2}-j_{0}\right) N}\left(1+2^{j_{0}} d_{02}\right)^{-N}=C \cdot \omega\left(\mu_{0}, \mu_{2}\right)^{-N} .
\end{aligned}
$$

- $\mathbf{0} \leq \mathbf{j}_{\mathbf{1}} \leq \mathbf{j}_{\mathbf{0}}$. We now have

$$
\left(L_{1}\right)^{-N} \leq C \cdot\left[\left(1+2^{j_{1}} d_{01}\right)^{-N}+\left(1+2^{j_{1}} d_{12}\right)^{-N}\right] .
$$

According to (7.3), the sum over $k_{1}$ and $\ell_{1}$ of $\left(L_{1}\right)^{-N}$ is bounded by a constant independent of $j_{1}$. The remaining sum is

$$
\sum_{\mu_{1}} I_{\mu_{1}} \leq C \cdot 2^{-\left(j_{0}+j_{2}\right) N} \sum_{j_{1} \leq j_{0}} 2^{2 j_{1} N} \cdot\left(1+2^{j_{1}} d_{02}\right)^{-N}
$$

Observe that $2^{j_{1} N}\left(1+2^{j_{1}} d_{02}\right)^{-N} \leq 2^{j_{0} N}\left(1+2^{j_{0}} d_{02}\right)^{-N}$, therefore

$$
\sum_{\mu_{1}} I_{\mu_{1}} \leq C \cdot 2^{-\left(j_{2}-j_{0}\right) N}\left(1+2^{j_{0}} d_{02}\right)^{-N}=C \cdot \omega\left(\mu_{0}, \mu_{2}\right)^{-N} .
$$


- $\mathbf{j}_{\mathbf{0}} \leq \mathbf{j}_{\mathbf{1}} \leq \mathbf{j}_{\mathbf{2}}$. In that case we still have

$$
\left(L_{1}\right)^{-N} \leq C \cdot\left[\left(1+2^{j_{1}} d_{01}\right)^{-N}+\left(1+2^{j_{1}} d_{12}\right)^{-N}\right] .
$$

summed over $k_{1}$ and $\ell_{1}$ into a $O(1)$ contribution. What remains is

$$
\begin{array}{r}
\sum_{\mu_{1}} I_{\mu_{1}} \leq C \cdot 2^{-\left(j_{2}-j_{0}\right) N}\left(1+2^{j_{0}} d_{02}\right)^{-N} \sum_{j_{0} \leq j_{1} \leq j_{2}} 1 \\
\leq C \cdot \omega\left(\mu_{0}, \mu_{2}\right)^{-(N-1)} .
\end{array}
$$

We conclude by collecting the estimates corresponding to the three different cases. Remark that the loss of one (fractional) power of $\omega$ in the third case is unavoidable unless one modifies its definition in the spirit of [27]. This would however make notations unnecessarily heavy.

4. See [27] p. 804.

Proof of the inequality (2.14). Assume without loss of generality that $\mu=\mu_{0}$. We may express $S_{\mu^{\prime}}(\xi)$ as $S_{\mu_{0}^{\prime}}\left(R_{\Delta \theta} \xi\right)$, with $\Delta \theta=\theta_{\mu}-\theta_{\mu^{\prime}}$. We begin by expressing the integral in polar coordinates,

$$
\begin{array}{ll}
\xi_{1}=r \cos \theta & \left(R_{\Delta \theta} \xi\right)_{1}=r \cos (\theta+\Delta \theta), \\
\xi_{2}=r \sin \theta & \left(R_{\Delta \theta} \xi\right)_{2}=r \sin (\theta+\Delta \theta) .
\end{array}
$$

As we can see, the cosine factor is not crucial and we may just as well drop it. Consequently,

$$
\begin{aligned}
\int\left|S_{\mu}(\xi) S_{\mu^{\prime}}(\xi)\right|^{n} d \xi \leq & C \cdot \int_{0}^{\infty} r d r \frac{1}{\left[1+2^{-j} r\right]^{N}} \frac{1}{\left[1+2^{-j^{\prime}} r\right]^{N}} \\
& \times \int_{0}^{2 \pi} d \theta[1+a|\sin \theta|]^{-N}\left[1+a^{\prime}|\sin (\theta+\Delta \theta)|\right]^{-N}
\end{aligned}
$$

where $a=\frac{2^{-j / 2} r}{1+2^{-j} r}$ and $a^{\prime}=\frac{2^{-j^{\prime} / 2} r}{1+2^{-j^{\prime}} r}$. This decoupling makes the problem of bounding the inner integral on the variable $\theta$ tractable. For example when $a>a^{\prime}>1$, following [23] p.56,

$$
\int_{-\infty}^{\infty} d \theta[1+a|\theta|]^{-N}\left[1+a^{\prime}|\theta+\Delta \theta|\right]^{-N} \leq C \cdot \frac{1}{a} \frac{1}{\left[1+a^{\prime}|\Delta \theta|\right]^{N}}
$$

We get other estimates for other values and orderings of $a$ and $a^{\prime}$. The integral on $r$ is then broken up into several pieces according to the values of $a, a^{\prime}, j$ and $j^{\prime}$. It is straightforward to show that each of these contributions satisfies the inequality (2.14).

\subsection{Additional proofs for section 5}

Proof of lemma 5.1

By definition $a^{(\mu)}(x)=2^{-3 j / 4} m_{\mu}\left(D_{2^{-j}} R_{\theta_{\mu}} x-k\right)$ and, therefore,

$$
\begin{aligned}
a^{(\mu)}(x) & =\frac{1}{\alpha_{\mu}} \int(R f)(a, \theta, b) a^{3 / 4} 2^{-3 j / 4} \psi\left(D_{a} R_{\theta}\left(R_{\theta_{\mu}}^{-1} D_{2^{j}}(x+k)-b\right) d \mu\right. \\
& =\frac{1}{\alpha_{\mu}} \int(R f)(a, \theta, b)|A|^{1 / 2} \psi(A(x-(\beta-k)) d \mu
\end{aligned}
$$


where $A=D_{a} R_{\delta} D_{2^{j}}$ with $\delta=\theta-\theta_{\mu}$ and $\beta=D_{2^{-}{ }_{j}} R_{\theta_{\mu}} b$.

Let us first verify the assertion about the support of $a^{(\mu)}$. Recall that over a cell $Q_{\mu}$, $\beta \in\left[k_{1}, k_{1}+1\right) \times\left[k_{2}, k_{2}+1\right)$, and hence for all $b \in Q_{\mu}$, we have

$$
\operatorname{Supp} \psi(A(x-(\beta-k))) \subset \operatorname{Supp} \psi(A x)+[0,1]^{2} .
$$

Next $\operatorname{Supp} \psi(A x) \subset A^{-1}[0,1]^{2}$ with $A^{-1}=D_{2^{-j}} R_{-\delta} D_{a}^{-1}$. It is not difficult to check that $A^{-1}[0,1]^{2} \subset\left[c_{1}, c_{2}\right) \times\left[d_{1}, d_{2}\right)$ which then gives (5.15).

There are several ways to prove the property about nearly vanishing moments. A possibility is to show that the Fourier transform of $a^{(\mu)}$ is appropriately small in a neighborhood of the axis $\xi_{1}=0$. We choose a more direct strategy and show that

$$
\left|\int \psi(A(x-\beta)) x_{1}^{k} d x_{1}\right| \leq C_{m} \cdot 2^{-j(m+1)}
$$

uniformly over the $(a, \theta, b) \in Q_{\mu}$. The property (5.16) follows from this fact. Indeed,

$$
\int a^{(\mu)}\left(x_{1}, x_{2}\right) x_{1}^{k} d x_{1}=\frac{1}{\alpha_{\mu}} \int_{Q_{\mu}} R f(a, \theta, b) d \mu \int|A|^{1 / 2} \psi(A(x-\beta)) x_{1}^{k} d x_{1},
$$

and the Cauchy-Schwarz inequality gives

$$
\begin{aligned}
\left|\int a^{(\mu)}\left(x_{1}, x_{2}\right) x_{1}^{k} d x_{1}\right| & \leq \frac{1}{\alpha_{\mu}} \cdot\|R f\|_{L_{2}\left(Q_{\mu}\right)} \cdot\left(\left.\left.\int_{Q_{\mu}}\left|\int\right| A\right|^{1 / 2} \psi(A(x-\beta)) x_{1}^{k} d x_{1}\right|^{2} d \mu\right)^{1 / 2} \\
& =\left(\left.\left.\int_{Q_{\mu}}\left|\int\right| A\right|^{1 / 2} \psi(A(x-\beta)) x_{1}^{k} d x_{1}\right|^{2} d \mu\right)^{1 / 2} .
\end{aligned}
$$

The uniform bound (7.6) together with the fact that $\int_{Q_{\mu}} d \mu$ is either $3 \pi$ or $3 \pi / 2$ gives (5.16).

We then need to establish (7.6). Let $\partial_{2}$ be $\partial / \partial x_{2}$, recall that by assumption (5.8)-(5.9), we have that for all $x_{2} \in \mathbb{R}$,

$$
\int \partial_{2}^{n} \psi\left(x_{1}, x_{2}\right) x_{1}^{k} d x_{1}=0, \quad k=0,1, \ldots, R,
$$

and more generally, for each $\alpha \neq 0$ and $\beta$

$$
\int \partial_{2}^{n} \psi\left(\alpha x_{1}+\beta, x_{2}\right) x_{1}^{k} d x_{1}=0, \quad k=0,1, \ldots, R .
$$

We shall use (7.7) to prove (7.6). Letting

$$
A=\left(\begin{array}{ll}
a_{11} & a_{12} \\
a_{21} & a_{22}
\end{array}\right)
$$

and with the same notations as before, a simple calculation shows that $a_{21}=-\frac{2^{-j} \sin \delta}{\sqrt{a}}$. As $a \geq 2^{-(j+1)}$ and $|\delta| \leq \pi / 2 \cdot 2^{-\lfloor j / 2\rfloor}$, we have

$$
\left|a_{21}\right| \leq c \cdot 2^{-j}
$$


We then write

$$
\begin{aligned}
\psi(A x) & =\psi\left(a_{11} x_{1}+a_{12} x_{2}, a_{21} x_{1}+a_{22} x_{2}\right) \\
& =\sum_{n=0}^{N-1} D^{n} \psi\left(a_{11} x_{1}+a_{12} x_{2}, a_{22} x_{2}\right) \frac{\left(a_{21} x_{1}\right)^{n}}{n !}+O\left(\left(a_{21} x_{1}\right)^{N}\right)
\end{aligned}
$$

and, therefore,

$$
\int \psi(A x) x_{1}^{k} d x_{1}=\sum_{n=0}^{N-1} \frac{a_{21}^{n}}{n !} \int D^{n} \psi\left(a_{11} x_{1}+a_{12} x_{2}, a_{22} x_{2}\right) x_{1}^{n+k} d x_{1}+O\left(a_{21}^{N}\right)
$$

Fix $k \leq D$ and pick $N=D-k+1$ so that for $n=0,1, \ldots, N-1, n+k \leq D$. By virtue of (7.7) all the integrals in the sum vanish and the only remaining term is $O\left(a_{21}^{N}\right)$ which because of (7.8) is $O\left(2^{-j N}\right)$. As a consequence, setting $m=D / 2$, we conclude that

$$
\left|\int \psi(A x) x_{1}^{k} d x_{1}\right| \leq C_{m} \cdot 2^{-j(m+1)}, \quad k=0,1, \ldots m
$$

this is the content of (7.6).

The careful reader will notice that inequality (7.6) or equivalently (5.16) is a weaker statement than inequality (2.6) for the definition of nearly vanishing moments. There is no doubt that the stronger estimate (2.6) also holds for curvelet atoms. The proof of this fact uses standard arguments and we choose not to reproduce it here.

Last, the regularity property is a simple consequence of the Cauchy Schwarz inequality;

$$
\begin{aligned}
\left|a^{(\mu)}\left(x_{1}, x_{2}\right)\right| & \leq \frac{1}{\alpha_{\mu}} \int\left|R f(a, \theta, b)\left\|\left.A\right|^{1 / 2}\right\| \psi \|_{L_{\infty}} d \mu\right. \\
& \leq\|\psi\|_{L_{\infty}} \cdot \frac{1}{\alpha_{\mu}}\|R f\|_{L_{2}\left(Q_{\mu}\right)} \cdot\left(\int_{Q_{\mu}}|A| d \mu\right)^{1 / 2} \\
& =2 \sqrt{3 \pi} \cdot\|\psi\|_{L_{\infty}} .
\end{aligned}
$$

These last inequalities used the facts that $|A| \leq 4$ for $(a, \theta, b) \in Q_{\mu}$ and $\int_{Q_{\mu}} d \mu \leq 3 \pi$. Estimates for higher derivatives are obtained in exactly the same fashion-after differentiation of the integrand. This finishes the proof of the lemma.

Proof of (5.18). Recall that

$$
\alpha_{\mu \mu^{\prime}}=\left(\int_{Q_{\mu^{\prime}}}\left|\mathcal{R}\left(q(D) \gamma_{\mu}\right)(a, b, \theta)\right|^{2} d \mu\right)^{1 / 2} .
$$

The first thing to notice is that $q(D) \gamma_{\mu}$ is still a family of curvelet molecules, because $q(\xi)$ is a multiplier of order zero. Since $\psi_{a, \theta, b}$ also obeys the molecule properties, lemma 2.3 implies the corresponding almost-orthogonality condition. Integrating over $Q_{\mu^{\prime}}$ does not compromise this estimate, as can be seen by applying the Cauchy-Schwarz inequality.

Proof of inequality (5.2). Derivatives of $\hat{\gamma}_{\mu}$ and $\sigma$ are treated using the following estimates.

$$
\begin{aligned}
& \left|\partial_{\xi}^{\alpha} \hat{\gamma}_{\mu}(\xi)\right| \leq C_{\alpha} \cdot 2^{-3 j / 4} 2^{-\alpha_{1} j} 2^{-\alpha_{2} j / 2} \\
& \left|\partial_{\xi}^{\alpha} \sigma\left(\phi^{-1}(x), \xi\right)\right| \leq C_{\alpha} \cdot 2^{-|\alpha| j} \quad \text { on } W_{\mu}=\operatorname{supp}\left(\hat{\gamma}_{\mu}\right) .
\end{aligned}
$$


We now develop size estimates for the phase perturbation $\delta$. Following closely the discussion in [29], p.407, we claim that on $W_{\mu}$,

$$
\left|\partial_{\xi}^{\alpha} \partial_{x}^{\beta} \delta(x, \xi)\right| \leq C_{\alpha \beta} \cdot 2^{-\alpha_{1} j} 2^{-\alpha_{2} j / 2} .
$$

The derivations in $x$ add no complications. Hence, assume that $\beta=0$. As the above result (7.9) relies upon the homogeneity of the phase with respect to $\xi$, we recall a few useful facts about homogeneous functions of degree one:

$$
\begin{aligned}
& \Phi=\Phi_{\xi} \cdot \xi \quad \text { (Euler's theorem), } \\
& \Phi_{\xi \xi} \cdot \xi=0 \quad \text { (differentiate the above relation), } \\
& \partial_{\xi}^{\alpha} \Phi=O\left(|\xi|^{1-|\alpha|}\right) .
\end{aligned}
$$

It follows from the definition that $\delta\left(x, \xi_{1}, 0\right)=0$ and likewise $\frac{\partial \delta}{\partial \xi_{2}}\left(x, \xi_{1}, 0\right)=0$. Thus for every $n, \frac{\partial^{n} \delta}{\partial \xi_{1}^{n}}\left(x, \xi_{1}, 0\right)=0$ and $\frac{\partial}{\partial \xi_{2}} \frac{\partial^{n} \delta}{\partial \xi_{1}^{n}}\left(x, \xi_{1}, 0\right)=0$. Recall that the support conditions are $\left|\xi_{1}\right| \leq C \cdot 2^{j}$ and $\left|\xi_{2}\right| \leq C \cdot 2^{j / 2}$. Taylor series expansions about $\xi_{2}=0$ together with homogeneity assumptions give

$$
\begin{aligned}
\frac{\partial^{\alpha_{1}}}{\partial \xi_{1}^{\alpha_{1}}} \delta(x, \xi) & =O\left(\left|\xi_{2}\right|^{2}|\xi|^{-1-\alpha_{1}}\right)=O\left(2^{-\alpha_{1} j}\right), \\
\frac{\partial}{\partial \xi_{2}} \frac{\partial^{\alpha_{1}}}{\partial \xi_{1}^{\alpha_{1}}} \delta(x, \xi) & =O\left(\left|\xi_{2}\right||\xi|^{-1-\alpha_{1}}\right)=O\left(2^{-j / 2} 2^{-\alpha_{1} j}\right), \\
\frac{\partial^{\alpha_{2}}}{\partial \xi_{2}^{\alpha_{2}}} \frac{\partial^{\alpha_{1}}}{\partial \xi_{1}^{\alpha_{1}}} \delta(x, \xi) & =O\left(|\xi|^{1-\alpha_{1}-\alpha_{2}}\right)=O\left(2^{-\alpha_{1} j} 2^{-\alpha_{2} j / 2}\right) \quad \text { when } \alpha_{2} \geq 2,
\end{aligned}
$$

as claimed. The point about these estimates is that they exhibit exactly the parabolic scaling of curvelets. We conclude

$$
\left|\partial_{\xi}^{\alpha} e^{i \delta\left(\phi^{-1}(x), \xi\right)}\right| \leq C_{\alpha} \cdot 2^{-\alpha_{1} j} 2^{-\alpha_{2} j / 2} \quad \text { on } W_{\mu}
$$

and therefore (5.2).

\section{References}

[1] J.P. Antoine, R. Murenzi, Two-dimensional directional wavelets and the scale-angle representation, Sig. Process., 52 (1996) 259-281

[2] G. Beylkin, R. Coifman, V. Rokhlin, Fast wavelet transforms and numerical algorithms, Comm. on Pure and Appl. Math., 44 (1991), 141-183

[3] E. J. Candès, L. Demanet, Curvelets and Fourier Integral Operators, C. R. Acad. Sci. Paris, Ser. I, 336 (2003), 395-398

[4] E. J. Candès, D. L. Donoho, Curvelets - a suprisingly effective nonadaptive representation for objects with edges, in Curves and Surface Fitting : Saint-Malo 1999, A. Cohen, C. Rabut, L. Schumaker (eds.), Vanderbilt University Press, Nashville, 2000, $105-120$

[5] E. J. Candès, D. L. Donoho, New Tight Frames of Curvelets and Optimal Representations of Objects with Piecewise $C^{2}$ Singularities, Comm. on Pure and Appl. Math., 57 (2004), 219-266. 
[6] E. J. Candès, D. L. Donoho, Directional Wavelet Transform via Parabolic Scaling: I. Resolution of the Wavefront Set, Technical Report, Stanford University, 2003.

[7] E. J. Candès, D. L. Donoho, DCTvUSFFT: Digital Curvelet Transforms via Unequispaced Fast Fourier Transforms, Technical Report, California Institute of Technology, 2004 .

[8] E. J. Candès, D. L. Donoho, Curvelets: New Tools for Limited-Angle Tomography, Technical Report, California Institute of Technology, 2004.

[9] E. J. Candès, F. Guo, New multiscale transforms, minimum total variation synthesis : Application to edge-preserving image reconstruction, Sig. Process., special issue on Image and Video Coding Beyond Standards, 82 (2002), 1519-1543.

[10] A. Córdoba, C. Fefferman, Wave packets and Fourier integral operators, Comm. PDE's, 3(11) (1978), 979-1005

[11] D. L. Donoho, M. Vetterli, R. A. DeVore, I. Daubechies, Data compression and harmonic analysis, IEEE Trans. Inform. Theory, 44, (1998), 2435-2476

[12] D. L. Donoho, De-noising by Soft-Thresholding, IEEE Trans. Inform. Theory, 41, (1995), 613-627

[13] J. Duistermaat, Fourier Integral Operators, Birkhauser, Boston, 1996

[14] L. Garding, Singularities in Linear Wave Propagation, Lecture notes in Math 1241, Springer, 1987

[15] C. Fefferman, A note on spherical summation multipliers, Israel J. Math. 15 (1973) 44-52

[16] G. Folland, Harmonic Analysis in Phase Space, Princeton University Press, 1989

[17] M. Frazier, B. Jawerth, G. Weiss, Littlewood-Paley Theory and the Study of Function Spaces, CBMS 79, AMS, Providence, 1991

[18] P. Lax, Asymptotic solutions of oscillatory initial value problems, Duke Math J., 24 (1957) 627-646

[19] P. G. Lemarié and Y. Meyer, Ondelettes et bases Hilbertiennes. Rev. Mat. Iberoamericana, 2 (1986), 1-18.

[20] Y. Meyer. Wavelets: Algorithms and Applications, SIAM, Philadelphia, 1993.

[21] S. Mallat, A Wavelet Tour of Signal Processing, 2nd ed., Academic Press, Orlando-San Diego, 1999.

[22] Y. Meyer, Ondelettes et Opérateurs, Hermann, Paris, 1990

[23] Y. Meyer, R. Coifman, Wavelets, Calderón-Zygmund and Multilinear Operators, Cambridge Univresity Press, Cambridge, 1997

[24] A. Seeger, C. Sogge, E. Stein, Regularity properties of Fourier integral operators, Annals of Math. 134 (1991), 231-251 
[25] R. Seeley, Complex powers of an elliptic operator, Proc. Symp. Pure Math 10 (1968), 288-307

[26] H. Smith, A Hardy space for Fourier integral operators, J. Geom. Anal. 7 (1997)

[27] H. Smith, A parametrix construction for wave equations with $C^{1,1}$ coefficients, Ann. Inst. Fourier, Grenoble, 48, 3 (1998), 797-835

[28] C. Sogge, Fourier Integrals in Classical Analysis, Cambridge University Press, 1993

[29] E. Stein, Harmonic Analysis, Princeton University Press, Princeton NJ, 1993

[30] C. Stolk, M. de Hoop, Microlocal Analysis of Seismic Inverse Scattering in Anisotropic Elastic Media, Comm. Pure and Appl. Math., 55 (2002), 261-301

[31] M. Taylor, Reflection of Singularities of Solutions to Systems of Differential Equations, Comm. Pure and Appl. Math., 28 (1975), 457-478

[32] F. Trèves, Introduction to Pseudo-Differential and Fourier Integral Operators, Plenum press, 1982, 2 volumes.

[33] G. Whitham, Linear and Nonlinear waves, Wiley Interscience, 1999. 